\title{
On Stability of Generalized Short-Crested Water Waves
}

\author{
BY \\ TRAVIS R. MCBRIDE \\ B.A., DePaul University, 1990 \\ M.S., University of Illinois at Chicago, 1993
}

\section{THESIS}

Submitted in partial fulfillment of the requirements for the degree of Doctor of Philosophy in Mathematics in the Graduate College of the University of Illinois at Chicago, 2012

Chicago, Illinois

Defense Committee:

Alexey Cheskidov

David P. Nicholls, Chair and Advisor

Thomas J. Royston, Bioengineering

Roman Shvydkoy

Jan Verschelde 
Copyright by

Travis R. McBride

2012 
To my wife Angela,

and my daughters Linnea and Elsa,

for their love and support on this very long road. 


\section{ACKNOWLEDGMENTS}

I would first like to thank my advisor David Nicholls for his invaluable guidance and instruction over the course of my graduate studies. He has been both a guide and interpreter in the voluminous and sometimes daunting realm of water waves literature. I would also like to thank my remaining committee members, Alexey Cheskidov, Thomas Royston, Roman Shvydkoy, and Jan Verschelde. I gratefully acknowledge support from the Department of Energy as a graduate research assistant through Award No. DE-SC0001549, under which the majority of this work was done, and from MSCS as a teaching assistant.

Many other teachers over the years have had a significant influence on my intellectual development: Eugene Kirshner of Pierce Elementary in Chicago; the late Harold Brownstein of Senn High School in Chicago; J. Marshall Ash, Barbara Cortzen, and Constantine Georgakis of DePaul University; and Charles Knessl of MSCS at UIC.

Finally, I would like to thank my parents, Raymond and Marcie McBride, who instilled a love of learning in me from an early age, and my family and friends for providing anchor during the ups and downs of graduate school. 


\section{TABLE OF CONTENTS}

CHAPTER

PAGE

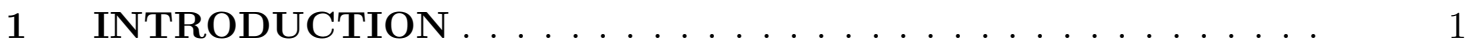

1.1 Established Results for Short-Crested Waves . . . . . . . . . 2

1.2 Our Study of Generalized Short-Crested Waves . . . . . . . . . 3

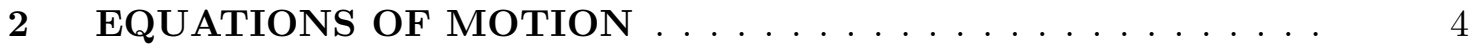

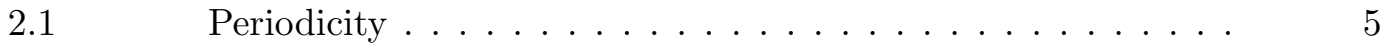

$2.2 \quad$ Surface Formulation $\ldots \ldots \ldots \ldots \ldots$

$2.3 \quad$ The First Variations of $A(\eta)$ and $B(\eta, \xi) \ldots \ldots \ldots . \ldots 10$

2.4 The Hamiltonian . . . . . . . . . . . . . . . . 16

2.5 Traveling Wave Evolution Equations . . . . . . . . . . . 17

$2.6 \quad$ Dimensionless Variables . . . . . . . . . . . . . . . . 18

$2.7 \quad$ Spectral Stability Analysis . . . . . . . . . . . . . . 27

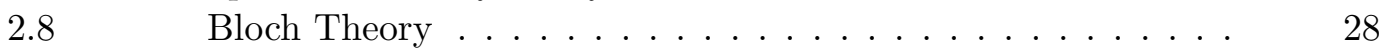

3 THE DIRICHLET-NEUMANN OPERATOR AND ITS FIRST

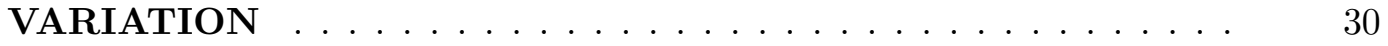

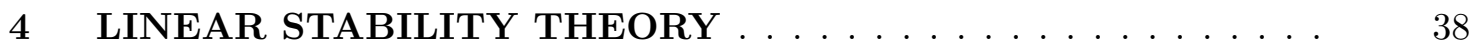

5 GENERALIZED SHORT CRESTED WAVES . . . . . . . . . . 42

6 NUMERICAL METHOD AND PARALLEL APPROACH . . . 47

$6.1 \quad$ Numerical Method . . . . . . . . . . . . . . . 47

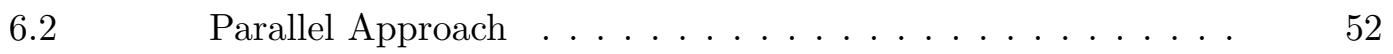

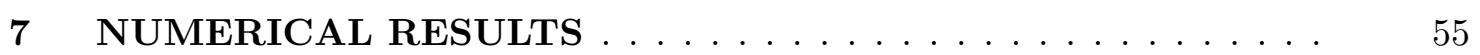

$7.1 \quad$ GSCW Surface and Contour Plots . . . . . . . . . 55

$7.2 \quad$ Numerical Simulations: Overview . . . . . . . . . . . . 63

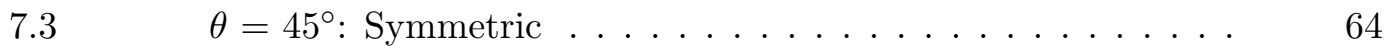

$7.4 \quad \theta=60^{\circ}$ : Moderately Skewed . . . . . . . . . . 73

$7.5 \quad \theta=75^{\circ}$ : Strongly Asymmmetric . . . . . . . . . 81

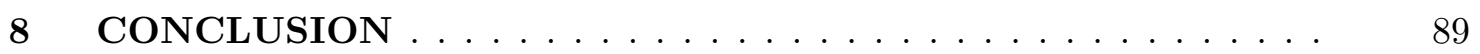

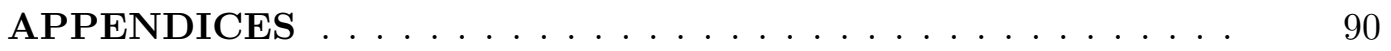

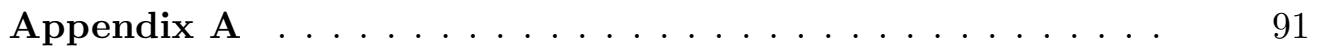


TABLE OF CONTENTS (Continued)

CHAPTER

$\underline{\text { PAGE }}$

CITED LITERATURE $\ldots \ldots \ldots \ldots \ldots \ldots$

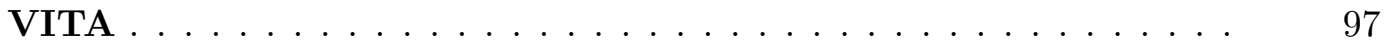




\section{LIST OF TABLES}

I COMMON VALUES ACROSS ALL SIMULATIONS . . . . . . . . 54

II $\quad$ ZONES OF INSTABILITY FOR $\theta=45^{\circ} \ldots \ldots \ldots \ldots$

III DOMINANT INSTABILITY FOR SOME CHOSEN VALUES OF $\alpha$ FOR $\theta=45^{\circ} \ldots \ldots \ldots \ldots \ldots \ldots \ldots \ldots$

IV CRITICAL VALUES OF $\alpha$ FOR $\theta=60^{\circ} \ldots \ldots \ldots \ldots$

V DOMINANT INSTABILITY FOR A CHOSEN VALUE OF $\alpha$ FOR $\theta=60^{\circ} \ldots \ldots \ldots \ldots \ldots \ldots \ldots \ldots \ldots \ldots \ldots \ldots$

VI $\quad$ CRITICAL VALUES OF $\alpha$ FOR $\theta=75^{\circ} \ldots \ldots \ldots$

VII DOMINANT INSTABILITY FOR A CHOSEN VALUE OF $\alpha$ FOR $\theta=75^{\circ} \ldots \ldots \ldots \ldots \ldots \ldots \ldots \ldots \ldots \ldots \ldots \ldots \ldots$ 


\section{LIST OF FIGURES}

FIGURE

PAGE

$1 \quad$ Surface plot of a Generalized Short-Crested Wave for $\frac{\rho_{1}}{\rho_{2}}=1 . \ldots \ldots$

2 Contour plot of a Generalized Short-Crested Wave for $\frac{\rho_{1}}{\rho_{2}}=1 \ldots \ldots . . \quad 57$

$3 \quad$ Surface plot of a Generalized Short-Crested Wave for $\frac{\rho_{1}}{\rho_{2}}=\frac{1}{2} \ldots \ldots . . \quad 58$

4 Contour plot of a Generalized Short-Crested Wave for $\frac{\rho_{1}}{\rho_{2}}=\frac{1}{2}$. . . . . $\quad 59$

$5 \quad$ Surface plot of a Generalized Short-Crested Wave for $\frac{\rho_{1}}{\rho_{2}}=\frac{1}{4} \ldots \ldots \ldots$

$6 \quad$ Contour plot of a Generalized Short-Crested Wave for $\frac{\rho_{1}}{\rho_{2}}=\frac{1}{4} . \ldots \ldots 1$

$7 \quad$ Stokes wave surface, $.04 \cos \left(k_{2} \cdot x\right) \ldots \ldots \ldots \ldots \ldots \ldots \ldots$

$8 \quad$ Stokes wave contours, $.04 \cos \left(k_{2} \cdot x\right) \ldots \ldots \ldots \ldots \ldots$

$9 \quad r_{\max }\left(45^{\circ}, \rho_{1}, \rho_{2} ; \alpha\right)$ versus wave height/slope $\alpha$ for $\frac{\rho_{1}}{\rho_{2}}=1 . \ldots \ldots$

$10 \quad r_{\max }\left(45^{\circ}, \rho_{1}, \rho_{2} ; \alpha\right)$ versus wave height/slope $\alpha$ for $\frac{\rho_{1}}{\rho_{2}}=\frac{1}{2} \ldots \ldots \ldots$

$11 r_{\max }\left(45^{\circ}, \rho_{1}, \rho_{2} ; \alpha\right)$ versus wave height/slope $\alpha$ for $\frac{\rho_{1}}{\rho_{2}}=\frac{1}{4} . \ldots \ldots$

$12 r_{\max }\left(45^{\circ}, \rho_{1}, \rho_{2} ; 0.015\right)$ versus quasi period $\left(p_{1}, p_{2}\right)$ for $\frac{\rho_{1}}{\rho_{2}}=1 \ldots \ldots$

$13 r_{\max }\left(45^{\circ}, \rho_{1}, \rho_{2} ; 0.015\right)$ versus quasi period $\left(p_{1}, p_{2}\right)$ for $\frac{\rho_{1}}{\rho_{2}}=\frac{1}{2} \ldots \ldots \quad 71$

$14 r_{\max }\left(45^{\circ}, \rho_{1}, \rho_{2} ; 0.010\right)$ versus quasi period $\left(p_{1}, p_{2}\right)$ for $\frac{\rho_{1}}{\rho_{2}}=\frac{1}{4} \ldots \ldots \quad 72$

$15 r_{\max }\left(60^{\circ}, \rho_{1}, \rho_{2} ; \alpha\right)$ versus wave height/slope $\alpha$ for $\frac{\rho_{1}}{\rho_{2}}=1 . \ldots \ldots . . . \quad 74$

$16 r_{\max }\left(60^{\circ}, \rho_{1}, \rho_{2} ; \alpha\right)$ versus wave height/slope $\alpha$ for $\frac{\rho_{1}}{\rho_{2}}=\frac{1}{2} \ldots \ldots \ldots$

$17 r_{\max }\left(60^{\circ}, \rho_{1}, \rho_{2} ; \alpha\right)$ versus wave height/slope $\alpha$ for $\frac{\rho_{1}}{\rho_{2}}=\frac{1}{4} \ldots \ldots \ldots$

$18 r_{\max }\left(60^{\circ}, \rho_{1}, \rho_{2} ; 0.020\right)$ versus quasi period $\left(p_{1}, p_{2}\right)$ for $\frac{\rho_{1}}{\rho_{2}}=1 \ldots \ldots$ 


\section{LIST OF FIGURES (Continued)}

\section{FIGURE}

PAGE

$19 r_{\max }\left(60^{\circ}, \rho_{1}, \rho_{2} ; 0.020\right)$ versus quasi period $\left(p_{1}, p_{2}\right)$ for $\frac{\rho_{1}}{\rho_{2}}=\frac{1}{2} \ldots \ldots$

$20 \quad r_{\max }\left(60^{\circ}, \rho_{1}, \rho_{2} ; 0.020\right)$ versus quasi period $\left(p_{1}, p_{2}\right)$ for $\frac{\rho_{1}}{\rho_{2}}=\frac{1}{4} \ldots \ldots$

$21 r_{\max }\left(75^{\circ}, \rho_{1}, \rho_{2} ; \alpha\right)$ versus wave height/slope $\alpha$ for $\frac{\rho_{1}}{\rho_{2}}=1 . \ldots \ldots$

$22 r_{\max }\left(75^{\circ}, \rho_{1}, \rho_{2} ; \alpha\right)$ versus wave height/slope $\alpha$ for $\frac{\rho_{1}}{\rho_{2}}=\frac{1}{2} . \ldots \ldots$

$23 \quad r_{\max }\left(75^{\circ}, \rho_{1}, \rho_{2} ; \alpha\right)$ versus wave height/slope $\alpha$ for $\frac{\rho_{1}}{\rho_{2}}=\frac{1}{4} . \ldots \ldots$

$24 r_{\max }\left(75^{\circ}, \rho_{1}, \rho_{2} ; 0.015\right)$ versus quasi period $\left(p_{1}, p_{2}\right)$ for $\frac{\rho_{1}}{\rho_{2}}=1 \ldots \ldots$

$25 \quad r_{\max }\left(75^{\circ}, \rho_{1}, \rho_{2} ; 0.015\right)$ versus quasi period $\left(p_{1}, p_{2}\right)$ for $\frac{\rho_{1}}{\rho_{2}}=\frac{1}{2} \ldots \ldots$

$26 \quad r_{\max }\left(75^{\circ}, \rho_{1}, \rho_{2} ; 0.015\right)$ versus quasi period $\left(p_{1}, p_{2}\right)$ for $\frac{\rho_{1}}{\rho_{2}}=\frac{1}{4} \ldots \ldots$ 


\section{LIST OF ABBREVIATIONS}

$\begin{array}{ll}\text { BIM/BEM } & \text { Boundary Integral/Element Methods } \\ \text { DNO } & \text { Dirichlet-Neumann Operator } \\ \text { FE } & \text { Field Expansions } \\ \text { GSCW } & \text { Generalized Short-Crested Wave } \\ \text { HOS } & \text { High-Order Spectral } \\ \text { LHS } & \text { Left Hand Side of a Given Equation } \\ \text { MSCS } & \text { Mathematics, Statistics, and Computer Science } \\ \text { RHS } & \text { Right Hand Side of a Given Equation } \\ \text { SCW } & \text { Short-Crested Wave } \\ \text { SIAM } & \text { Society For Industrial and Applied Mathematics } \\ \text { TFE } & \text { Transformed Field Expansions } \\ & \end{array}$




\section{SUMMARY}

We take up the question of the dynamic stability of genuinely two-dimensional generalized hexagonal traveling wave patterns on the surface of a three-dimensional ideal fluid. That is, the stability of Generalized Short-Crested Wave (GSCW) solutions of the water wave problem. Our study is restricted to spectral stability, which considers the linearization of the water wave operator about one of these traveling generalized hexagonal patterns. We draw conclusions about stability from the spectral data of the resulting linear operator.

Within the class of perturbations that we study, for a range of geometrical parameters, we find stable traveling waveforms which eventually destabilize, with features that depend strongly on the problem's configuration. We find Zones of Instability for patterns shaped as symmetric diamonds. Such zones are absent for asymmetric configurations; in these cases, once instability sets in, it remains. Within a given geometrical configuration, as a GSCW leading-order coefficient ratio is varied, these waves become more unstable as they become more asymmetric. 


\section{CHAPTER 1}

\section{INTRODUCTION}

The study of the movement of a large body of water under the influence of gravity, known as the water wave problem, is often accomplished by using the Euler equations of ideal fluid mechanics. These equations are a cornerstone of fluid mechanics modeling (1). The water wave problem lends itself to extensive use in a variety of applications, including tsunami propagation $(2 ; 3)$ and the transport of pollutants in the world's oceans $(4 ; 5)$. The use of linear approximations to study various problems has a rich history reaching back over 150 years (6).

Our study will focus on deep water and be restricted to the consideration of periodic traveling wave solutions. Such solutions are of interest because they transport energy and momentum over immense distances in the ocean (7). It is important to distinguish dynamically stable traveling waveforms from those that are not.

In particular, we will focus on the development of a surface evolution formulation of Euler's equations via the Dirichlet-Neumann operator. Moreover, we will examine numerical results arising from a linearization of this operator applied to Generalized Short-Crested Waves, which we take to be traveling surface shapes $\eta(x)$ with leading order behavior

$$
\eta_{1}(x)=\rho_{1} \cos \left(k_{1} \cdot x\right)+\rho_{2} \cos \left(k_{2} \cdot x\right)
$$


for linearly independent wave numbers $k_{1}, k_{2} \in \mathbf{R}^{2}$, where $\rho_{1}, \rho_{2} \in \mathbf{R}$ are both non-zero and positive.

If we let either $\rho_{1}=0$ or $\rho_{2}=0$ in (Equation 1.0.1), we have a Stokes wave. If $\rho_{1}=\rho_{2} \neq 0$, then we have a Short-Crested Wave. In this case, $\eta_{1}$ represents a diamond and $\eta$ becomes hexagonal as it becomes more nonlinear.

\subsection{Established Results for Short-Crested Waves}

The high-order numerical simulations of Roberts (8) and Roberts and Peregrine (9) began the investigations into SCWs and inspired a significant amount of further work. Nicholls and Craig contributed a rigorous theoretical analysis of their existence (10) with supporting numerical results (11). Further numerical work was done by Nicholls (12) for spectral continuation methods using parallel computing. Finally, Nicholls and Reitich developed a stable, high-order numerical algorithm for their computation (13) and a rigorous existence theory (14) as a result.

The results in the literature on the stability of SCWs are focused on spectral stability in the sense of the linearization of the water wave operator about one of these traveling hexagonal waves. Conclusions about stability are derived from the spectral data of this resulting linear operator. The definitive numerical simulations and results are found in the work of Ioualalen and Kharif for both superharmonic (15) and subharmonic (16) perturbations. The finite-depth work of Ioualalen, Karif, and their various collaborators $(17 ; 18 ; 19 ; 20)$ is also important.

The investigators for SCWs focused on resonant configurations of the wave numbers $k_{1}$ and $k_{2}$ in the traveling wave, with the wave numbers appearing in the leading order perturbation. For sufficiently small amplitudes, these linear resonances control the instability of the nonlinear 
traveling wave forms. Moreover, the nonlinear effects determine which perturbations are the strongest. When the amplitude is increased, other mechanisms for instability take over and move the strongest instabilities to other points, which can be far away from the linear resonance curves.

\subsection{Our Study of Generalized Short-Crested Waves}

Our conclusions about stability for GSCWs are driven by the spectral data of the resulting linear operator, but we focus on the role of asymmetry in terms of their geometric configuration via their underlying period, as well as their linear character through the leading-order coefficient

ratio $\frac{\rho_{1}}{\rho_{2}}$. We will also examine the interplay between the two. We draw conclusions on the question of stability, but we are not able to exhaust every possible unstable perturbation because of computational limitations. Thus the conclusions presented regarding instability are exact, while those for stability are sound but not complete.

In terms of geometric asymmetry, as the wave forms are skewed away from the symmetric diamond, waves that are similar in height become more unstable. Unexpectedly, in the symmetric case we found bands of wave heights that are unstable (Zones of Instability), yet there are stable wave forms of smaller and larger heights. This does not occur in the asymmetric geometries. For these, instability settles in at a particular wave height and remains for all greater wave heights.

In terms of the linear character, we found as the ratio $\frac{\rho_{1}}{\rho_{2}}$ decreases, the initial onset of instability occurs at smaller wave height values. 


\section{CHAPTER 2}

\section{EQUATIONS OF MOTION}

We study the motion of the free surface of an ideal (inviscid, irrotational, incompressible) three-dimensional (one vertical and two horizontal directions) deep fluid under the influence of gravity. The fluid occupies the domain

$$
S_{\eta}:=\left\{(x, y)=\left(x_{1}, x_{2}, y\right) \in \mathbf{R}^{2} \times \mathbf{R} \mid y<\eta(x, t)\right\}
$$

with free surface $\eta=\eta(x, t)$, velocity potential $\varphi=\varphi(x, y, t)(\mathbf{u}=\nabla \varphi)$, and gravitational constant $g$. The well-known equations of motion are (7)

$$
\begin{array}{ll}
\Delta \varphi=0 & \text { in } S_{\eta} \\
\partial_{y} \varphi \rightarrow 0 & y \rightarrow-\infty \\
\partial_{t} \eta=\partial_{y} \varphi-\nabla_{x} \eta \cdot \nabla_{x} \varphi & \text { at } y=\eta \\
\partial_{t} \varphi=-g \eta-\frac{1}{2} \nabla \varphi \cdot \nabla \varphi & \text { at } y=\eta,
\end{array}
$$

The initial conditions are

$$
\eta(x, 0)=\eta^{(0)}(x), \quad \varphi(x, \eta(x, 0), 0)=\xi^{(0)}(x),
$$


where by elliptic theory (21) we need to specify $\varphi$ only at the surface.

\section{$2.1 \quad$ Periodicity}

Boundary conditions are required for the study of the hexagonal waves that we undertake. Here they are periodicity with respect to some lattice

$$
\Gamma=\left\{\gamma \in \mathbf{R}^{2} \mid \gamma=m_{1} a_{1}+m_{2} a_{2} ; a_{1}, a_{2} \in \mathbf{R}^{2} ; m_{1}, m_{2} \in \mathbf{Z}\right\}
$$

generated by two linearly independent vectors $a_{1}, a_{2}$. Functions periodic with respect to $\Gamma$ satisfy

$$
\eta(x+\gamma, t)=\eta(x, t), \quad \varphi(x+\gamma, y, t)=\varphi(x, y, t), \quad \forall \gamma \in \Gamma
$$

and this lattice generates the conjugate lattice of wavenumbers

$$
\Gamma^{\prime}:=\left\{k \in \mathbf{R}^{2} \mid k \cdot \gamma \in(2 \pi) \mathbf{Z}, \forall \gamma \in \Gamma\right\}
$$

so that, for example, we can express $\eta$ by its Fourier series

$$
\eta(x, t)=\sum_{k \in \Gamma^{\prime}} \hat{\eta}_{k}(t) e^{i k \cdot x}
$$

Using the notation of Roberts et al $(22 ; 8 ; 9 ; 23)$ we focus our attention on Hexagonal or Short-Crested Waves (SCWs). Such waves are periodic both in the direction of propagation and the orthogonal horizontal direction. The period is set to $L_{1}:=L_{0} / \sin (\theta)$ in the direction parallel to propagation, and to $L_{2}:=L_{0} / \cos (\theta)$ in the direction orthogonal to propagation. 
Roberts used this to describe an $L_{0}$-periodic Stokes wave train incident upon a vertical wall where $\theta$ is the angle between the direction of propagation and the normal to the wall. Thus $\theta=0$ corresponds to Stokes waves while $\theta=\pi / 2$ represents standing waves. One can also think of SCWs as the interaction of two $L_{0}$-periodic Stokes wave trains incident upon one another at angle $\mu=\pi-2 \theta$. We choose the $x_{1}$-axis as the direction of propagation, thus the lattice of periodicity is

$$
\begin{aligned}
& \Gamma=\Gamma_{\theta}=\left\{\gamma \in \mathbf{R}^{2} \mid \gamma=m_{1} a_{1}+m_{2} a_{2} ;\right. \\
& \left.a_{1}=\left(\begin{array}{c}
L_{0} / \sin (\theta) \\
0
\end{array}\right), a_{2}=\left(\begin{array}{c}
0 \\
L_{0} / \cos (\theta)
\end{array}\right) ; m_{1}, m_{2} \in \mathbf{Z}\right\},
\end{aligned}
$$

and the conjugate lattice is given by

$$
\begin{aligned}
& \Gamma^{\prime}=\Gamma_{\theta}^{\prime}=\left\{k \in \mathbf{R}^{2} \mid\right. k=m_{1} b_{1}+m_{2} b_{2} ; \\
&\left.b_{1}=\left(\begin{array}{c}
2 \pi \sin (\theta) / L_{0} \\
0
\end{array}\right), b_{2}=\left(\begin{array}{c}
0 \\
2 \pi \cos (\theta) / L_{0}
\end{array}\right) ; m_{1}, m_{2} \in \mathbf{Z}\right\} .
\end{aligned}
$$

\subsection{Surface Formulation}

A simplification of the formulation of (Equation 2.0.1) and a reduction in dimension (by restricting to the surface of the water) was realized by Craig and Sulem (24) with the DirichletNeumann operator. This operator maps Dirichlet data to Neumann data. In the work of Nicholls (12) we find this applied to spectral stability. 
Definition 2.2.1. Let $N_{\eta}=\left(-\nabla_{x} \eta, 1\right)$, where $\eta(x, t)$ is the free surface and $\varphi(x, y, t)$ is the velocity potential. Then the Dirichlet-Neumann operator (DNO) is defined as

$$
G(\eta)[\xi]:=\left.\nabla \varphi\right|_{y=\eta} \cdot N_{\eta}=-\left.\nabla_{x} \eta \cdot \nabla_{x} \varphi\right|_{y=\eta}+\left.\partial_{y} \varphi\right|_{y=\eta}
$$

where $\xi(x, t):=\varphi(x, \eta(x, t), t)$.

Proposition 2.2.1. The water wave problem (Equation 2.0.1) can be equivalently stated as the following surface evolution problem

$$
\begin{aligned}
& \partial_{t} \eta=G(\eta)[\xi] \\
& \partial_{t} \xi=-g \eta-A(\eta) B(\eta, \xi),
\end{aligned}
$$

where

$$
\begin{aligned}
A(\eta):= & \frac{1}{2\left(1+\nabla_{x} \eta \cdot \nabla_{x} \eta\right)} \\
B(\eta, \xi):= & \nabla_{x} \xi \cdot \nabla_{x} \xi-(G(\eta)[\xi])^{2}-2(G(\eta)[\xi]) \nabla_{x} \xi \cdot \nabla_{x} \eta \\
& +\left(\nabla_{x} \xi \cdot \nabla_{x} \xi\right)\left(\nabla_{x} \eta \cdot \nabla_{x} \eta\right)-\left(\nabla_{x} \xi \cdot \nabla_{x} \eta\right)^{2} .
\end{aligned}
$$

Proof. (Equation 2.2.2a) is immediate from the Kinematic condition (Equation 2.0.1c). To demonstrate (Equation 2.2.2b), we eliminate the Bernoulli condition's (Equation 2.0.1d) dependence on $\varphi$. 
By the chain rule we have

$$
\partial_{t} \xi=\left(\left.\partial_{y} \varphi\right|_{y=\eta}\right)\left(\partial_{t} \eta\right)+\left.\partial_{t} \varphi\right|_{y=\eta}
$$

which yields

$$
\left.\partial_{t} \varphi\right|_{y=\eta}=\partial_{t} \xi-\left(\left.\partial_{y} \varphi\right|_{y=\eta}\right)\left(\partial_{t} \eta\right)
$$

Applying the chain rule component-wise yields

$$
\left.\nabla_{x} \varphi\right|_{y=\eta}=\nabla_{x} \xi-\left(\left.\partial_{y} \varphi\right|_{y=\eta}\right) \nabla_{x} \eta
$$

A rearrangement of the definition of the DNO (Equation 2.2.1) tells us

$$
\left.\partial_{y} \varphi\right|_{y=\eta}=G(\eta)[\xi]+\left.\nabla_{x} \eta \cdot \nabla_{x} \varphi\right|_{y=\eta}
$$

Substituting the result for $\left.\nabla_{x} \varphi\right|_{y=\eta}$ from (Equation 2.2.5) into (Equation 2.2.6) gives us

$$
\left.\partial_{y} \varphi\right|_{y=\eta}=G(\eta)[\xi]+\nabla_{x} \xi \cdot \nabla_{x} \eta-\left(\left.\partial_{y} \varphi\right|_{y=\eta}\right)\left(\nabla_{x} \eta \cdot \nabla_{x} \eta\right)
$$

We now collect like terms and solve for $\left.\partial_{y} \varphi\right|_{y=\eta}$ to find

$$
\left.\partial_{y} \varphi\right|_{y=\eta}=\frac{1}{\left|N_{\eta}\right|^{2}}\left(G(\eta)[\xi]+\nabla_{x} \xi \cdot \nabla_{x} \eta\right)
$$


Returning to (Equation 2.0.1d) and substituting in the RHS of each of (Equation 2.2.4) and (Equation 2.2.5) gives us

$$
\begin{aligned}
\partial_{t} \xi-\left(\left.\partial_{y} \varphi\right|_{y=\eta}\right)\left(\partial_{t} \eta\right)= & -g \eta-\frac{1}{2} \nabla \varphi \cdot \nabla \varphi \\
= & -g \eta-\frac{1}{2} \nabla_{x} \varphi \cdot \nabla_{x} \varphi-\frac{1}{2}\left(\left.\partial_{y} \varphi\right|_{y=\eta}\right)^{2} \\
= & -g \eta-\frac{1}{2}\left(\nabla_{x} \xi-\left(\left.\partial_{y} \varphi\right|_{y=\eta}\right) \nabla_{x} \eta\right) \cdot\left(\nabla_{x} \xi-\left(\left.\partial_{y} \varphi\right|_{y=\eta}\right) \nabla_{x} \eta\right)-\frac{1}{2}\left(\left.\partial_{y} \varphi\right|_{y=\eta}\right)^{2} \\
= & -g \eta-\frac{1}{2}\left(\nabla_{x} \xi \cdot \nabla_{x} \xi-2\left(\left.\partial_{y} \varphi\right|_{y=\eta}\right)\left(\nabla_{x} \eta \cdot \nabla_{x} \xi\right)+\left(\left.\partial_{y} \varphi\right|_{y=\eta}\right)^{2}\left(\nabla_{x} \eta \cdot \nabla_{x} \eta\right)\right) \\
& -\frac{1}{2}\left(\left.\partial_{y} \varphi\right|_{y=\eta}\right)^{2} \\
= & -g \eta-\frac{1}{2} \nabla_{x} \xi \cdot \nabla_{x} \xi+\left(\left.\partial_{y} \varphi\right|_{y=\eta}\right)\left(\nabla_{x} \eta \cdot \nabla_{x} \xi\right)-\frac{1}{2}\left(\left.\partial_{y} \varphi\right|_{y=\eta}\right)^{2}\left(\nabla_{x} \eta \cdot \nabla_{x} \eta\right) \\
& -\frac{1}{2}\left(\left.\partial_{y} \varphi\right|_{y=\eta}\right)^{2}
\end{aligned}
$$

Moving $\left(\left.\partial_{y} \varphi\right|_{y=\eta}\right)\left(\partial_{t} \eta\right)$ to the RHS of the (Equation 2.2.8) and using (Equation 2.2.2a) gives us

$$
\begin{aligned}
\partial_{t} \xi & =\left(\left.\partial_{y} \varphi\right|_{y=\eta}\right)(G(\eta)[\xi])-g \eta-\frac{1}{2} \nabla_{x} \xi \cdot \nabla_{x} \xi+\left(\left.\partial_{y} \varphi\right|_{y=\eta}\right)\left(\nabla_{x} \eta \cdot \nabla_{x} \xi\right)-\frac{1}{2}\left|N_{\eta}\right|^{2}\left(\left.\partial_{y} \varphi\right|_{y=\eta}\right)^{2} \\
& =-g \eta-\frac{1}{2} \nabla_{x} \xi \cdot \nabla_{x} \xi+\left(G(\eta)[\xi]+\nabla_{x} \eta \cdot \nabla_{x} \xi\right)\left(\left.\partial_{y} \varphi\right|_{y=\eta}\right)-\frac{1}{2}\left|N_{\eta}\right|^{2}\left(\left.\partial_{y} \varphi\right|_{y=\eta}\right)^{2} .
\end{aligned}
$$


Finally, substituting (Equation 2.2.7) into (Equation 2.2.9) and simplifying a bit tells us

$$
\begin{aligned}
\partial_{t} \xi= & -g \eta-\frac{1}{2} \nabla_{x} \xi \cdot \nabla_{x} \xi+\frac{1}{2\left|N_{\eta}\right|^{2}}\left(G(\eta)[\xi]+\nabla_{x} \eta \cdot \nabla_{x} \xi\right)^{2} \\
= & -g \eta+\frac{-\left(\nabla_{x} \xi \cdot \nabla_{x} \xi\right)\left|N_{\eta}\right|^{2}+\left(G(\eta)[\xi]+\nabla_{x} \eta \cdot \nabla_{x} \xi\right)^{2}}{2\left|N_{\eta}\right|^{2}} \\
= & -g \eta+\frac{-\nabla_{x} \xi \cdot \nabla_{x} \xi-\left(\nabla_{x} \xi \cdot \nabla_{x} \xi\right)\left(\nabla_{x} \eta \cdot \nabla_{x} \eta\right)+(G(\eta)[\xi])^{2}}{2\left|N_{\eta}\right|^{2}} \\
& +\frac{2(G(\eta)[\xi])\left(\nabla_{x} \eta \cdot \nabla_{x} \xi\right)+\left(\nabla_{x} \eta \cdot \nabla_{x} \xi\right)^{2}}{2\left|N_{\eta}\right|^{2}} \\
= & -g \eta-\frac{\left.\left(\nabla_{x} \xi \cdot \nabla_{x} \xi+\left(\nabla_{x} \xi \cdot \nabla_{x} \xi\right)\left(\nabla_{x} \eta \cdot \nabla_{x} \eta\right)-(G(\eta)[\xi])^{2}\right)\right)}{2\left|N_{\eta}\right|^{2}} \\
& \quad-\frac{\left(-2(G(\eta)[\xi])\left(\nabla_{x} \eta \cdot \nabla_{x} \xi\right)-\left(\nabla_{x} \eta \cdot \nabla_{x} \xi\right)^{2}\right)}{2\left|N_{\eta}\right|^{2}}
\end{aligned}
$$

which yields (Equation 2.2.2b) and the expressions for $A(\eta)$ and $B(\eta, \xi)$.

\subsection{The First Variations of $A(\eta)$ and $B(\eta, \xi)$}

For our spectral stability analysis, we need the first variations of $A(\eta)$ and $B(\eta, \xi)$. 
Proposition 2.3.1. Given $\eta$ and $\xi$ as defined in Definition 2.2.1, the first variations of $A(\eta)$ and $B(\eta, \xi)$ are:

$$
\begin{aligned}
A_{\eta}(\eta)\{v\}= & -\frac{\nabla_{x} \eta \cdot \nabla_{x} v}{\left(1+\nabla_{x} \eta \cdot \nabla_{x} \eta\right)^{2}} \\
B_{\eta}(\eta, \xi)\{v\}= & -2(G(\eta)[\xi])\left(G_{\eta}(\eta)[\xi]\{v\}\right)-2\left(G_{\eta}(\eta)[\xi]\{v\}\right)\left(\nabla_{x} \xi \cdot \nabla_{x} \eta\right) \\
& -2(G(\eta)[\xi])\left(\nabla_{x} \xi \cdot \nabla_{x} v\right)+2\left(\nabla_{x} \xi \cdot \nabla_{x} \xi\right)\left(\nabla_{x} \eta \cdot \nabla_{x} v\right) \\
& -2\left(\nabla_{x} \xi \cdot \nabla_{x} \eta\right)\left(\nabla_{x} \xi \cdot \nabla_{x} v\right) \\
B_{\xi}(\eta, \xi)\{v\}= & 2 \nabla_{x} \xi \cdot \nabla_{x} v-2(G(\eta)[\xi])(G(\eta)[v])-2(G(\eta)[v])\left(\nabla_{x} \xi \cdot \nabla_{x} \eta\right) \\
& -2(G(\eta)[\xi])\left(\nabla_{x} v \cdot \nabla_{x} \eta\right)+2\left(\nabla_{x} \xi \cdot \nabla_{x} v\right)\left(\nabla_{x} \eta \cdot \nabla_{x} \eta\right) \\
& -2\left(\nabla_{x} \xi \cdot \nabla_{x} \eta\right)\left(\nabla_{x} v \cdot \nabla_{x} \eta\right)
\end{aligned}
$$

where $G_{\eta}(\eta)$ is the first variation of $G(\eta)$.

Proof. For (Equation 2.3.1): By definition

$$
A_{\eta}(\eta)\{v\}=\lim _{\epsilon \rightarrow 0} \frac{A(\eta+\epsilon v)-A(\eta)}{\epsilon}
$$




$$
\begin{aligned}
\frac{A(\eta+\epsilon v)-A(\eta)}{\epsilon} & =\frac{1}{2 \epsilon}\left(\frac{1}{\left(1+\nabla_{x}(\eta+\epsilon v) \cdot \nabla_{x}(\eta+\epsilon v)\right)}-\frac{1}{\left(1+\nabla_{x} \eta \cdot \nabla_{x} \eta\right)}\right) \\
& =\frac{1}{2 \epsilon}\left(\frac{\nabla_{x} \eta \cdot \nabla_{x} \eta-\nabla_{x}(\eta+\epsilon v) \cdot \nabla_{x}(\eta+\epsilon v)}{\left(1+\nabla_{x}(\eta+\epsilon v) \cdot \nabla_{x}(\eta+\epsilon v)\right) \cdot\left(1+\nabla_{x} \eta \cdot \nabla_{x} \eta\right)}\right) \\
& =\frac{1}{2 \epsilon}\left(\frac{-2 \epsilon \nabla_{x} \eta \cdot \nabla_{x} v-\epsilon^{2} \nabla_{x} v \cdot \nabla_{x} v}{\left(1+\nabla_{x}(\eta+\epsilon v) \cdot \nabla_{x}(\eta+\epsilon v)\right) \cdot\left(1+\nabla_{x} \eta \cdot \nabla_{x} \eta\right)}\right) \\
& =\frac{-\nabla_{x} \eta \cdot \nabla_{x} v-\frac{1}{2} \epsilon \nabla_{x} v \cdot \nabla_{x} v}{\left(1+\nabla_{x}(\eta+\epsilon v) \cdot \nabla_{x}(\eta+\epsilon v)\right) \cdot\left(1+\nabla_{x} \eta \cdot \nabla_{x} \eta\right)}
\end{aligned}
$$

Thus

$$
A_{\eta}(\eta)\{v\}=-\frac{\nabla_{x} \eta \cdot \nabla_{x} v}{\left(1+\nabla_{x} \eta \cdot \nabla_{x} \eta\right)^{2}} .
$$

For (Equation 2.3.2): Using the definition, canceling out $\nabla_{x} \xi \cdot \nabla_{x} \xi$, and doing a little judicious grouping gives us

$$
\begin{aligned}
B_{\eta}(\eta, \xi)\{v\}= & \lim _{\epsilon \rightarrow 0} \frac{B(\eta+\epsilon v, \xi)-B(\eta, \xi)}{\epsilon} \\
=- & \lim _{\epsilon \rightarrow 0} \frac{(G(\eta+\epsilon v)[\xi])^{2}-(G(\eta)[\xi])^{2}}{\epsilon} \\
& -2 \cdot \lim _{\epsilon \rightarrow 0} \frac{G(\eta+\epsilon v)[\xi]\left(\nabla_{x} \xi \cdot \nabla_{x}(\eta+\epsilon v)\right)-G(\eta)[\xi]\left(\nabla_{x} \xi \cdot \nabla_{x} \eta\right)}{\epsilon} \\
& +\lim _{\epsilon \rightarrow 0} \frac{\left(\nabla_{x} \xi \cdot \nabla_{x} \xi\right)\left(\nabla_{x}(\eta+\epsilon v) \cdot \nabla_{x}(\eta+\epsilon v)\right)-\left(\nabla_{x} \xi \cdot \nabla_{x} \xi\right)\left(\nabla_{x} \eta \cdot \nabla_{x} \eta\right)}{\epsilon} \\
& -\lim _{\epsilon \rightarrow 0} \frac{\left(\nabla_{x} \xi \cdot \nabla_{x}(\eta+\epsilon v)\right)^{2}-\left(\nabla_{x} \xi \cdot \nabla_{x} \eta\right)^{2}}{\epsilon} .
\end{aligned}
$$


We now break down each of the above limits.

$$
\begin{aligned}
(\text { Equation 2.3.4) } & =-\lim _{\epsilon \rightarrow 0}(G(\eta+\epsilon v)[\xi]+G(\eta)[\xi]) \cdot\left(\frac{G(\eta+\epsilon v)[\xi]-G(\eta)[\xi]}{\epsilon}\right) \\
& =-2(G(\eta)[\xi])\left(G_{\eta}(\eta)[\xi]\{v\}\right) .
\end{aligned}
$$

$$
\begin{aligned}
\text { (Equation 2.3.5) } & =-2 \cdot \lim _{\epsilon \rightarrow 0} \frac{G(\eta+\epsilon v)[\xi]\left(\nabla_{x} \xi \cdot \nabla_{x} \eta\right)+\epsilon G(\eta+\epsilon v)[\xi]\left(\nabla_{x} \xi \cdot \nabla_{x} v\right)-G(\eta)[\xi]\left(\nabla_{x} \xi \cdot \nabla_{x} \eta\right)}{\epsilon} \\
& =-2\left(\nabla_{x} \xi \cdot \nabla_{x} \eta\right) \cdot \lim _{\epsilon \rightarrow 0} \frac{G(\eta+\epsilon v)[\xi]-G(\eta)[\xi]}{\epsilon}-2\left(\nabla_{x} \xi \cdot \nabla_{x} v\right) \cdot \lim _{\epsilon \rightarrow 0} G(\eta+\epsilon v)[\xi] \\
& =-2 G_{\eta}(\eta)[\xi]\{v\}\left(\nabla_{x} \xi \cdot \nabla_{x} \eta\right)-2 G(\eta)[\xi]\left(\nabla_{x} \xi \cdot \nabla_{x} v\right) .
\end{aligned}
$$

$$
\begin{aligned}
\text { (Equation 2.3.6) }= & \lim _{\epsilon \rightarrow 0} \frac{\left(\nabla_{x} \xi \cdot \nabla_{x} \xi\right)\left(\nabla_{x} \eta \cdot\left(\nabla_{x} \eta+\epsilon \nabla_{x} v\right)+\epsilon \nabla_{x} v \cdot\left(\nabla_{x} \eta+\epsilon \nabla_{x} v\right)\right)}{\epsilon} \\
& -\frac{\left(\nabla_{x} \xi \cdot \nabla_{x} \xi\right)\left(\nabla_{x} \eta \cdot \nabla_{x} \eta\right)}{\epsilon} \\
= & \lim _{\epsilon \rightarrow 0} \frac{\left(\nabla_{x} \xi \cdot \nabla_{x} \xi\right)\left(\nabla_{x} \eta \cdot \nabla_{x} \eta+2 \epsilon \nabla_{x} \eta \cdot \nabla_{x} v+\epsilon^{2} \nabla_{x} v \cdot \nabla_{x} v\right)}{\epsilon} \\
& -\frac{\left(\nabla_{x} \xi \cdot \nabla_{x} \xi\right)\left(\nabla_{x} \eta \cdot \nabla_{x} \eta\right)}{\epsilon} \\
= & \lim _{\epsilon \rightarrow 0}\left(2\left(\nabla_{x} \eta \cdot \nabla_{x} \eta\right)\left(\nabla_{x} \eta \cdot \nabla_{x} v\right)+\epsilon \nabla_{x} v \cdot \nabla_{x} v\right) \\
= & 2\left(\nabla_{x} \eta \cdot \nabla_{x} \eta\right)\left(\nabla_{x} \eta \cdot \nabla_{x} v\right) .
\end{aligned}
$$




$$
\begin{aligned}
(\text { Equation 2.3.7) } & =-\lim _{\epsilon \rightarrow 0} \frac{\left(\nabla_{x} \xi \cdot \nabla_{x} \eta+\epsilon \nabla_{x} \xi \cdot \nabla_{x} v\right)^{2}-\left(\nabla_{x} \xi \cdot \nabla_{x} \eta\right)^{2}}{\epsilon} \\
& =-\lim _{\epsilon \rightarrow 0} \frac{\left(\nabla_{x} \xi \cdot \nabla_{x} \eta\right)^{2}+2 \epsilon\left(\nabla_{x} \xi \cdot \nabla_{x} \eta\right)\left(\nabla_{x} \xi \cdot \nabla_{x} v\right)-\left(\nabla_{x} \xi \cdot \nabla_{x} \eta\right)^{2}}{\epsilon} \\
& =-2\left(\nabla_{x} \xi \cdot \nabla_{x} \eta\right)\left(\nabla_{x} \xi \cdot \nabla_{x} v\right) .
\end{aligned}
$$

Putting together (Equation 2.3.8), (Equation 2.3.9), (Equation 2.3.10), and (Equation 2.3.11) gives us $B_{\eta}(\eta, \xi)[\xi]\{v\}$ as in (Equation 2.3.2).

For (Equation 2.3.3): Using the definition and doing a little judicious grouping gives us

$$
\begin{aligned}
B_{\xi}(\eta, \xi)\{v\}= & \lim _{\epsilon \rightarrow 0} \frac{B(\eta, \xi+\epsilon v)-B(\eta, \xi)}{\epsilon} \\
= & \lim _{\epsilon \rightarrow 0} \frac{\nabla_{x}(\xi+\epsilon v) \cdot \nabla_{x}(\xi+\epsilon v)-\nabla_{x} \xi \cdot \nabla_{x} \xi}{\epsilon} \\
& -\lim _{\epsilon \rightarrow 0} \frac{(G(\eta)[\xi+\epsilon v])^{2}-(G(\eta)[\xi])^{2}}{\epsilon} \\
& -2 \lim _{\epsilon \rightarrow 0} \frac{(G(\eta)[\xi+\epsilon v])\left(\nabla_{x}(\xi+\epsilon v) \cdot \nabla_{x} \eta\right)-G(\eta)[\xi]\left(\nabla_{x} \xi \cdot \nabla_{x} \eta\right)}{\epsilon} \\
& +\lim _{\epsilon \rightarrow 0} \frac{\left(\nabla_{x}(\xi+\epsilon v) \cdot \nabla_{x}(\xi+\epsilon v)\right)\left(\nabla_{x} \eta \cdot \nabla_{x} \eta\right)-\left(\nabla_{x} \xi \cdot \nabla_{x} \xi\right)\left(\nabla_{x} \eta \cdot \nabla_{x} \eta\right)}{\epsilon} \\
& -\lim _{\epsilon \rightarrow 0} \frac{\left(\nabla_{x}(\xi+\epsilon v) \cdot \nabla_{x} \eta\right)^{2}-\left(\nabla_{x} \xi \cdot \nabla_{x} \eta\right)^{2}}{\epsilon} .
\end{aligned}
$$


We now break down the above limits to determine $B_{\xi}(\eta, \xi)\{v\}$.

$$
\begin{aligned}
(\text { Equation 2.3.12) } & =\lim _{\epsilon \rightarrow 0} \frac{\nabla_{x} \xi \cdot \nabla_{x} \xi+2 \epsilon \nabla_{x} \xi \cdot \nabla_{x} v+\epsilon^{2} \nabla_{x} v \cdot \nabla_{x} v-\nabla_{x} \xi \cdot \nabla_{x} \xi}{\epsilon} \\
& =2 \nabla_{x} \xi \cdot \nabla_{x} v+\lim _{\epsilon \rightarrow 0} \epsilon \nabla_{x} v \cdot \nabla_{x} v \\
& =2 \nabla_{x} \xi \cdot \nabla_{x} v .
\end{aligned}
$$

$$
\begin{aligned}
\text { (Equation 2.3.13) } & =-\lim _{\epsilon \rightarrow 0} \frac{(G(\eta)[\xi+\epsilon v]-G(\eta)[\xi]) \cdot(G(\eta)[\xi+\epsilon v]+G(\eta)[\xi])}{\epsilon} \\
& =-\lim _{\epsilon \rightarrow 0} \frac{(G(\eta)[\xi]+\epsilon G(\eta)[v]-G(\eta)[\xi]) \cdot(G(\eta)[\xi]+\epsilon G(\eta)[v]+G(\eta)[\xi])}{\epsilon} \\
& =-2(G(\eta)[\xi])(G(\eta)[v]) .
\end{aligned}
$$

(Equation 2.3.14) $=-2 \lim _{\epsilon \rightarrow 0} \frac{G(\eta)[\xi+\epsilon v]\left(\nabla_{x} \xi \cdot \nabla_{x} \eta+\epsilon \nabla_{x} v \cdot \nabla_{x} \eta\right)-G(\eta)[\xi]\left(\nabla_{x} \xi \cdot \nabla_{x} \eta\right)}{\epsilon}$

$$
\begin{aligned}
& =-2 \lim _{\epsilon \rightarrow 0} \frac{(G(\eta)[\xi]+\epsilon G(\eta)[v])\left(\nabla_{x} \xi \cdot \nabla_{x} \eta+\epsilon \nabla_{x} v \cdot \nabla_{x} \eta\right)-G(\eta)[\xi]\left(\nabla_{x} \xi \cdot \nabla_{x} \eta\right)}{\epsilon} \\
& =-2(G(\eta)[v])\left(\nabla_{x} \xi \cdot \nabla_{x} \eta\right)-2(G(\eta)[\xi])\left(\nabla_{x} v \cdot \nabla_{x} \eta\right)-2 \lim _{\epsilon \rightarrow 0} \epsilon(G(\eta)[v])\left(\nabla_{x} v \cdot \nabla_{x} \eta\right) \\
& =-2(G(\eta)[v])\left(\nabla_{x} \xi \cdot \nabla_{x} \eta\right)-2(G(\eta)[\xi])\left(\nabla_{x} v \cdot \nabla_{x} \eta\right) .
\end{aligned}
$$

(Equation 2.3.15) $=\left(\nabla_{x} \eta \cdot \nabla_{x} \eta\right) \lim _{\epsilon \rightarrow 0} \frac{\left(\nabla_{x} \xi+\epsilon \nabla_{x} v\right) \cdot \nabla_{x} \xi+\epsilon\left(\nabla_{x} \xi+\epsilon \nabla_{x} v\right) \cdot \nabla_{x} v-\nabla_{x} \xi \cdot \nabla_{x} \xi}{\epsilon}$

$$
\begin{aligned}
& =\left(\nabla_{x} \eta \cdot \nabla_{x} \eta\right) \lim _{\epsilon \rightarrow 0} \frac{2 \epsilon \nabla_{x} \xi \cdot \nabla_{x} v+\epsilon^{2} \nabla_{x} v \cdot \nabla_{x} v}{\epsilon} \\
& =2\left(\nabla_{x} \xi \cdot \nabla_{x} v\right)\left(\nabla_{x} \eta \cdot \nabla_{x} \eta\right) .
\end{aligned}
$$




$$
\begin{aligned}
(\text { Equation 2.3.16) } & =-\lim _{\epsilon \rightarrow 0} \frac{\left(\nabla_{x} \xi \cdot \nabla_{x} \eta+\epsilon \nabla_{x} v \cdot \nabla_{x} \eta\right)^{2}-\left(\nabla_{x} \xi \cdot \nabla_{x} \eta\right)^{2}}{\epsilon} \\
& =-\lim _{\epsilon \rightarrow 0} \frac{2 \epsilon\left(\nabla_{x} \xi \cdot \nabla_{x} \eta\right)\left(\nabla_{x} v \cdot \nabla_{x} \eta\right)+\epsilon^{2}\left(\nabla_{x} v \cdot \nabla_{x} \eta\right)^{2}}{\epsilon} \\
& =-2\left(\nabla_{x} \xi \cdot \nabla_{x} \eta\right)\left(\nabla_{x} v \cdot \nabla_{x} \eta\right) .
\end{aligned}
$$

Putting together (Equation 2.3.17), (Equation 2.3.18), (Equation 2.3.19), (Equation 2.3.20), and (Equation 2.3.21) gives us $B_{\xi}(\eta, \xi)[\xi]\{v\}$ as in (Equation 2.3.3).

\subsection{The Hamiltonian}

Zakharov (25) showed that the system (Equation 2.0.1) is Hamiltonian in terms of the canonical variables $\eta(x, t)$ and $\xi(x, t)$ with Hamiltonian

$$
H_{Z}=\frac{1}{2} \int_{P(\Gamma)}\left(\int_{-\infty}^{\eta} \nabla \varphi \cdot \nabla \varphi \mathrm{d} y\right)+g \eta^{2} \mathrm{~d} x
$$

where $P(\Gamma)$ is the period cell associated to $\Gamma$. It is natural to rewrite this in the terms of our surface formulation.

Proposition 2.4.1. The Hamiltonian $H_{Z}$ can be equivalently written as

$$
H_{Z C S}=\frac{1}{2} \int_{P(\Gamma)} \xi(G(\eta)[\xi])+g \eta^{2} d x
$$


Proof. Using an identity from vector calculus to rewrite $\nabla \varphi \cdot \nabla \varphi$, the fact $\Delta \varphi=\operatorname{div}[\nabla \varphi]=0$ in $S_{\eta}$, and Gauss' theorem with $n_{h}=(0,0,-1)$ and $n_{\eta}=\frac{N_{\eta}}{\left|N_{\eta}\right|}$ we have

$$
\begin{aligned}
\int_{P(\Gamma)}\left(\int_{-\infty}^{\eta} \nabla \varphi \cdot \nabla \varphi \mathrm{d} y\right) \mathrm{d} x & =\int_{P(\Gamma)}\left(\int_{-\infty}^{\eta}(\operatorname{div}[\varphi \nabla \varphi]-\varphi \cdot \operatorname{div}[\nabla \varphi]) \mathrm{d} y\right) \mathrm{d} x \\
& =\int_{P(\Gamma)} \int_{-\infty}^{\eta} \operatorname{div}[\varphi \nabla \varphi] \mathrm{d} y \mathrm{~d} x \\
& =\lim _{h \rightarrow \infty} \int_{P(\Gamma)} \int_{-h}^{\eta} \operatorname{div}[\varphi \nabla \varphi] \mathrm{d} y \mathrm{~d} x \\
& =\lim _{h \rightarrow \infty} \int_{y=-h} \varphi\left(\nabla \varphi \cdot n_{h}\right) \mathrm{d} S+\int_{y=\eta} \varphi\left(\nabla \varphi \cdot n_{\eta}\right) \mathrm{d} S \\
& =\lim _{h \rightarrow \infty} \int_{P(\Gamma)}-\varphi \cdot \partial_{y} \varphi \mathrm{d} x+\int_{P(\Gamma)} \xi\left(\left.\nabla \varphi\right|_{y=\eta} \cdot N_{\eta}\right) \mathrm{d} x \\
& =\int_{P(\Gamma)} \xi(G(\eta)[\xi]) \mathrm{d} x
\end{aligned}
$$

using the definition of $\xi$ and $G(\eta)[\xi]$, (Equation 2.0.1b), and $\mathrm{d} x=\frac{\mathrm{d} S}{\left|N_{\eta}\right|}$. Therefore,

$$
H_{z}=\frac{1}{2} \int_{P(\Gamma)} \xi(G(\eta)[\xi])+g \eta^{2} \mathrm{~d} x
$$

\subsection{Traveling Wave Evolution Equations}

Our focus is upon the stability of traveling wave solutions of (Equation 2.2.2) and so we move to a reference frame moving uniformly with velocity $c=\left(c_{1}, c_{2}\right) \in \mathbf{R}^{2}$. Let $\tilde{x}=\left(\tilde{x_{1}}, \tilde{x_{2}}\right)=$ $\left(x_{1}-c_{1} t, x_{2}-c_{2} t\right), \quad \tilde{y}=y, \quad \tilde{t}=t, \quad \bar{\eta}(\tilde{x}, \tilde{t})=\eta(\tilde{x}-c t, t), \quad \bar{\varphi}(\tilde{x}, \tilde{y}, \tilde{t})=\varphi(\tilde{x}-c t, y, t)$, and $\bar{\xi}=\tilde{\varphi}(\tilde{x}, \tilde{\eta}(\tilde{x}, \tilde{t}), \tilde{t})$ 
By simple applications of the chain rule, we have

$$
\nabla_{\tilde{x}}=\nabla_{x}
$$

and

$$
\partial_{t}=\partial_{\tilde{t}}-c \cdot \nabla_{\tilde{x}}
$$

Applying the above by referring back to Proposition 2.2.1, and dropping the tildes and bars, we have the governing equations for a uniformly moving reference frame:

$$
\begin{aligned}
& \partial_{t} \eta+c \cdot \nabla_{x} \eta=G(\eta)[\xi] \\
& \partial_{t} \xi+c \cdot \nabla_{x} \xi=-g \eta-A(\eta) B(\eta, \xi) .
\end{aligned}
$$

\subsection{Dimensionless Variables}

We non-dimensionalize our variables to facilitate our computations and simulations. We use the classical scalings of Lamb (7).

Definition 2.6.1. Let

$$
x_{1}=L x_{1}^{\prime}, \quad x_{2}=L x_{2}^{\prime}, \quad y=L y^{\prime}, \quad \eta=a \eta^{\prime}, \quad \xi=X \xi^{\prime}, \quad t=T t^{\prime},
$$


where

$$
X=a \sqrt{L g}, \quad T=\sqrt{\frac{L}{g}}, \quad L=\frac{L_{0}}{2 \pi} .
$$

Recall from $\S 2.1$ that the traveling wave has wavelengths $L_{1}=\frac{L_{0}}{\sin (\theta)}$ and $L_{2}=\frac{L_{0}}{\cos (\theta)}$ in the $x_{1}$ and $x_{2}$ directions, respectively. It is clear from the scalings above and applications of the chain rule that

$$
\nabla_{x}=\frac{1}{L} \nabla_{x^{\prime}}, \quad \partial_{t}=\frac{1}{T} \partial_{t^{\prime}}
$$

We recommend the reader peruse Chapter 3 at this point before continuing with this chapter in order to gain an understanding of the series expansion for the DNO $G(\eta)[\xi]$.

Proposition 2.6.1. Let $\alpha:=\frac{a}{L}$ and $D^{\prime}:=\frac{1}{i} \partial_{x^{\prime}}$. Given Definition 2.6.1, the operator expansions for $G(\eta), A(\eta)$, and $B(\eta, \xi)$ become

$$
\begin{aligned}
G\left(a \eta^{\prime}\right) & =\frac{1}{L} \sum_{n=0}^{\infty} G_{n}^{\prime}\left(\eta^{\prime}\right) \alpha^{n} \\
A\left(a \eta^{\prime}\right) & =\sum_{n=0}^{\infty} A_{n}^{\prime}\left(\eta^{\prime}\right) \alpha^{n} \\
B\left(a \eta^{\prime}, X \xi^{\prime}\right) & =\frac{X}{T} \sum_{n=1}^{\infty} B_{n}^{\prime}\left(\eta^{\prime}, \xi^{\prime}\right) \alpha^{n},
\end{aligned}
$$

where

$$
\begin{aligned}
& G_{0}^{\prime}\left(\eta^{\prime}\right)=\left|D^{\prime}\right| \\
& G_{n}^{\prime}\left(\eta^{\prime}\right)=D^{\prime}\left[\frac{\left(\eta^{\prime}\right)^{n}}{n !}\left|D^{\prime}\right|^{n-1} D^{\prime}\right]-\sum_{m=0}^{n-1} G_{m}^{\prime}\left(\eta^{\prime}\right) \alpha^{m}\left[\frac{\left(\eta^{\prime}\right)^{n-m}}{(n-m) !}\left|D^{\prime}\right|^{n-m}\right], \quad n \geq 1
\end{aligned}
$$


and

$$
\begin{aligned}
A_{0}^{\prime} & =\frac{1}{2} \\
A_{2 n}^{\prime}\left(\eta^{\prime}\right) & =(-1)^{n} A_{0}^{\prime}\left(\nabla_{x}^{\prime}\left(\eta^{\prime}\right) \cdot \nabla_{x}^{\prime}\left(\eta^{\prime}\right)\right)^{n} \\
A_{2 n+1}^{\prime}\left(\eta^{\prime}\right) & =0
\end{aligned}
$$

and

$$
\begin{aligned}
B_{1}^{\prime}\left(\eta^{\prime}, \xi^{\prime}\right)= & \nabla_{x^{\prime}} \xi^{\prime} \cdot \nabla_{x^{\prime}} \xi^{\prime}-\left(G_{0}^{\prime}\left(\eta^{\prime}\right)\left[\xi^{\prime}\right]\right)^{2} \\
B_{2}^{\prime}\left(\eta^{\prime}, \xi^{\prime}\right)= & -2\left(G_{0}^{\prime}\left(\eta^{\prime}\right)\left[\xi^{\prime}\right]\right) \cdot\left(G_{1}^{\prime}\left(\eta^{\prime}\right)\left[\xi^{\prime}\right]\right)-2 \frac{T}{L}\left(G_{0}^{\prime}\left(\eta^{\prime}\right)\left[\xi^{\prime}\right]\right)\left(\nabla_{x^{\prime}} \eta^{\prime} \cdot \nabla_{x^{\prime}} \eta^{\prime}\right) \\
B_{3}^{\prime}\left(\eta^{\prime}, \xi^{\prime}\right)= & -2\left(G_{0}^{\prime}\left(\eta^{\prime}\right)\left[\xi^{\prime}\right]\right)\left(G_{2}^{\prime}\left(\eta^{\prime}\right)\left[\xi^{\prime}\right]\right)-\left(G_{1}^{\prime}\left(\eta^{\prime}\right)\left[\xi^{\prime}\right]\right)^{2}-2 \frac{T}{L}\left(G_{1}^{\prime}\left(\eta^{\prime}\right)\left[\xi^{\prime}\right]\right)\left(\nabla_{x^{\prime}} \eta^{\prime} \cdot \nabla_{x^{\prime}} \eta^{\prime}\right) \\
& \quad+\left(\nabla_{x^{\prime}} \xi^{\prime} \cdot \nabla_{x^{\prime}} \xi^{\prime}\right)\left(\nabla_{x^{\prime}} \eta^{\prime} \cdot \nabla_{x^{\prime}} \eta^{\prime}\right)-\frac{L^{2}}{T^{2}}\left(\nabla_{x^{\prime}} \xi^{\prime} \cdot \nabla_{x^{\prime}} \xi^{\prime}\right)^{2} \\
B_{n}^{\prime}\left(\eta^{\prime}, \xi^{\prime}\right)= & \sum_{l=0}^{n-1} G_{n-l-1}^{\prime}\left(\eta^{\prime}\right)\left[\xi^{\prime}\right] \cdot G_{l}^{\prime}\left(\eta^{\prime}\right)\left[\xi^{\prime}\right]-2 \cdot \frac{T}{L} G_{n-2}^{\prime}\left(\eta^{\prime}\right)\left[\xi^{\prime}\right]\left(\nabla_{x^{\prime}} \eta^{\prime} \cdot \nabla_{x^{\prime}} \eta^{\prime}\right), \quad n \geq 4
\end{aligned}
$$

Proof. For (Equation 2.6.2):

By definition

$$
G\left(a \eta^{\prime}\right)=\sum_{n=0}^{\infty} G_{n}\left(a \eta^{\prime}\right) .
$$

Clearly $D=\frac{1}{L} D^{\prime}$ by (Equation 2.6.1). From (Equation 3.0.2), we have $G_{0}\left(a \eta^{\prime}\right)=|D|$, thus $G_{0}\left(a \eta^{\prime}\right)=\frac{1}{L} G_{0}^{\prime}\left(\eta^{\prime}\right) \alpha^{0}$, where $G_{0}^{\prime}\left(\eta^{\prime}\right)=\left|D^{\prime}\right|$. 
Now suppose $G_{m}\left(a \eta^{\prime}\right)=\frac{1}{L} G_{m}^{\prime}\left(\eta^{\prime}\right) \alpha^{m}$ for $1 \leq m \leq n-1$. By (Equation 3.0.5) with $f=a \eta^{\prime}$,

$$
\begin{aligned}
G_{n}\left(a \eta^{\prime}\right) & =D\left[F_{n}|D|^{n-1} D\right]-\sum_{m=0}^{n-1} G_{m}\left(a \eta^{\prime}\right)\left[F_{n-m}|D|^{n-m}\right] \\
& =D\left[\frac{\left(a \eta^{\prime}\right)^{n}}{n !}|D|^{n-1} D\right]-\sum_{m=0}^{n-1} \frac{1}{L} G_{m}^{\prime}\left(\eta^{\prime}\right) \alpha^{m}\left[\frac{\left(a \eta^{\prime}\right)^{n-m}}{(n-m) !}|D|^{n-m}\right] \\
& =\frac{1}{L}\left(\frac{a}{L}\right)^{n} D^{\prime}\left[\frac{\left(\eta^{\prime}\right)^{n}}{n !}\left|D^{\prime}\right|^{n-1} D^{\prime}\right]-\frac{1}{L} \alpha^{m}\left(\frac{a}{L}\right)^{n-m} \sum_{m=0}^{n-1} G_{m}^{\prime}\left(\eta^{\prime}\right) \alpha^{m}\left[\frac{\left(\eta^{\prime}\right)^{n-m}}{(n-m) !}\left|D^{\prime}\right|^{n-m}\right] \\
& =\frac{1}{L} \alpha^{n} D^{\prime}\left[\frac{\left(\eta^{\prime}\right)^{n}}{n !}\left|D^{\prime}\right|^{n-1} D^{\prime}\right]-\frac{1}{L} \alpha^{n} \sum_{m=0}^{n-1} G_{m}^{\prime}\left(\eta^{\prime}\right) \alpha^{m}\left[\frac{\left(\eta^{\prime}\right)^{n-m}}{(n-m) !}\left|D^{\prime}\right|^{n-m}\right] \\
& =\frac{1}{L} G_{n}^{\prime}\left(\eta^{\prime}\right) \alpha^{n},
\end{aligned}
$$

and (Equation 2.6.2) is established.

For (Equation 2.6.3):

We first establish some useful relationships for the series for $A(\eta)$. Let $\epsilon>0$ be given and suppose $\eta=\epsilon f$ and

$$
A(\eta)=\sum_{n=0}^{\infty} A_{n}(f) \epsilon^{n}
$$

Using (Equation 2.2.3a), upon cross multiplying we have

$$
2 A(\eta)+2\left(\nabla_{x} \eta \cdot \nabla_{x} \eta\right) A(\eta)=1
$$

Inserting (Equation 2.6.14) into (Equation 2.6.15) and keeping in mind that $\eta=\epsilon f$ yields

$$
2 \sum_{n=0}^{\infty} A_{n}(f) \epsilon^{n}+2 \epsilon^{2}\left(\nabla_{x} f \cdot \nabla_{x} f\right) \sum_{n=0}^{\infty} A_{n}(f) \epsilon^{n}=1
$$


Equating the LHS and RHS of (Equation 2.6.16) immediately yields $A_{0}(f)=\frac{1}{2}$ and $A_{1}(f)=$ 0. Moreover, we easily deduce the relationships:

$$
\begin{aligned}
A_{2 j+1}(f) & =-A_{2 j-1}(f), \quad j \geq 1 \\
A_{2 j}(f) & =(-1)^{j} A_{0}\left(\nabla_{x}(f) \cdot \nabla_{x}(f)\right)^{j}, \quad j \geq 0
\end{aligned}
$$

(Equation 2.6.17) implies $A_{2 j+1}(f)=0$ for all $j \geq 0$. If we let $\epsilon=1, A_{0}^{\prime}=A_{0}$, and consider

$$
A\left(a \eta^{\prime}\right)=\sum_{n=0}^{\infty} A_{n}\left(a \eta^{\prime}\right)
$$

then for all $j \geq 0$, by (Equation 2.6.18) and (Equation 2.6.1)

$$
\begin{aligned}
A_{2 j}\left(a \eta^{\prime}\right) & =(-1)^{j} A_{0}\left(\nabla_{x}\left(a \eta^{\prime}\right) \cdot \nabla_{x}\left(a \eta^{\prime}\right)\right)^{j} \\
& =(-1)^{j} A_{0}^{\prime}\left(\frac{a^{2}}{L^{2}} \nabla_{x}^{\prime}\left(\eta^{\prime}\right) \cdot \nabla_{x}^{\prime}\left(\eta^{\prime}\right)\right)^{j} \\
& =(-1)^{j} A_{0}^{\prime}\left(\alpha^{2} \nabla_{x}^{\prime}\left(\eta^{\prime}\right) \cdot \nabla_{x}^{\prime}\left(\eta^{\prime}\right)\right)^{j} \\
& =(-1)^{j} A_{0}^{\prime}\left(\nabla_{x}^{\prime}\left(\eta^{\prime}\right) \cdot \nabla_{x}^{\prime}\left(\eta^{\prime}\right)\right)^{j} \alpha^{2 j} \\
& =A_{2 j}^{\prime}\left(\eta^{\prime}\right) \alpha^{2 j} .
\end{aligned}
$$

This establishes (Equation 2.6.3).

For (Equation 2.6.4): 
Suppose

$$
B(\eta, \xi)=\sum_{n=0}^{\infty} B_{n}(\eta, \xi) .
$$

Using (Equation 2.2.3b), (Equation 2.6.2), (Equation 2.6.1), Property 5 of Appendix A, and the convolution of sums for the series for $\left.\left(G\left(a \eta^{\prime}\right)\left[X \xi^{\prime}\right]\right)\right)^{2}$ we have

$$
\begin{aligned}
B\left(a \eta^{\prime}, X \xi^{\prime}\right)= & \sum_{n=0}^{\infty} B_{n}\left(a \eta^{\prime}, X \xi^{\prime}\right) \\
= & \frac{X^{2}}{L^{2}}\left(\nabla_{x^{\prime}} \xi^{\prime} \cdot \nabla_{x^{\prime}} \xi^{\prime}\right)-\frac{X^{2}}{L^{2}}\left(\sum_{n=0}^{\infty}\left(\sum_{l=0}^{n} G_{n-l}^{\prime}\left(\eta^{\prime}\right)\left[\xi^{\prime}\right] \cdot G_{l}^{\prime}\left(\eta^{\prime}\right)\left[\xi^{\prime}\right]\right) \alpha^{n}\right) \\
& -2 \cdot \frac{X}{L} \cdot \frac{a^{2}}{L^{2}}\left(\sum_{n=0}^{\infty} G_{n}^{\prime}\left(\eta^{\prime}\right)\left[\xi^{\prime}\right] \alpha^{n}\right)\left(\nabla_{x^{\prime}} \eta^{\prime} \cdot \nabla_{x^{\prime}} \eta^{\prime}\right) \\
& +\frac{X^{2}}{L^{2}} \cdot \frac{a^{2}}{L^{2}}\left(\nabla_{x^{\prime}} \xi^{\prime} \cdot \nabla_{x^{\prime}} \xi^{\prime}\right)\left(\nabla_{x^{\prime}} \eta^{\prime} \cdot \nabla_{x^{\prime}} \eta^{\prime}\right)-\frac{X^{4}}{L^{4}}\left(\nabla_{x^{\prime}} \xi^{\prime} \cdot \nabla_{x^{\prime}} \xi^{\prime}\right)^{2} .
\end{aligned}
$$

Noting $\frac{a^{2}}{L^{2}}=\alpha^{2}$ and $\frac{X}{L}=\frac{a \sqrt{g L}}{L}=\frac{a \sqrt{g}}{\sqrt{L}}=\frac{a}{T}=\frac{\alpha L}{T}$, we have

$$
\begin{aligned}
B\left(a \eta^{\prime}, X \xi^{\prime}\right)=\frac{X}{L} \cdot \frac{L}{T}[ & \left(\nabla_{x^{\prime}} \xi^{\prime} \cdot \nabla_{x^{\prime}} \xi^{\prime}\right) \alpha-\left(\sum_{n=0}^{\infty}\left(\sum_{l=0}^{n} G_{n-l}^{\prime}\left(\eta^{\prime}\right)\left[\xi^{\prime}\right] \cdot G_{l}^{\prime}\left(\eta^{\prime}\right)\left[\xi^{\prime}\right]\right) \alpha^{n+1}\right) \\
& -2 \cdot \frac{T}{L}\left(\sum_{n=0}^{\infty} G_{n}^{\prime}\left(\eta^{\prime}\right)\left[\xi^{\prime}\right] \alpha^{n+2}\right)\left(\nabla_{x^{\prime}} \eta^{\prime} \cdot \nabla_{x^{\prime}} \eta^{\prime}\right) \\
& \left.+\left(\nabla_{x^{\prime}} \xi^{\prime} \cdot \nabla_{x^{\prime}} \xi^{\prime}\right)\left(\nabla_{x^{\prime}} \eta^{\prime} \cdot \nabla_{x^{\prime}} \eta^{\prime}\right) \alpha^{3}-\frac{L^{2}}{T^{2}}\left(\nabla_{x^{\prime}} \xi^{\prime} \cdot \nabla_{x^{\prime}} \xi^{\prime}\right)^{2} \alpha^{3}\right] .
\end{aligned}
$$

From (Equation 2.6.19) we first notice that there are no terms at order $\alpha^{0}$. Therefore, we may write

$$
B\left(a \eta^{\prime}, X \xi^{\prime}\right)=\frac{X}{T} \sum_{n=1}^{\infty} B_{n}^{\prime}\left(\eta^{\prime}, \xi^{\prime}\right) \alpha^{n}
$$


where, picking off the terms at each order we find

$$
\begin{aligned}
& B_{1}^{\prime}\left(\eta^{\prime}, \xi^{\prime}\right)= \nabla_{x^{\prime}} \xi^{\prime} \cdot \nabla_{x^{\prime}} \xi^{\prime}-\left(G_{0}^{\prime}\left(\eta^{\prime}\right)\left[\xi^{\prime}\right]\right)^{2} \\
& B_{2}^{\prime}\left(\eta^{\prime}, \xi^{\prime}\right)=-2\left(G_{0}^{\prime}\left(\eta^{\prime}\right)\left[\xi^{\prime}\right]\right) \cdot\left(G_{1}^{\prime}\left(\eta^{\prime}\right)\left[\xi^{\prime}\right]\right)-2 \frac{T}{L}\left(G_{0}^{\prime}\left(\eta^{\prime}\right)\left[\xi^{\prime}\right]\right)\left(\nabla_{x^{\prime}} \eta^{\prime} \cdot \nabla_{x^{\prime}} \eta^{\prime}\right) \\
& B_{3}^{\prime}\left(\eta^{\prime}, \xi^{\prime}\right)=-2\left(G_{0}^{\prime}\left(\eta^{\prime}\right)\left[\xi^{\prime}\right]\right)\left(G_{2}^{\prime}\left(\eta^{\prime}\right)\left[\xi^{\prime}\right]\right)-\left(G_{1}^{\prime}\left(\eta^{\prime}\right)\left[\xi^{\prime}\right]\right)^{2}-2 \frac{T}{L}\left(G_{1}^{\prime}\left(\eta^{\prime}\right)\left[\xi^{\prime}\right]\right)\left(\nabla_{x^{\prime}} \eta^{\prime} \cdot \nabla_{x^{\prime}} \eta^{\prime}\right) \\
& \quad+\left(\nabla_{x^{\prime}} \xi^{\prime} \cdot \nabla_{x^{\prime}} \xi^{\prime}\right)\left(\nabla_{x^{\prime}} \eta^{\prime} \cdot \nabla_{x^{\prime}} \eta^{\prime}\right)-\frac{L^{2}}{T^{2}}\left(\nabla_{x^{\prime}} \xi^{\prime} \cdot \nabla_{x^{\prime}} \xi^{\prime}\right)^{2} .
\end{aligned}
$$

For $n \geq 4$, reindexing the series in (Equation 2.6.19) helps to yield a compact formula for each $n$. Thus if we now examine

$$
-\sum_{n=1}^{\infty}\left(\sum_{l=0}^{n-1} G_{n-l-1}^{\prime}\left(\eta^{\prime}\right)\left[\xi^{\prime}\right] \cdot G_{l}^{\prime}\left(\eta^{\prime}\right)\left[\xi^{\prime}\right]\right) \alpha^{n}-2 \cdot \frac{T}{L}\left(\sum_{n=2}^{\infty} G_{n-2}^{\prime}\left(\eta^{\prime}\right)\left[\xi^{\prime}\right] \alpha^{n}\right)\left(\nabla_{x^{\prime}} \eta^{\prime} \cdot \nabla_{x^{\prime}} \eta^{\prime}\right)
$$

we see that for $n \geq 4$

$$
B_{n}^{\prime}\left(\eta^{\prime}, \xi^{\prime}\right)=\sum_{l=0}^{n-1} G_{n-l-1}^{\prime}\left(\eta^{\prime}\right)\left[\xi^{\prime}\right] \cdot G_{l}^{\prime}\left(\eta^{\prime}\right)\left[\xi^{\prime}\right]-2 \cdot \frac{T}{L} G_{n-2}^{\prime}\left(\eta^{\prime}\right)\left[\xi^{\prime}\right]\left(\nabla_{x^{\prime}} \eta^{\prime} \cdot \nabla_{x^{\prime}} \eta^{\prime}\right)
$$


We now show that (Equation 2.5.3) becomes

$$
\begin{aligned}
& \partial_{t^{\prime}} \eta^{\prime}+c^{\prime} \cdot \nabla_{x^{\prime}} \eta^{\prime}=G_{0}^{\prime}\left(\eta^{\prime}\right)\left[\xi^{\prime}\right]+\sum_{n=1}^{\infty} G_{n}^{\prime}\left(\eta^{\prime}\right)\left[\xi^{\prime}\right] \alpha^{n} \\
& \partial_{t^{\prime}} \xi^{\prime}+c^{\prime} \cdot \nabla_{x^{\prime}} \xi^{\prime}=-\eta^{\prime}-\sum_{n=1}^{\infty} \alpha^{n}\left(\sum_{l=1}^{n} A_{n-l}^{\prime}\left(\eta^{\prime}\right) B_{l}^{\prime}\left(\eta^{\prime}, \xi^{\prime}\right)\right)
\end{aligned}
$$

where we have defined the dimensionless velocity as

$$
c^{\prime}=\frac{c}{\sqrt{g L}}
$$

which in two dimensions is the Froude number.

Using Definition 2.6.1, (Equation 2.6.1), Proposition 2.6.1, and Property 1 from Appendix A, (Equation 2.5.3a) becomes

$$
\frac{a}{T} \partial_{t^{\prime}} \eta^{\prime}+\frac{a c}{L} \nabla_{x^{\prime}} \eta^{\prime}=\frac{X}{L} \sum_{n=0}^{\infty} G_{n}^{\prime}\left(\eta^{\prime}\right)\left[\xi^{\prime}\right] \alpha^{n}
$$

Multiplying both sides of this equation by $\frac{L}{X}$ and noting $\frac{L}{X} \cdot \frac{a}{T}=1$ and $\frac{c a}{X}=\frac{c}{\sqrt{g L}}$, establishes (Equation 2.6.20).

Using Definition 2.6.1, (Equation 2.6.1), Proposition 2.6.1, and the convolution of sums, (Equation 2.5.3b) becomes

$$
\frac{X}{T} \partial_{t^{\prime}} \xi^{\prime}+\frac{c X}{L} \cdot \nabla_{x^{\prime}} \xi^{\prime}=-g a \eta^{\prime}-\frac{X}{T} \sum_{n=1}^{\infty} \alpha^{n}\left(\sum_{l=1}^{n} A_{n-l}\left(\eta^{\prime}\right) B_{l}\left(\eta^{\prime}, \xi^{\prime}\right)\right)
$$


Multiplying both sides of this equation by $\frac{T}{X}$ and noting $\frac{T C}{L}=c^{\prime}$ and $\frac{T g a}{X}=1$, establishes (Equation 2.6.21).

Finally, we eliminate the explicit dependence upon the (small) dimensionless parameter $\alpha$ by choosing as unknowns the dimensionless quantities

$$
\begin{aligned}
& \tilde{\eta}(x, t):=\alpha \eta^{\prime}(x, t)=\frac{\alpha}{a} \eta(x, t)=\frac{1}{L} \eta(x, t), \\
& \tilde{\xi}(x, t):=\alpha \xi^{\prime}(x, t)=\frac{\alpha}{X} \xi(x, t)=\frac{1}{\sqrt{L^{3} g}} \xi(x, t) .
\end{aligned}
$$

Upon dropping primes and tildes, we find the final dimensionless evolution equations in a traveling frame

$$
\begin{aligned}
& \partial_{t} \eta+c \cdot \nabla_{x} \eta=G(\eta)[\xi] \\
& \partial_{t} \xi+c \cdot \nabla_{x} \xi=-\eta-A(\eta) B(\eta, \xi),
\end{aligned}
$$

periodic on the lattice

$$
\Gamma_{\theta}=\left\{\gamma=\left(\begin{array}{c}
2 \pi m_{1} / \sin (\theta) \\
2 \pi m_{2} / \cos (\theta)
\end{array}\right) ; m_{1}, m_{2} \in \mathbf{Z}\right\}
$$

with conjugate lattice

$$
\Gamma_{\theta}^{\prime}=\left\{k=\left(\begin{array}{c}
m_{1} \sin (\theta) \\
m_{2} \cos (\theta)
\end{array}\right) ; m_{1}, m_{2} \in \mathbf{Z}\right\},
$$

c.f., (Equation 2.1.2a) \& (Equation 2.1.2b). 


\section{7 $\quad$ Spectral Stability Analysis}

The spectral stability analysis we have in mind, fully described in (26), is summarized here. Consider a steady traveling wave solution of (Equation 2.2.2), that is, a steady solution of (Equation 2.6.22)

$$
(\bar{\eta}, \bar{\xi}, \bar{c})=(\bar{\eta}(x), \bar{\xi}(x), \bar{c})
$$

We seek solutions of the full problem (Equation 2.6.22) in the spectral stability form

$$
\eta(x, t)=\bar{\eta}(x)+\delta e^{\lambda t} \zeta(x), \quad \xi(x, t)=\bar{\xi}(x)+\delta e^{\lambda t} \psi(x),
$$

where $\delta \ll 1$ measures the perturbation from the steady state, and $\lambda$ determines the spectral stability. Inserting this into (Equation 2.6.22) and using the fact that $(\bar{\eta}, \bar{\xi}, \bar{c})$ are solutions of (Equation 2.6.22), we recover, to order $\mathcal{O}(\delta)$,

$$
\begin{aligned}
& \left(\lambda+c \cdot \nabla_{x}\right) \zeta=G_{\eta}(\bar{\eta})[\bar{\xi}]\{\zeta\}+G(\bar{\eta})[\psi] \\
& \left(\lambda+c \cdot \nabla_{x}\right) \psi=-\zeta-A_{\eta}(\bar{\eta})\{\zeta\} B(\bar{\eta}, \bar{\xi})-A(\bar{\eta}) B_{\eta}(\bar{\eta}, \bar{\xi})\{\zeta\}-A(\bar{\eta}) B_{\xi}(\bar{\eta}, \bar{\xi})\{\psi\},
\end{aligned}
$$

where $\eta$ and $\xi$ subscripts denote first variations as described in Propositions 2.3.1 and 3.0.1. 


\section{$2.8 \quad$ Bloch Theory}

We now write our spectral stability problem (Equation 2.7.1) abstractly as

$$
\mathcal{A}(x)\left(\begin{array}{l}
\zeta \\
\psi
\end{array}\right)=\lambda\left(\begin{array}{l}
\zeta \\
\psi
\end{array}\right)
$$

The final specification is the boundary conditions that the eigenfunctions $(\zeta, \psi)$ must satisfy. For these we use the "Generalized Principle of Reduced Instability" (27) (essentially Floquet Theory, see, e.g., (28)), inspired by the Bloch theory of Schrödinger equations with periodic potentials (29). This theory allows perturbations

$$
(\zeta, \psi) \in H_{l u}^{2}\left(\mathbf{R}^{2}\right)
$$

which are in the Sobolev class of uniformly local $L^{2}$ functions. Mielke reduces this study to the "Bloch waves," e.g.,

$$
\zeta(x)=e^{i p \cdot x} Z(x), \quad \psi(x)=e^{i p \cdot x} Y(x)
$$

where $Z, Y \in H^{2}(P(\Gamma))$. As we shall see, it suffices to consider $p \in P\left(\Gamma^{\prime}\right)$, the fundamental cell of wavenumbers, and thus (Equation 2.8.1) becomes

$$
\mathcal{A}_{p}(x)\left(\begin{array}{l}
\zeta \\
\psi
\end{array}\right)=\lambda\left(\begin{array}{l}
\zeta \\
\psi
\end{array}\right)
$$


where

$$
\mathcal{A}_{p}(x)\left(\begin{array}{l}
\zeta \\
\psi
\end{array}\right):=e^{-i p \cdot x} \mathcal{A}(x)\left[e^{i p \cdot x}\left(\begin{array}{l}
\zeta \\
\psi
\end{array}\right)\right]
$$

Mielke's fundamental result (27) is that

$$
L^{2}-\operatorname{spec}(\mathcal{A})=L_{l u}^{2}-\operatorname{spec}(\mathcal{A})=\operatorname{closure}\left(\bigcup_{p \in P\left(\Gamma^{\prime}\right)} \operatorname{spec}\left(\mathcal{A}_{p}\right)\right)
$$

Thus, we learn about stability with respect to all of these perturbations by simply considering $Z, Y \in H^{2}(P(\Gamma))$ and $p \in P\left(\Gamma^{\prime}\right)$. This whole analysis is equivalent to requiring that the functions $\zeta$ and $\psi$ satsify the "Bloch boundary conditions":

$$
\zeta(x+\gamma)=e^{i p \cdot \gamma} \zeta(x), \quad \psi(x+\gamma)=e^{i p \cdot \gamma} \psi(x), \quad \forall \gamma \in \Gamma
$$

Such functions can be expanded in the "generalized" Fourier series

$$
\zeta(x)=\sum_{k \in \Gamma^{\prime}} \hat{\zeta}_{k} e^{i(k+p) \cdot x}, \quad \psi(x)=\sum_{k \in \Gamma^{\prime}} \hat{\psi}_{k} e^{i(k+p) \cdot x},
$$

c.f. (Equation 2.1.1). 


\section{CHAPTER 3}

\section{THE DIRICHLET-NEUMANN OPERATOR AND ITS FIRST VARIATION}

We choose the Method of Operator Expansions $(\mathrm{OE})(30 ; 24 ; 12 ; 31)$ in the case of water of infinite depth to compute the DNO and its first variation. One could choose from other methods such as finite difference, finite element, finite volume, and integral equations.

The aspect of the OE methodology that is the crux for our approach is that for sufficiently smooth deformations $\eta=\varepsilon f$, the DNO and its first variation depend analytically (32) upon the height/slope parameter $\varepsilon \in \mathbf{R}$, resulting in strongly convergent expansions of the form

$$
G(\varepsilon f)=\sum_{n=0}^{\infty} G_{n}(f) \varepsilon^{n}, \quad G_{\eta}(\varepsilon f)=\sum_{n=0}^{\infty} G_{n}^{(1)}(f) \varepsilon^{n}
$$

for $\varepsilon$ sufficiently small and positive.

The OE method yields accurate formulas for the $G_{n}$ and the first variations $G_{n}^{(1)}$ in terms of Fourier multipliers and convolutions of series. To begin our discussion, consider the function

$$
v_{k}(x, y)=e^{i k \cdot x+|k| y}, \quad k \in \Gamma^{\prime}
$$


It satisfies (Equation 2.0.1a) and (Equation 2.0.1b) of the elliptic equations governing the definition of the DNO since

$$
\Delta_{x} v_{k}(x, y)=-|k|^{2} e^{i k \cdot x+|k| y}=-\partial_{y y} v_{k}(x, y)
$$

and

$$
\lim _{y \rightarrow-\infty}|k| e^{i k \cdot x+|k| y}=0 .
$$

Using the definition of the DNO (Equation 2.2.1) with $y=\eta=\epsilon f$ we have

$$
G(\varepsilon f)\left[e^{i k \cdot x+|k| \varepsilon f}\right]=\left(|k|-\varepsilon\left(\nabla_{x} f\right) \cdot(i k)\right) e^{i k \cdot x+|k| \varepsilon f} .
$$

Expanding the DNO and exponentials on the LHS and RHS in power series yields

$$
\left(\sum_{n=0}^{\infty} G_{n}(f) \varepsilon^{n}\right)\left[\sum_{m=0}^{\infty} F_{m}|k|^{m} \varepsilon^{m} e^{i k \cdot x}\right]=\left(|k|-\varepsilon\left(\nabla_{x} f\right) \cdot(i k)\right) \sum_{n=0}^{\infty} F_{n}|k|^{n} \varepsilon^{n} e^{i k \cdot x},
$$

where $F_{n}(x):=\frac{(f(x))^{n}}{n !}$. At order $\mathcal{O}\left(\varepsilon^{0}\right)$ this gives

$$
G_{0}(f)\left[e^{i k \cdot x}\right]=|k| e^{i k \cdot x}=|D|\left[e^{i k \cdot x}\right]
$$


which defines the order-one Fourier multiplier $|D|$ in terms of $D=(1 / i) \partial_{x}$. Noting that any $L^{2}$ function $\xi$ can be written in terms of its Fourier series

$$
\xi(x)=\sum_{k \in \Gamma^{\prime}} \hat{\xi}_{k} e^{i k \cdot x}
$$

we have the action of $G_{0}$ on any $\xi$ given by

$$
G_{0}(f)[\xi]=G_{0}(f)\left[\sum_{k \in \Gamma^{\prime}} \hat{\xi}_{k} e^{i k \cdot x}\right]=\sum_{k \in \Gamma^{\prime}} \hat{\xi}_{k} G_{0}(f)\left[e^{i k \cdot x}\right]=\sum_{k \in \Gamma^{\prime}} \hat{\xi}_{k}|k| e^{i k \cdot x}=:|D|[\xi]
$$

At order $\mathcal{O}\left(\varepsilon^{1}\right)$, equating the LHS and RHS of (Equation 3.0.1) and solving for $G_{1}(f)\left[e^{i k \cdot x}\right]$ yields

$$
G_{1}(f)\left[e^{i k \cdot x}\right]=F_{1}|k|^{2} e^{i k \cdot x}-F_{0}\left(\nabla_{x} f\right) \cdot(i k) e^{i k \cdot x}-G_{0}(f)\left[F_{1}|k|^{1} e^{i k \cdot x}\right] .
$$

In a similar fashion, at order $\mathcal{O}\left(\varepsilon^{2}\right)$ we find

$$
G_{2}(f)\left[e^{i k \cdot x}\right]=F_{2}|k|^{3} e^{i k \cdot x}-F_{1}\left(\nabla_{x} f\right) \cdot(i k)|k|^{1} e^{i k \cdot x}-G_{0}(f)\left[F_{1}|k|^{1} e^{i k \cdot x}\right]-G_{1}(f)\left[F_{0} e^{i k \cdot x}\right]
$$

Thus at order $\mathcal{O}\left(\varepsilon^{n}\right)$ for $n>0$ in (Equation 3.0.1) we deduce the general formula for $G_{n}(f)\left[e^{i k \cdot x}\right]$ and then use the definition of $|D|$, the fact $|D|^{2}=D$, and the product rule for $D$ to write it in a compact form: 


$$
\begin{aligned}
G_{n}(f)\left[e^{i k \cdot x}\right] & =F_{n}|k|^{n+1} e^{i k \cdot x}-F_{n-1}\left(\nabla_{x} f\right) \cdot(i k)|k|^{n-1} e^{i k \cdot x}-\sum_{m=0}^{n-1} G_{m}(f)\left[F_{n-m}|k|^{n-m} e^{i k \cdot x}\right] \\
& =\left(F_{n}|D|^{n+1}+(D f) F_{n-1}|D|^{n-1}\right)\left[e^{i k \cdot x}\right]-\sum_{m=0}^{n-1} G_{m}(f)\left[F_{n-m}|k|^{n-m} e^{i k \cdot x}\right] \\
& =\left(F_{n}|D|^{2}+(D f) F_{n-1} D\right)\left[|D|^{n-1}\left[e^{i k \cdot x}\right]\right]-\sum_{m=0}^{n-1} G_{m}(f)\left[F_{n-m}|D|^{n-m} e^{i k \cdot x}\right] \\
& =D\left[F_{n}|D|^{n-1} D\left[e^{i k \cdot x}\right]\right]-\sum_{m=0}^{n-1} G_{m}(f)\left[F_{n-m}|D|^{n-m}\left[e^{i k \cdot x}\right]\right] .
\end{aligned}
$$

Using the Fourier representation of $\xi$ and (Equation 3.0.4) we find

$$
\begin{aligned}
G_{n}(f)[\xi] & =G_{n}(f)\left[\sum_{k \in \Gamma^{\prime}} \hat{\xi}_{k} e^{i k \cdot x}\right] \\
& =\sum_{k \in \Gamma^{\prime}} \hat{\xi}_{k} G_{n}(f)\left[e^{i k \cdot x}\right] \\
& =\sum_{k \in \Gamma^{\prime}} \hat{\xi}_{k}\left(D\left[F_{n}|D|^{n-1} D\left[e^{i k \cdot x}\right]\right]-\sum_{m=0}^{n-1} G_{m}(f)\left[F_{n-m}|D|^{n-m}\left[e^{i k \cdot x}\right]\right]\right) \\
& =D\left[F_{n}|D|^{n-1} D[\xi]\right]-\sum_{m=0}^{n-1} G_{m}(f)\left[F_{n-m}|D|^{n-m}[\xi]\right] .
\end{aligned}
$$

With (Equation 3.0.3) for $G_{0}$ and (Equation 3.0.5) for $G_{n}$ we derive the first variation of the DNO and its perturbation expansion $(12 ; 33 ; 34)$. For this derivation it is helpful to think of the expansion of the DNO (equivalently) as

$$
G(\eta)=\sum_{n=0}^{\infty} G_{n}(\eta)
$$


$\left(G_{0}\right.$ and $G_{n}$ are given in (Equation 3.0.3) \& (Equation 3.0.5), respectively, with $f$ replaced by $\eta)$. Taking the variation with respect to $\eta$ we find

$$
G_{\eta}(\eta)\{\zeta\}=\left(\delta_{\eta} G\right)(\eta)\{\zeta\}=\sum_{n=1}^{\infty}\left(\delta_{\eta} G_{n}\right)(\eta)\{\zeta\}
$$

where the $n=0$ term disappears since $G_{0}(\eta)[\xi]$ is independent of $\eta$. The first variation of $G$ is an important component of our spectral stability analysis.

Proposition 3.0.1. For $n>0, n \in \mathbb{N}$

$$
\begin{aligned}
\left(\delta_{\eta} G_{n}(\eta)\right)[\xi]\{\zeta\}= & D\left[\zeta\left(\frac{\eta^{n-1}}{(n-1) !}\right)|D|^{n-1} D[\xi]\right]-\sum_{m=0}^{n-1}\left(\delta_{\eta} G_{m}(\eta)\right)\left[\left(\frac{\eta^{n-m}}{(n-m) !}\right)|D|^{n-m}[\xi]\right]\{\zeta\} \\
& -\sum_{m=0}^{n-1} G_{m}(\eta)\left[\zeta\left(\frac{\eta^{n-m-1}}{(n-m-1) !}\right)|D|^{n-m}[\xi]\right]
\end{aligned}
$$

Proof. Given $n>0$, by definition

$$
\left(\delta_{\eta} G_{n}(\eta)\right)[\xi]\{\zeta\}=\lim _{\epsilon \rightarrow 0} \frac{G_{n}(\eta+\epsilon \zeta)[\xi]-G_{n}(\eta)[\xi]}{\epsilon} .
$$


Noting the definition of $F_{n}$ from (Equation 3.0.1), using (Equation 3.0.5), and properties of the DNO in Appendix A, we first work out the numerator:

$$
\begin{aligned}
G_{n}(\eta+\epsilon \zeta)[\xi]-G_{n}(\eta)[\xi]= & D\left[\frac{(\eta+\epsilon \zeta)^{n}}{n !}|D|^{n-1} D[\xi]\right]-\sum_{m=0}^{n-1} G_{m}(\eta+\epsilon \zeta)\left[\frac{(\eta+\epsilon \zeta)^{n-m}}{(n-m) !}|D|^{n-m}[\xi]\right] \\
& -D\left[\frac{\eta^{n}}{n !}|D|^{n-1} D[\xi]\right]+\sum_{m=0}^{n-1} G_{m}(\eta)\left[\frac{\eta^{n-m}}{(n-m) !}|D|^{n-m}[\xi]\right] .
\end{aligned}
$$

We break this down by first noting

$$
\begin{aligned}
D\left[\frac{(\eta+\epsilon \zeta)^{n}}{n !}|D|^{n-1} D[\xi]\right]= & D\left[\frac{\eta^{n}}{n !}|D|^{n-1} D[\xi]\right]+D\left[\sum_{k=1}^{n}\left(\begin{array}{l}
n \\
k
\end{array}\right) \frac{\eta^{n-k}}{n !} \epsilon^{k} \zeta^{k}|D|^{n-1} D[\xi]\right] \\
= & D\left[\frac{\eta^{n}}{n !}|D|^{n-1} D[\xi]\right]+D\left[\sum_{k=1}^{n} \frac{\eta^{n-k}}{k !(n-k) !} \epsilon^{k} \zeta^{k}|D|^{n-1} D[\xi]\right] \\
= & D\left[\frac{\eta^{n}}{n !}|D|^{n-1} D[\xi]\right]+\epsilon D\left[\frac{\eta^{n-1}}{(n-1) !}|D|^{n-1} D[\xi]\right] \\
& +\sum_{k=2}^{n} D\left[\frac{\eta^{n-k}}{k !(n-k) !} \zeta^{k}|D|^{n-1} D[\xi]\right] \epsilon^{k} .
\end{aligned}
$$


Next, we see that

$$
\begin{gathered}
-\sum_{m=0}^{n-1} G_{m}(\eta+\epsilon \zeta)\left[\frac{(\eta+\epsilon \zeta)^{n-m}}{(n-m) !}|D|^{n-m}[\xi]\right]+\sum_{m=0}^{n-1} G_{m}(\eta)\left[\frac{\eta^{n-m}}{(n-m) !}|D|^{n-m}[\xi]\right] \\
=-\sum_{m=0}^{n-1} G_{m}(\eta+\epsilon \zeta)\left[\frac{\eta^{n-m}}{(n-m) !}|D|^{n-m}[\xi]\right]-\sum_{m=0}^{n-1} G_{m}(\eta+\epsilon \zeta)\left[\sum_{k=1}^{n-m}\left(\begin{array}{c}
n-m \\
k
\end{array}\right) \frac{\eta^{n-m-k}}{(n-m) !} \epsilon^{k} \zeta^{k}|D|^{n-m}[\xi]\right] \\
\quad+\sum_{m=0}^{n-1} G_{m}(\eta)\left[\frac{\eta^{n-m}}{(n-m) !}|D|^{n-m}[\xi]\right] \\
=-\sum_{m=0}^{n-1}\left\{G_{m}(\eta+\epsilon \zeta)\left[\frac{\eta^{n-m}}{(n-m) !}|D|^{n-m}[\xi]\right]-G_{m}(\eta)\left[\frac{\eta^{n-m}}{(n-m) !}|D|^{n-m}[\xi]\right]\right\} \\
-\sum_{m=0}^{n-1} G_{m}(\eta+\epsilon \zeta)\left[\sum_{k=1}^{n-m} \frac{\eta^{n-m-k}}{k !(n-m-k) !} \epsilon^{k} \zeta^{k}|D|^{n-m}[\xi]\right] \\
=-\sum_{m=0}^{n-1}\left\{G_{m}(\eta+\epsilon \zeta)\left[\frac{\eta^{n-m}}{(n-m) !}|D|^{n-m}[\xi]\right]-G_{m}(\eta)\left[\frac{\eta^{n-m}}{(n-m) !}|D|^{n-m}[\xi]\right]\right\} \\
\quad-\epsilon \sum_{m=0}^{n-1} G_{m}(\eta+\epsilon \zeta)\left[\frac{\eta^{n-m-1}}{(n-m-1) !} \zeta|D|^{n-m}[\xi]\right] \\
\quad \sum_{m=0}^{n-1} \sum_{k=2}^{n-m} G_{m}(\eta+\epsilon \zeta)\left[\frac{\eta^{n-m-k}}{k !(n-m-k) !} \zeta^{k}|D|^{n-m}[\xi]\right] \epsilon^{k} . \quad(3.0 .10)
\end{gathered}
$$


Inserting (Equation 3.0.9) and (Equation 3.0.10) into (Equation 3.0.8) and applying the result to (Equation 3.0.7) yields

$$
\begin{gathered}
\left(\delta_{\eta} G_{n}(\eta)\right)[\xi]\{\zeta\}=\lim _{\epsilon \rightarrow 0}\left(D\left[\frac{\eta^{n-1}}{(n-1) !}|D|^{n-1} D[\xi]\right]+\sum_{k=2}^{n} D\left[\frac{\eta^{n-k}}{k !(n-k) !} \zeta^{k}|D|^{n-1} D[\xi]\right] \epsilon^{k-1}\right. \\
-\sum_{m=0}^{n-1}\left\{\frac{G_{m}(\eta+\epsilon \zeta)\left[\frac{\eta^{n-m}}{(n-m) !}|D|^{n-m}[\xi]\right]-G_{m}(\eta)\left[\frac{\eta^{n-m}}{(n-m) !}|D|^{n-m}[\xi]\right]}{\epsilon}\right\} \\
\left.-\sum_{m=0}^{n-1} G_{m}(\eta+\epsilon \zeta)\left[\frac{\eta^{n-m-1}}{(n-m-1) !} \zeta|D|^{n-m}[\xi]\right]-\sum_{m=0}^{n-1} \sum_{k=2}^{n-m} G_{m}(\eta+\epsilon \zeta)\left[\frac{\eta^{n-m-k}}{k !(n-m-k) !} \zeta^{k}|D|^{n-m}[\xi]\right] \epsilon^{k-1}\right) \\
=D\left[\zeta\left(\frac{\eta^{n-1}}{(n-1) !}\right)|D|^{n-1} D[\xi]\right]-\sum_{m=0}^{n-1}\left(\delta_{\eta} G_{m}(\eta)\right)\left[\left(\frac{\eta^{n-m}}{(n-m) !}\right)|D|^{n-m}[\xi]\right]\{\zeta\} \\
-\sum_{m=0}^{n-1} G_{m}(\eta)\left[\zeta\left(\frac{\eta^{n-m-1}}{(n-m-1) !}\right)|D|^{n-m}[\xi]\right]
\end{gathered}
$$

as claimed in (Equation 3.0.6).

In an expansion in powers of $\varepsilon$ with $\eta=\varepsilon f$, clearly the $n$th order term is only $\mathcal{O}\left(\varepsilon^{n-1}\right)$, where

$$
\begin{aligned}
G_{n}^{(1)}(f)[\xi]\{\zeta\}= & D\left[\zeta F_{n-1}|D|^{n-1} D[\xi]\right] \\
& -\sum_{m=0}^{n-1} G_{m}^{(1)}(f)\left[F_{n-m}|D|^{n-m}[\xi]\right]\{\zeta\} \\
& -\sum_{m=0}^{n-1} G_{m}(f)\left[\zeta F_{n-m-1}|D|^{n-m}[\xi]\right] .
\end{aligned}
$$




\section{CHAPTER 4}

\section{LINEAR STABILITY THEORY}

To properly frame our discussion of the spectral stability theory of nonlinear traveling water waves, we must first discuss the linear theory. The traveling waves we study bifurcate from the trivial state

$$
(\bar{\eta}, \bar{\xi}, \bar{c})=\left(0,0, c_{0}\right)
$$

for values of the velocity $\bar{c}=c_{0}$ discussed below.

The order zero stability result involves the study of these forms in the spectral stability problem (Equation 2.7.1). We now find the order zero form of this problem.

For $\epsilon \ll 1$, we expand

$$
\zeta(x)=\sum_{n=0}^{\infty} \zeta_{n}(x) \epsilon^{n}, \quad \psi(x)=\sum_{n=0}^{\infty} \psi_{n}(x) \epsilon^{n} .
$$

For the LHS of (Equation 2.7.1a), clearly the only term of order zero that contributes is $\zeta_{0}$. For the RHS of (Equation 2.7.1a), from Proposition 3.0.1 we know that $G_{\eta}(\bar{\eta})[\bar{\xi}]\{\zeta\}$ contains no terms at order zero, while if we use the series expansion for $G(\bar{\eta})[\psi]$, we pick up $G_{0}(\bar{\eta})[\psi]$.

For the LHS of (Equation 2.7.1b), clearly the only order zero contribution is $\psi_{0}$. For the RHS of (Equation 2.7.1b), we first pick off $-\zeta_{0}$. None of the remaining terms make a contribution

given the expressions for $A_{\eta}(\zeta)\{\bar{\eta}\}$ and $A(\bar{\eta})$ from Propositions 2.3.1 and 2.2.1, respectively. 
Replacing $\bar{c}$ with $c_{0}$, we now have the order zero form of (Equation 2.7.1):

$$
\begin{aligned}
& \left(\lambda+c_{0} \cdot \nabla_{x}\right) \zeta_{0}=G_{0}\left[\psi_{0}\right] \\
& \left(\lambda+c_{0} \cdot \nabla_{x}\right) \psi_{0}=-\zeta_{0},
\end{aligned}
$$

or

$$
\left(\begin{array}{cc}
\lambda_{0}+c_{0} \cdot \nabla_{x} & -G_{0} \\
1 & \lambda_{0}+c_{0} \cdot \nabla_{x}
\end{array}\right)\left(\begin{array}{l}
\zeta_{0} \\
\psi_{0}
\end{array}\right)=\mathbf{0}
$$

Expressing $\zeta_{0}$ and $\psi_{0}$ in terms of their generalized Fourier series

$$
\zeta_{0}(x)=\sum_{k \in \Gamma^{\prime}} \hat{\zeta}_{0, k} e^{i(k+p) \cdot x}, \quad \psi_{0}(x)=\sum_{k \in \Gamma^{\prime}} \hat{\psi}_{0, k} e^{i(k+p) \cdot x}
$$

and recalling that $G_{0}=|D|$, this reduces to

$$
\left(\begin{array}{cc}
\lambda_{0}+c_{0} \cdot(i(k+p)) & -|k+p| \\
1 & \lambda_{0}+c_{0} \cdot(i(k+p))
\end{array}\right)\left(\begin{array}{c}
\hat{\zeta}_{0, k} \\
\hat{\psi}_{0, k}
\end{array}\right)=\mathbf{0} .
$$

Given a velocity $c_{0}$, and the quasiperiodicity parameter $p$, we find nontrivial solutions for all $k \in \Gamma^{\prime}$ for two choices of the eigenvalue $\lambda_{0}$ which make the determinant function

$$
\Lambda\left(\lambda_{0}, c_{0}, k, p\right):=\left(\lambda_{0}+i c_{0} \cdot(k+p)\right)^{2}+|k+p|
$$


equal to zero. Using the quadratic formula to solve for $\lambda_{0}$, and a little algebra yield

$$
\lambda_{0}^{s}(k, p)=i\left(-c_{0} \cdot(k+p)+s \sqrt{|k+p|}\right)=i\left(-c_{0} \cdot(k+p)+s \sqrt{\omega_{k+p}}\right)
$$

where $s= \pm 1$ and $\omega_{k}:=\sqrt{|k|}$ is the dispersion relation. These eigenvalues are purely imaginary, which indicates that the trivial, flat water state is weakly stable: Small perturbations will not grow, but neither will they decay.

Our primary interest is in what happens as we move from trivial (zero-amplitude) traveling waves to non-trivial (finite-amplitude) waves. The spectrum will "move" as the wave amplitude, for instance, is varied from zero and the natural question arises: Does one or more of the eigenvalues move into the complex right half-plane, that is, do we get $\operatorname{Re}\left\{\lambda_{0}^{s}(k, p)\right\}>0$ for any eigenvalue?

The results of MacKay \& Saffman (35), which extend well-known results for finite dimensional Hamiltonian systems to the water wave problem, give us a necessary but not sufficient (36) condition for such an excursion for an eigenvalue: This may only occur after "collision" with another eigenvalue. More precisely, only after an eigenvalue increases its multiplicity larger than one.

To detect the onset of instability, meaning the smallest amplitude traveling wave for which we achieve $\operatorname{Re}\left\{\lambda_{0}^{s}(k, p)\right\}>0$, it is natural to focus upon eigenvalues of higher multiplicity among the trivial wave spectrum of (Equation 4.0.2). If these eigenvalues are distinct (multiplicity one) for a particular configuration (e.g., a fixed value of $p \in P\left(\Gamma^{\prime}\right)$ ), then there is guaranteed 
a small "window of stability" for small enough amplitudes $(26 ; 31)$. However, if one of these is multiplicity two or higher, there may be "immediate" instability, which means instability for all amplitudes larger than zero.

The work of Ioualalen \& Kharif $(15 ; 16)$ focuses precisely on these "resonant configurations," i.e., values of $p \in P\left(\Gamma^{\prime}\right)$ where eigenvalues of multiplicity two or higher exist. These values of $p$ can be characterized by the condition

$$
\lambda_{0}^{s_{1}}\left(k_{1}, p\right)=\lambda_{0}^{s_{2}}\left(k_{2}, p\right), \quad k_{1} \neq k_{2},
$$

and it can be shown that $s_{2}$ must be the opposite of $s_{1}$.

Following McLean's work on the three-dimensional stability of Stokes waves $(37 ; 38 ; 39)$, Ioualalen \& Kharif define assorted classes of resonances based upon the parity of the components of the difference $k_{1}-k_{2}$, and then plot the resulting curves in $p$-space for these classes. For SCWs they then indicate, for specified values of the traveling wave amplitude, which values of $p$ give rise to spectrum with positive real part. While the results are not detailed, for example they do not give quantitative information on the magnitude of the largest real part, they do show that the lowest-order $\left(k_{1}-k_{2}\right.$ small $)$ resonances are dominant for small amplitudes. We will replicate this analysis for GSCWs. 


\section{CHAPTER 5}

\section{GENERALIZED SHORT CRESTED WAVES}

Having discussed the stability of flat water, we now narrow down to our primary focus. To begin, we will define these GSCWs by recalling the governing equations for water waves in a reference frame traveling with velocity $\bar{c}$ (Equation 2.6.22), and focus on steady, linear solutions which satisfy

$$
\begin{aligned}
& \bar{c}_{0} \cdot \nabla_{x} \bar{\eta}_{1}=G_{0}\left[\bar{\xi}_{1}\right] \\
& \bar{c}_{0} \cdot \nabla_{x} \bar{\xi}_{1}=-\bar{\eta}_{1} .
\end{aligned}
$$

In light of (Equation 5.0.1), we have

$$
\left(\begin{array}{cc}
\bar{c}_{0} \cdot \nabla_{x} & -G_{0} \\
1 & \bar{c}_{0} \cdot \nabla_{x}
\end{array}\right)\left(\begin{array}{l}
\bar{\eta}_{1} \\
\bar{\xi}_{1}
\end{array}\right)=\mathbf{0} .
$$

Since $\bar{\eta}_{1}$ and $\bar{\xi}_{1}$ are not time-dependent, we expand them in Fourier series of the form:

$$
\bar{\eta}_{1}(x)=\sum_{k \in \Gamma^{\prime}} d_{k} e^{i k \cdot x}, \quad \bar{\xi}_{1}(x)=\sum_{k \in \Gamma^{\prime}} a_{k} e^{i k \cdot x}
$$


We must therefore solve

$$
\left(\begin{array}{cc}
\bar{c}_{0} \cdot(i k) & -|k| \\
1 & \bar{c}_{0} \cdot(i k)
\end{array}\right)\left(\begin{array}{l}
d_{k} \\
a_{k}
\end{array}\right)=\mathbf{0}
$$

at every wavenumber $k \in \Gamma^{\prime}$. Non-trivial solutions exist only when a wavenumber $k_{1}$ and velocity $\bar{c}_{0}$ render the matrix singular:

$$
0=-\left(\bar{c}_{0} \cdot k_{1}\right)^{2}+\left|k_{1}\right|=-\left(\bar{c}_{0} \cdot k_{1}\right)^{2}+\omega_{k_{1}}^{2}
$$

This represents one equation for two unknown values of $\bar{c}_{0} \in \mathbf{R}^{2}$, so we may specify a second, linearly independent, wavenumber $k_{2} \in \Gamma^{\prime}$ and demand

$$
0=-\left(\bar{c}_{0} \cdot k_{2}\right)^{2}+\left|k_{2}\right|=-\left(\bar{c}_{0} \cdot k_{2}\right)^{2}+\omega_{k_{2}}^{2}
$$

Solving for $\omega_{k_{1}}$ and $\omega_{k_{2}}$, we have the linear system

$$
\left(\begin{array}{cc}
k_{1}^{(1)} & k_{1}^{(2)} \\
k_{2}^{(1)} & k_{2}^{(2)}
\end{array}\right)\left(\begin{array}{c}
\bar{c}_{0}^{(1)} \\
\bar{c}_{0}^{(2)}
\end{array}\right)=\left(\begin{array}{l}
\omega_{k_{1}} \\
\omega_{k_{2}}
\end{array}\right), \quad \overline{c_{0}}=\left(\begin{array}{c}
{\overline{c_{0}}}^{(1)} \\
{\overline{c_{0}}}^{(2)}
\end{array}\right), \quad k_{j}=\left(\begin{array}{c}
k_{j}^{(1)} \\
k_{j}^{(2)}
\end{array}\right)
$$

for $j=1,2$.

In this present study we select $k_{1}=(\sin (\theta), \cos (\theta))^{T}$ and $k_{2}=(\sin (\theta),-\cos (\theta))^{T}$ to yield

$$
\bar{c}_{0}=\left(\begin{array}{c}
1 / \sin (\theta) \\
0
\end{array}\right)
$$


With this choice of velocity $\bar{c}_{0}$, we find solutions to (Equation 5.0.3) at $k=k_{1}, k_{2}$ of the form

$$
\left(\begin{array}{c}
d_{k} \\
a_{k}
\end{array}\right)=A\left(\begin{array}{c}
|k| \\
i \bar{c}_{0} \cdot k
\end{array}\right)=A\left(\begin{array}{l}
1 \\
i
\end{array}\right)
$$

for any $A=(\rho / 2) e^{i \phi} \in \mathbf{C}$, where $\rho \in \mathbf{R}$, together with those of the form

$$
\left(\begin{array}{l}
d_{-k} \\
a_{-k}
\end{array}\right)=\left(\begin{array}{l}
\bar{d}_{k} \\
\bar{a}_{k}
\end{array}\right)=\bar{A}\left(\begin{array}{c}
1 \\
-i
\end{array}\right) .
$$

For our choices of wave number, we have

$$
\begin{aligned}
\bar{\eta}_{1}(x) & =d_{k_{1}} e^{i k_{1} \cdot x}+d_{k_{2}} e^{i k_{2} \cdot x}+d_{\bar{k}_{1}} e^{-i k_{1} \cdot x}+d_{\bar{k}_{2}} e^{-i k_{2} \cdot x} \\
& =\frac{\rho_{1}}{2} e^{i \phi_{1}} e^{i k_{1} \cdot x}+\frac{\rho_{2}}{2} e^{i \phi_{2}} e^{i k_{2} \cdot x}+\frac{\rho_{1}}{2} e^{-i \phi_{1}} e^{-i k_{1} \cdot x}+\frac{\rho_{2}}{2} e^{-i \phi_{2}} e^{-i k_{2} \cdot x} \\
& =\rho_{1} \cos \left(k_{1} \cdot x+\phi_{1}\right)+\rho_{2} \cos \left(k_{2} \cdot x+\phi_{2}\right)
\end{aligned}
$$

and

$$
\begin{aligned}
\bar{\xi}_{1}(x) & =a_{k_{1}} e^{i k_{1} \cdot x}+a_{k_{2}} e^{i k_{2} \cdot x}+a_{\bar{k}_{1}} e^{-i k_{1} \cdot x}+a_{\bar{k}_{2}} e^{-i k_{2} \cdot x} \\
& =\frac{i \rho_{1}}{2} e^{i \phi_{1}} e^{i k_{1} \cdot x}+\frac{i \rho_{2}}{2} e^{i \phi_{2}} e^{i k_{2} \cdot x}-\frac{i \rho_{1}}{2} e^{-i \phi_{1}} e^{-i k_{1} \cdot x}-\frac{i \rho_{2}}{2} e^{-i \phi_{2}} e^{-i k_{2} \cdot x} \\
& =-\rho_{1} \sin \left(k_{1} \cdot x+\phi_{1}\right)-\rho_{2} \sin \left(k_{2} \cdot x+\phi_{2}\right)
\end{aligned}
$$


We now have the quadruply parameterized family of solutions to (Equation 5.0.1)

$$
\begin{aligned}
& \bar{\eta}_{1}(x)=\rho_{1} \cos \left(k_{1} \cdot x+\phi_{1}\right)+\rho_{2} \cos \left(k_{2} \cdot x+\phi_{2}\right) \\
& \bar{\xi}_{1}(x)=-\rho_{1} \sin \left(k_{1} \cdot x+\phi_{1}\right)-\rho_{2} \sin \left(k_{2} \cdot x+\phi_{2}\right)
\end{aligned}
$$

for any choices of $\rho_{j}, \phi_{j}$.

We lose no generality by setting $\phi_{j}=0$ as this simply sets the maximum for $\bar{\eta}_{1}$ at the origin, and if we express the amplitudes $\left(\rho_{1}, \rho_{2}\right)$ in polar coordinates $(\tau, \sigma)$ via

$$
\rho_{1}=\tau \cos (\sigma), \quad \rho_{2}=\tau \sin (\sigma),
$$

then we may set $\tau=1$ by varying $\alpha$. However, we are free to vary the "skewness ratio" $\sigma$ away from $\pi / 4$, which is the linearization of the classical SCWs, to any value $0<\sigma<\pi / 2$, which is the linear part of a GSCW. (Note that $\sigma=0, \pm \pi / 2$ constitute Stokes waves in rotated coordinates.)

The procedure we employ for computing the base traveling waves is due to Nicholls and Reitich (13) and is perturbative in nature. Refer to Chapter 6 for more details. In short this approach is based upon the strongly convergent perturbation expansions (40)

$$
\bar{c}=\bar{c}(\varepsilon)=\bar{c}_{0}+\sum_{n=1}^{\infty} \bar{c}_{n} \varepsilon^{n}, \quad \bar{\eta}=\bar{\eta}(x ; \varepsilon)=\bar{\eta}_{1}(x) \varepsilon+\sum_{n=2}^{\infty} \bar{\eta}_{n} \varepsilon^{n}, \quad \bar{\xi}=\bar{\xi}(x ; \varepsilon)=\bar{\xi}_{1}(x) \varepsilon+\sum_{n=2}^{\infty} \bar{\xi}_{n} \varepsilon^{n} .
$$


The corrections $\left\{\bar{c}_{n}\right\}, n \geq 1$, and $\left\{\bar{\eta}_{n}, \bar{\xi}_{n}\right\}, n \geq 2$, provide the "nonlinearization" of the traveling profiles, and help further distinguish our GSCWs from the classical SCWs considered by other authors. Surface and contour plots of GSCWs are available in Chapter 7. 


\section{CHAPTER 6}

\section{NUMERICAL METHOD AND PARALLEL APPROACH}

\subsection{Numerical Method}

The goal is to complete a numerical simulation of the spectral data $\{\lambda, \zeta(x), \psi(x)\}$ from the stability eigenproblem (Equation 2.7.1). The first task is to produce high-order approximations of traveling wave solutions $\{\bar{c}, \bar{\eta}, \bar{\xi}\}$ to feed into our spectral stability code, of which there are a variety of methods (41). With our reduction in dimension, we could choose among the boundary integral/element methods (BIM/BEM) (42) or high-order (HOS) spectral methods (43). The common theme among both is the premise of unknown surface quantities. It is the reduction in dimension to the surface that makes them preferable to volumetric methods.

The work on HOS methods that is closely related to our HOS method of choice is that of Roberts (8), Roberts and Peregrine (9), and Marchant and Roberts (22). These Field Expansions (FE) methods, which work under the premise that the potential, surface, and speed can be expanded in convergent Taylor series in $\epsilon$, suffer from severe limitations. For one, their computational complexity does not scale well in relation to their discretization parameters. For two-dimensional surfaces, if $N$ orders are approximated with $N_{x_{1}} \times N_{x_{2}}$ discretization points, the execution time is $\mathcal{O}\left(N^{3} N_{x_{1}}^{2} N_{x_{2}}^{2}\right)$ (14). Secondly, these algorithms, like many boundary perturbation methods, experience instability at high orders. This ill-conditioning is due to the need to estimate the spatial derivatives of the velocity potential at increasingly higher orders, 
based upon the explicit recursions (14) that define the coefficients in the FE method, and applying the triangle inequality (in an appropriate function space). However, these bounds can not yield a finite rate of growth under any norm for the solutions precisely because they destroy cancellations which which underly the FE recursions (13).

To resolve these issues, we use the stable HOS boundary perturbation method of Nicholls and Reitich (13). It uses the method of Transformed Field Expansions. A very brief summary of the rationale behind this method is now given.

For infinite depth, this method begins with the traveling wave equations for waves translating uniformly with speed $c \in \mathbf{R}^{2}$. It then uses a transparent boundary condition and augmented velocity potential to derive the equivalent form in a truncated problem domain:

$$
\begin{array}{ll}
\Delta \varphi=0 & -a<y<\eta(x) \\
\partial_{y} \varphi(x,-a)-|D| \varphi(x,-a)=0 & \\
c \cdot \nabla_{x} \eta+\nabla_{x} \eta \cdot \nabla_{x} \varphi-\partial_{y} \varphi=0 & \text { at } y=\eta \\
c \cdot \nabla_{x} \varphi+\frac{1}{2} \nabla \varphi \cdot \nabla \varphi+g \eta=0 & \text { at } y=\eta,
\end{array}
$$

where $\|\eta\|_{L^{\infty}}<a<\infty$. This restatement yields a uniform statement of the water wave problem for any depth. 
The next step is to transform the problem domain by flattening the surface $\eta$ with the change of variables (Equation 6.1.2)

$$
x^{\prime}=x, \quad y^{\prime}=a\left(\frac{y-\eta}{y+\eta}\right)
$$

set $u\left(x^{\prime}, y^{\prime}\right):=\varphi\left(x^{\prime}, \frac{(a+\eta) y^{\prime}}{a+\eta}\right)$, and upon completion drop the primes to get equations of the form

$$
\begin{array}{ll}
\Delta u=F(x, y) & -a<y<0 \\
\partial_{y} u(x,-a)-|D| u(x,-a)=J(x) & \\
c_{0} \cdot \nabla_{x} \eta-\partial_{y} u=Q(x) & \text { at } y=0 \\
c_{0} \cdot \nabla_{x} u+g \eta=R(x) & \text { at } y=0,
\end{array}
$$

where $c_{0}$ is carefully chosen by analyzing a linearization of (Equation 6.1.2) about the trivial state $\varphi=\eta=0$ for any velocity $c$ and searching for solution branches via bifurcation theory. The definitions of $F(x, y), J(x), Q(x)$, and $R(x)$ can be found in (13).

In order to solve (Equation 6.1.2), the following are posited for $\epsilon>0$ and inserted into (Equation 6.1.2)

$$
\begin{gathered}
c(\varepsilon)=\sum_{n=0}^{\infty} c_{n} \varepsilon^{n}, \quad \eta(x ; \varepsilon)=\sum_{n=1}^{\infty} \eta_{n}(x) \varepsilon^{n}, \\
u(x, y ; \varepsilon)=\sum_{n=1}^{\infty} u_{n}(x, y) \varepsilon^{n}
\end{gathered}
$$


resulting in a problem for each $n>0$ :

$$
\begin{array}{ll}
\Delta u_{n}=F_{n}(x, y) & -a<y<0 \\
\partial_{y} u_{n}(x,-a)-|D| u_{n}(x,-a)=J_{n}(x) & \\
c_{0} \cdot \nabla_{x} \eta_{n}-\partial_{y} u_{n}=Q_{n}(x)-c_{n-1} \cdot \nabla_{x} \eta_{1} & \text { at } y=0 \\
c_{0} \cdot \nabla_{x} u_{n}+g \eta_{n}=R_{n}(x)-c_{n-1} \cdot \nabla_{x} u_{1} & \text { at } y=0,
\end{array}
$$

where the $c_{n-1}$ are chosen for solvability. The definitions of $F_{n}(x, y), J_{n}(x), Q_{n}(x)$, and $R_{n}(x)$ can be found in (13).

The equations in (Equation 6.1.3) almost completely prescribes the TFE algorithm. In order to code this, an approximation to the full solution is necessary. This is done by inserting

$$
\begin{gathered}
c^{N}(\varepsilon)=\sum_{n=0}^{N} c_{n} \varepsilon^{n}, \quad \eta^{N, N_{x}}(x ; \varepsilon)=\sum_{n=0}^{N} \sum_{|p|<\frac{N_{x}}{2}} \eta_{n, p} e^{i p \cdot x} \varepsilon^{n} \\
u^{N, N_{x}, N_{y}}(x, y ; \varepsilon)=\sum_{n=0}^{N} \sum_{|p|<\frac{N_{x}}{2}} \sum_{l=0}^{N_{y}} u_{n, p, l} e^{i p \cdot x} T_{l}(y) \varepsilon^{n}
\end{gathered}
$$

into (Equation 6.1.3) and using the Fourier collocation-Chebyshev-Tau method (44) to yield a linear system of equations at each order $n$ and wavenumber $p$, which the $u_{n, p, l}$ and $\eta_{n, p}$ must satisfy, to produce the coefficients $\left\{c_{n}, \eta_{p, n}, u_{p, l, n}\right\} . T_{l}$ is the $l$-th Chebyshev polynomial, $p \in$ $P\left(\Gamma^{\prime}\right)$, and $\left\{N, N_{x}, N_{y}\right\}$ are the perturbation, horizontal, and vertical discretization parameters, respectively. 
For our purposes, an approximation to the surface velocity potential is more relevant and can be recovered from

$$
\xi^{N, N_{x}}(x ; \varepsilon)=u^{N, N_{x}, N_{y}}(x, 0 ; \varepsilon),
$$

since the transformation maps $y=\eta$ to $y^{\prime}=0$.

The execution time of the method of Nicholls and Reitich, for a grid with $N_{x_{1}} \times N_{x_{2}}$ discretization points is only $\mathcal{O}\left(N N_{x_{1}} N_{x_{2}} \log \left(N_{y}\right) N_{y}+N^{2} N_{x_{1}} N_{x_{2}}, N_{y}\right)$. This algorithm is stable and eliminates the effects of ill-conditioning experienced by the FE approach. It is therefore a more reliable estimator of quantities relevant to traveling waves such as shape, energy, and frequency.

The second task is to approximate solutions of the spectral stability problem (Equation 2.7.1). For this we simply use a Fourier collocation approach and simulate $\zeta(x)$ and $\psi(x)$ with

$$
\zeta^{N_{x}}=\sum_{|k|<\frac{N_{x}}{2}} \tilde{\zeta}_{k} e^{i(k+p) \cdot x}, \quad \psi^{N_{x}}=\sum_{|k|<\frac{N_{x}}{2}} \tilde{\psi}_{k} e^{i(k+p) \cdot x},
$$

respectively. These expansions are inserted into (Equation 2.7.1) and enforced at the equally spaced grid points $P(\Gamma)$. This is also straightforward for $A(\bar{\eta}), B(\bar{\eta}, \bar{\xi}), A_{\eta}(\bar{\eta}), B_{\eta}(\bar{\eta}, \bar{\xi})$, and $B_{\xi}(\bar{\eta}, \bar{\xi})$. The derivatives are simulated spectrally and the nonlinearities are evaluated with fast convolutions.

For the computation of $G(\bar{\eta})$ and $G_{\eta}(\bar{\eta})$, we use the algorithms developed by Nicholls and Fazioli $(33 ; 34)$. The essence of these algorithms is to evaluate the terms $G_{n}$ and $G_{n}^{(1)}$ from 
Chapter 3 via spectral Fourier multipliers and fast convolutions, followed by a summation of truncated Taylor series. For our simulations, we truncated the series for these at $N=8$.

\subsection{Parallel Approach}

Embarrassingly parallel (45) $\mathrm{C}++$ code was developed to read in the traveling wave information as described in Chapter 6 and then compute the matrix representation of the linearized water wave operator $\mathcal{A}(x)$ about the traveling wave solutions for each quasi period $p$. Once the matrix representation of the linear operator $\mathcal{A}$ has been formed for each $p$, we use the LAPACK routine zgeevx to find the spectrum for each choice of $\alpha$. For each value of $\alpha$, we compute the real part of the eigenvalue with the largest real part

$$
r_{\max }(\alpha):=\max _{p \in P\left(\Gamma^{\prime}\right)}\{\operatorname{Re}\{\lambda\} \mid \lambda \in \operatorname{spec}(\mathcal{A})\}
$$

These values are plotted in Chapter 7 for a sampling of $\alpha$ and a particular subset of the $p \in P\left(\Gamma^{\prime}\right)$ determined by the computational resources available, an iMac running Snow Leopard with 2 quad-core $2.26 \mathrm{GHz}$ Intel Xeon processors and $16 \mathrm{~GB}$ of memory. The jobs were run using OpenMPI version 1.2.8.

More precisely, within each configuration $\left(\rho_{1}, \rho_{2}, \theta\right)$, for each $\alpha$, one parallel job was executed. Once the quasi periods and traveling wave information were read in, within this job, an embarrassingly parallel approach via MPI was used to divide up each execution among the $p=\left(p_{1}, p_{2}\right)$. The traveling wave and quasi period information were scattered and broadcast to the child processes. Since we used the same $10 \times 10$ grid of quasi periods for all simulations, 
20 values of $p$ were handled in each of 5 child processes. The rationale behind this approach was simply to speed up processing time to allow as much data as possible to be collected and analyzed.

What makes the values of $p$ a natural choice for dividing up the processing is the computations involving one particular pair $\left(p_{1}, p_{2}\right)$ do not affect the computations involving other choices for $p$. Thus there is no need for interprocess communication or the need for one child process to wait for another child to reach a particular state to perform its work. At the end, we simply collated all of the output data, which consists of the eigenvalues for each $p$ and a file containing the maximum real part of the eigenvalues obtained for each $p$. For example, the parallel version for $\frac{\rho_{1}}{\rho_{2}}=\frac{1}{4}$ and $\theta=60^{\circ}$ for $\alpha=.02$ completed execution across all five child processes in $7 \mathrm{~h} 14 \mathrm{~m} 3.16 \mathrm{~s}$, while the serial version for the same configuration took $20 \mathrm{~h} 02 \mathrm{~m} 18.9 \mathrm{~s}$.

For all of our simulations, we used the following common values: 


\begin{tabular}{|c|c|c|}
\hline Variable & Value & Meaning \\
\hline \hline$N_{x_{1}}$ & 32 & Discretization points in the $x_{1}$ direction. \\
\hline$N_{x_{2}}$ & 32 & Discretization points in the $x_{2}$ direction. \\
\hline$N_{y}$ & 24 & Discretization points in the $y$ direction \\
\hline$N$ & 10 & Perturbation order. \\
\hline$h$ & 10000 & Simulation of infinite depth. \\
\hline$N D N O$ & 8 & Order for truncated series for $G$ and its first variation. \\
\hline$N u m p$ & 100 & Number of quasi periods $p$. \\
\hline$p=\left(p_{1}, p_{2}\right)$ & $0 \leq p_{i} \leq 0.5$ & Ranges for quasiperiods. \\
\hline
\end{tabular}

TABLE I

COMMON VALUES ACROSS ALL SIMULATIONS 


\section{CHAPTER 7}

\section{NUMERICAL RESULTS}

\subsection{GSCW Surface and Contour Plots}

Figures 1 through 6 display surface and contour plots of traveling GSCW forms for the

underlying geometry $\theta=45^{\circ}$ of the square period cell, with $k_{1}=\left(\frac{1}{\sqrt{2}}, \frac{1}{\sqrt{2}}\right)$ and $k_{2}=\left(\frac{1}{\sqrt{2}},-\frac{1}{\sqrt{2}}\right)$, and "skewness" values $\frac{\rho_{1}}{\rho_{2}}=\cot (\sigma)=1, \frac{1}{2}$, and $\frac{1}{4}$. It is clear how asymmetric these wave forms can become, even with $\theta=45^{\circ}$. Figures 1 and 2 depict a classical SCW, while the Figures 3 through 6 depict GSCWs that tend toward the physical form of the Stokes wave $\cos \left(k_{2} \cdot x\right)$, shown in Figures 7 and 8. 


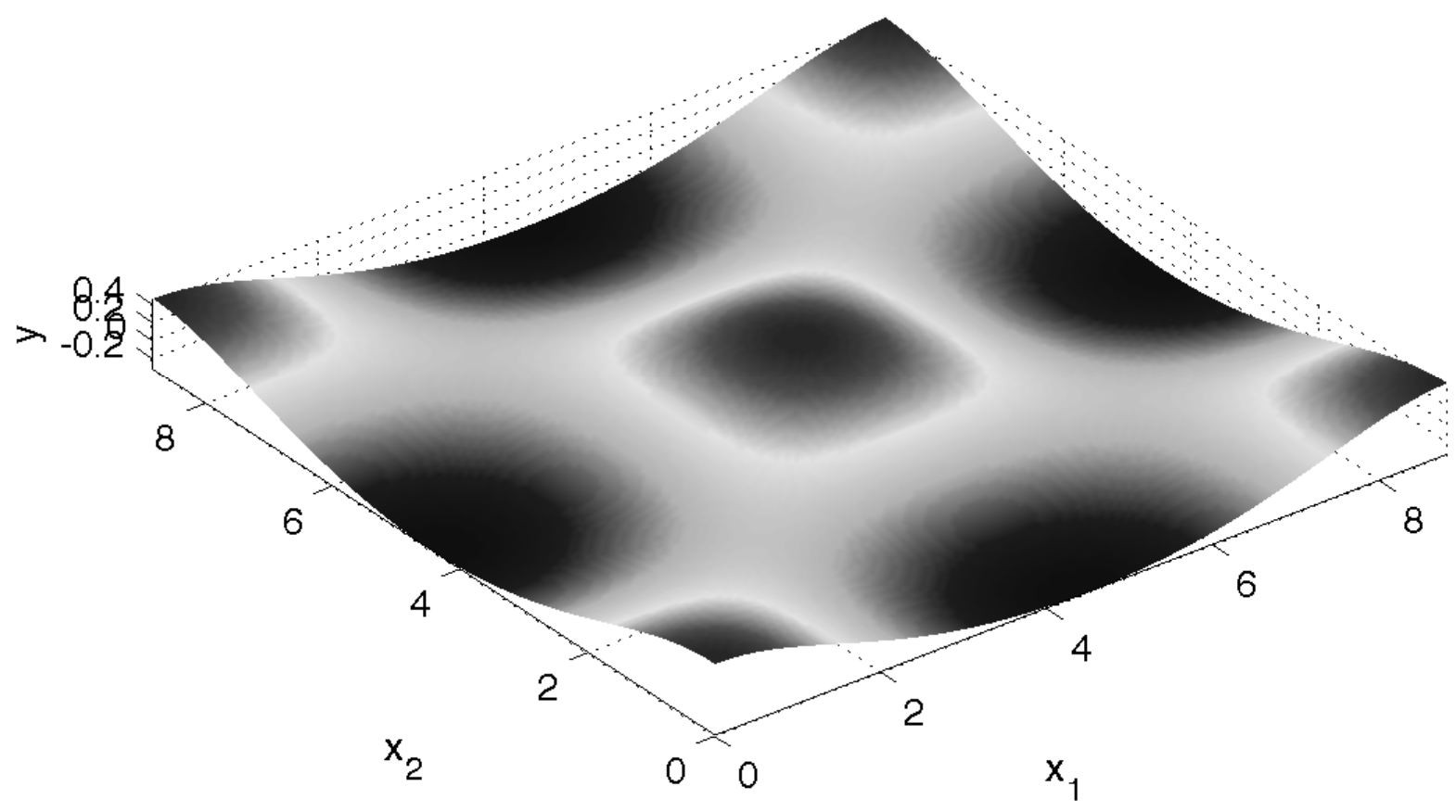

Figure 1. Surface plot of a Generalized Short-Crested Wave for $\frac{\rho_{1}}{\rho_{2}}=1$. 


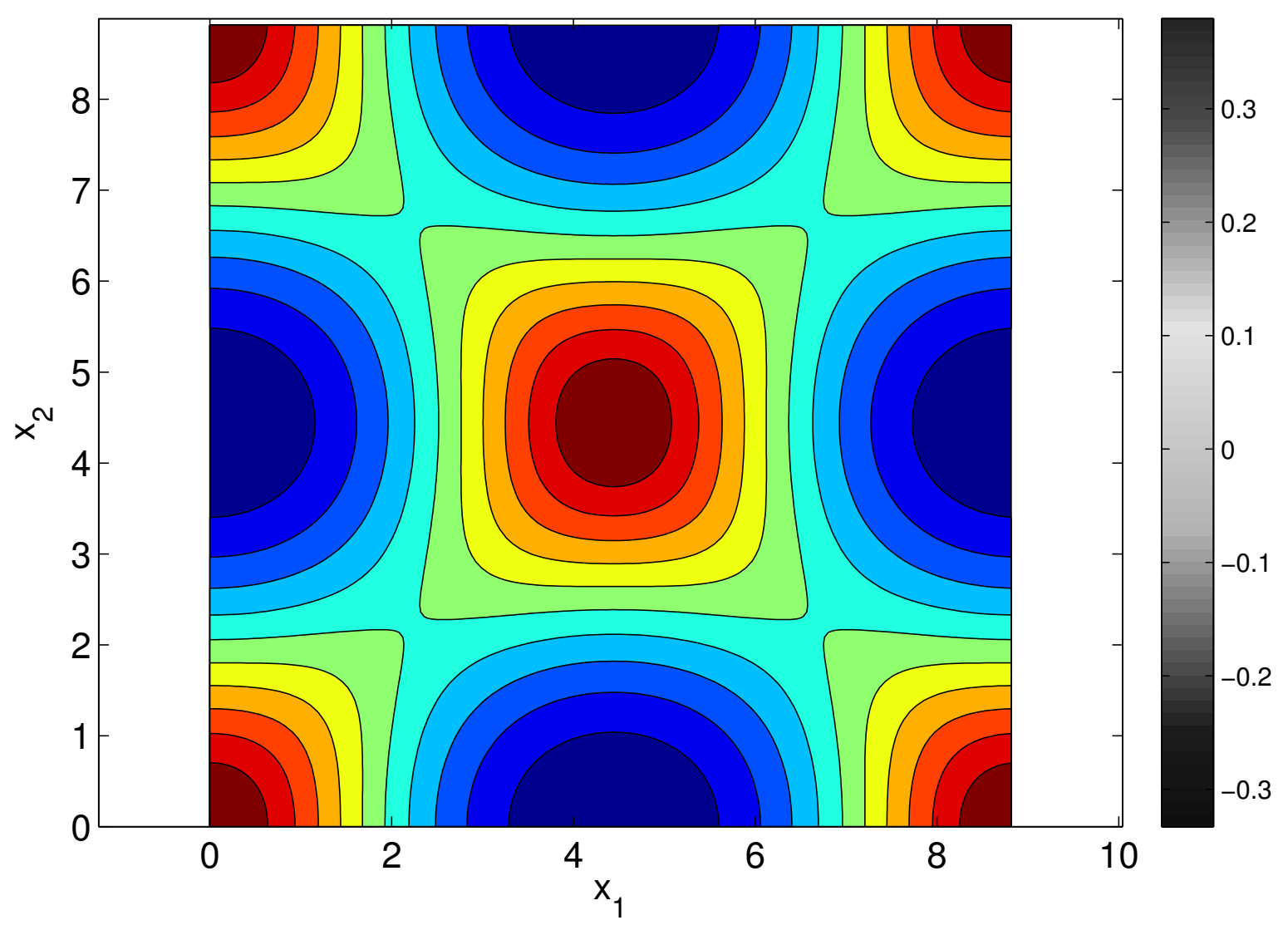

Figure 2. Contour plot of a Generalized Short-Crested Wave for $\frac{\rho_{1}}{\rho_{2}}=1$. 


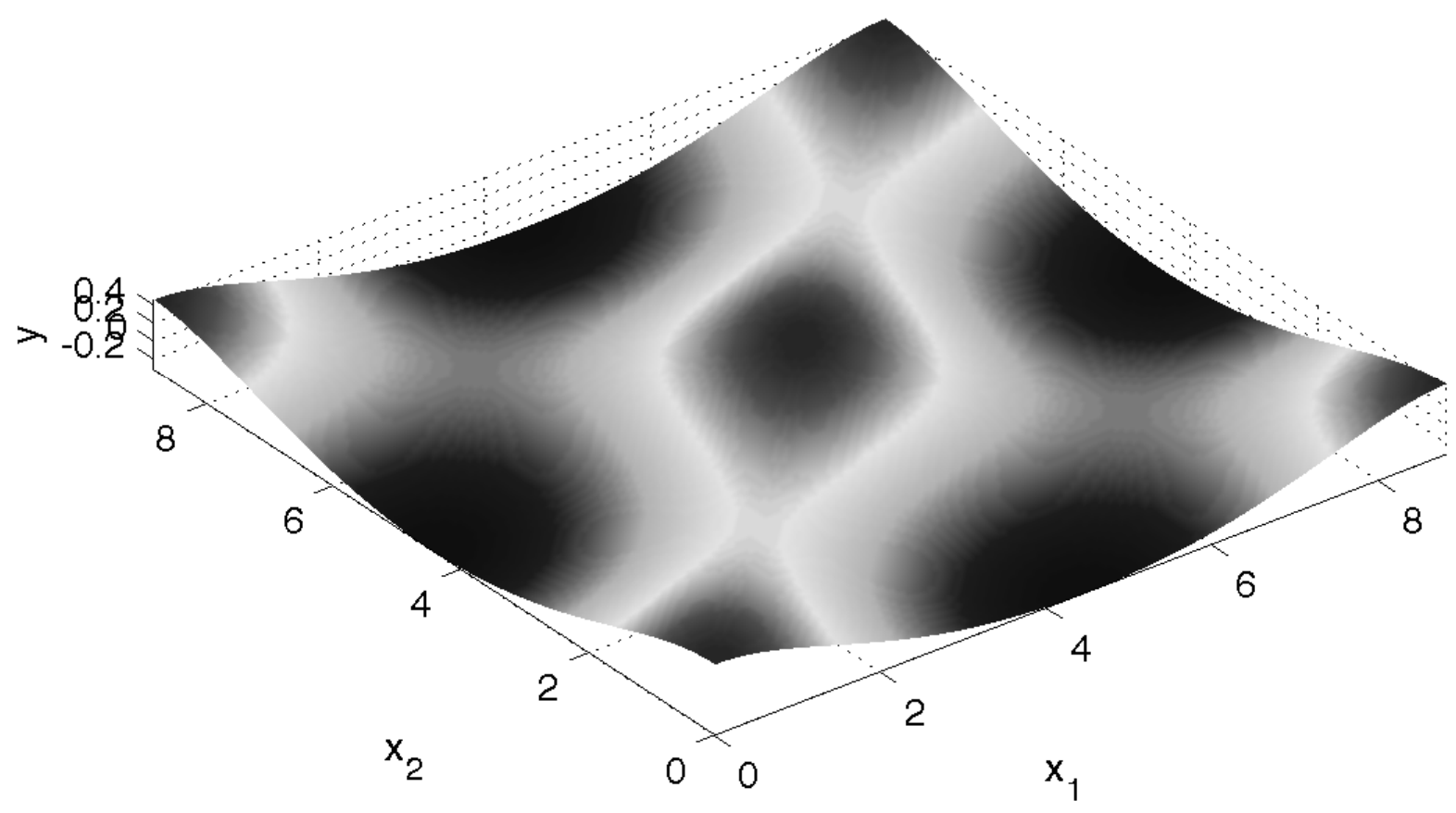

Figure 3. Surface plot of a Generalized Short-Crested Wave for $\frac{\rho_{1}}{\rho_{2}}=\frac{1}{2}$. 


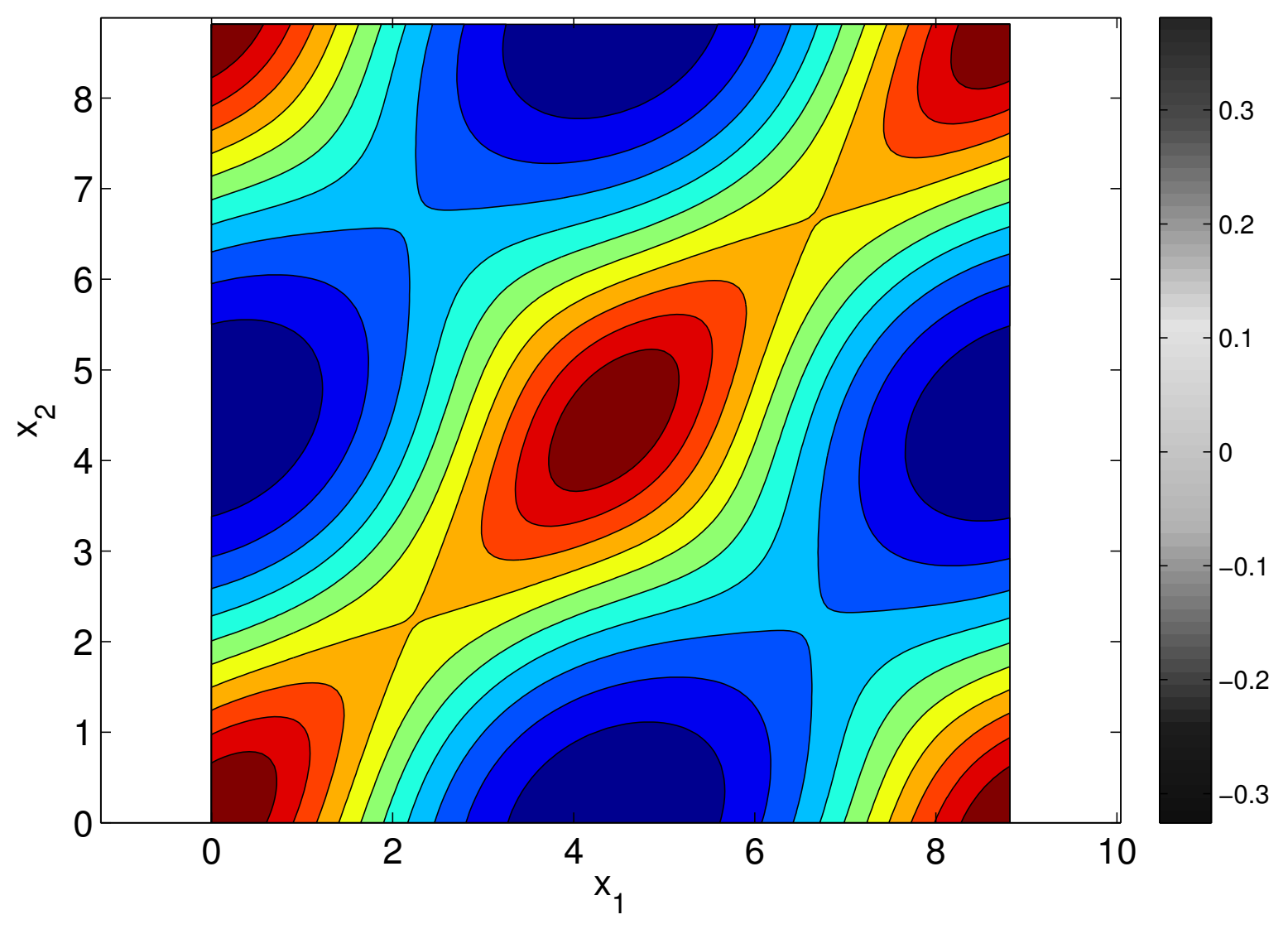

Figure 4. Contour plot of a Generalized Short-Crested Wave for $\frac{\rho_{1}}{\rho_{2}}=\frac{1}{2}$. 


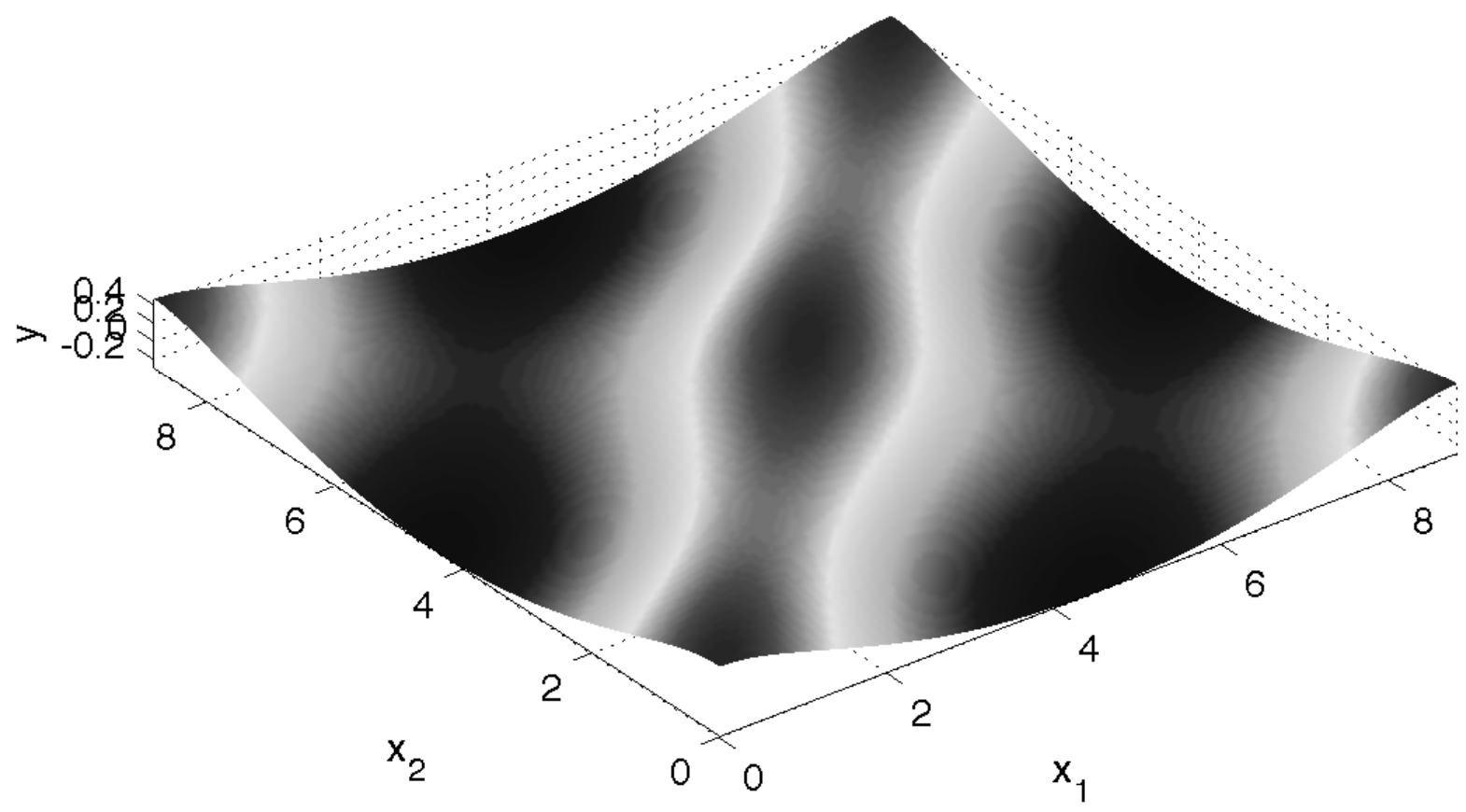

Figure 5. Surface plot of a Generalized Short-Crested Wave for $\frac{\rho_{1}}{\rho_{2}}=\frac{1}{4}$. 


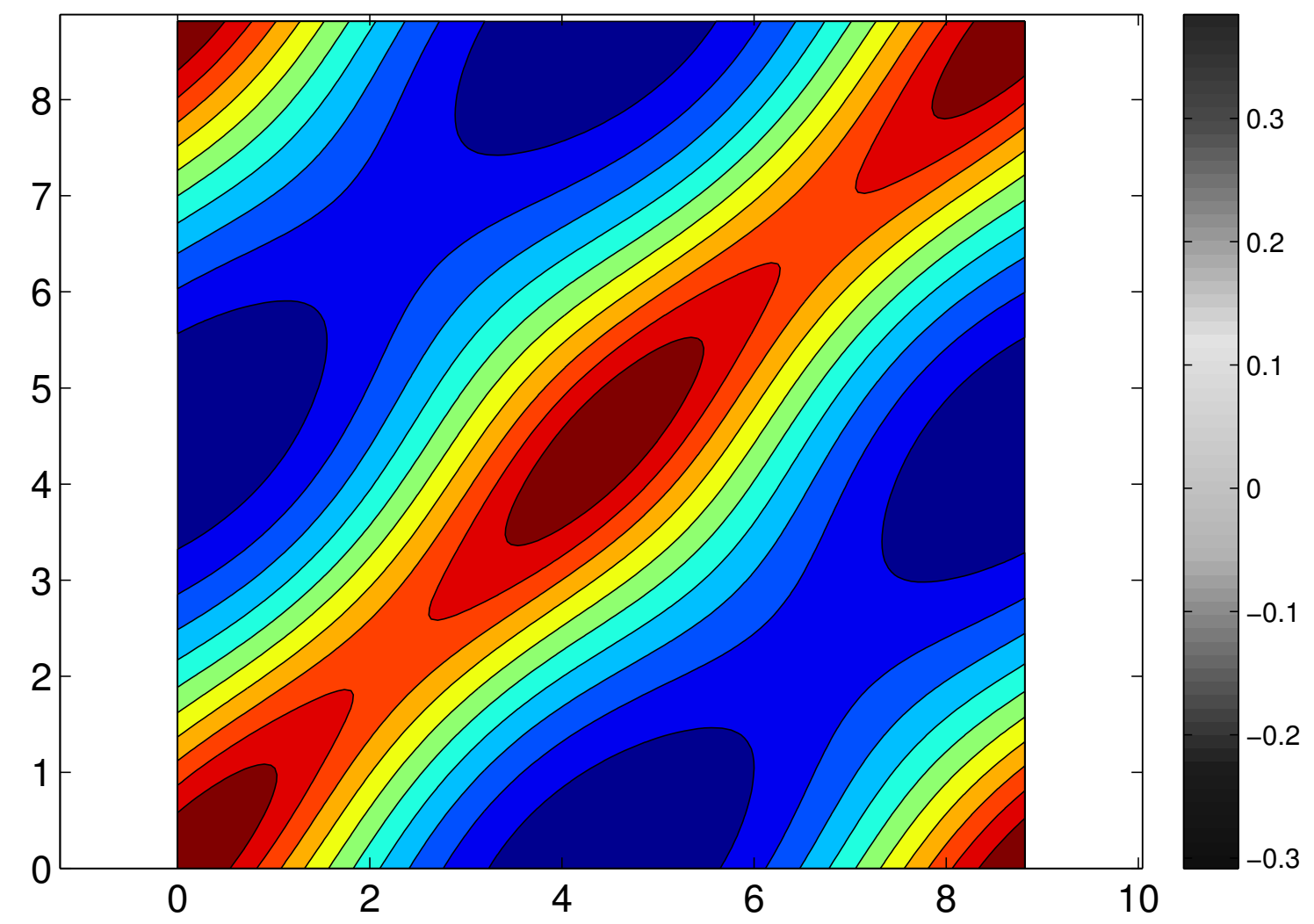

Figure 6. Contour plot of a Generalized Short-Crested Wave for $\frac{\rho_{1}}{\rho_{2}}=\frac{1}{4}$. 


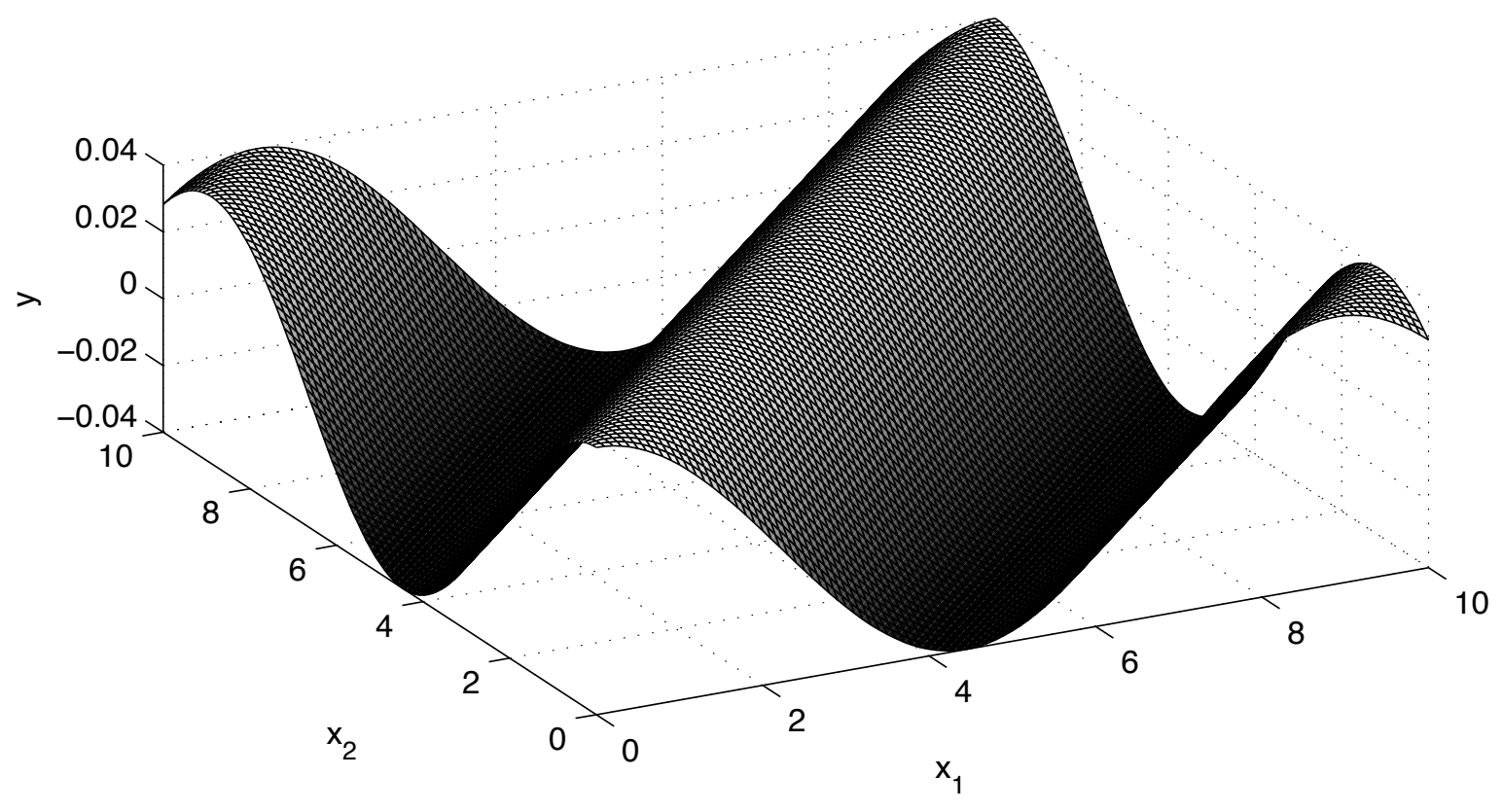

Figure 7. Stokes wave surface, .04 $\cos \left(k_{2} \cdot x\right)$. 


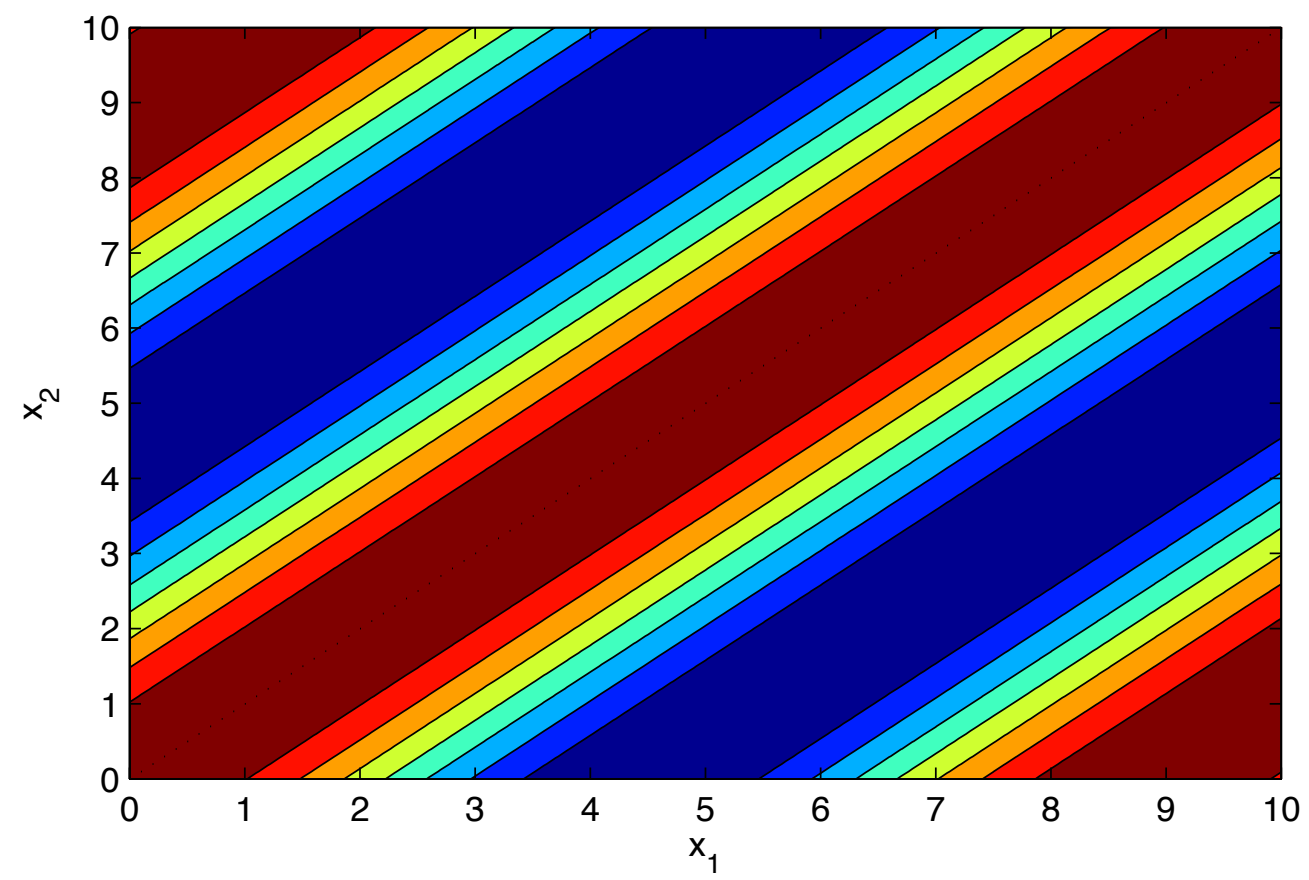

Figure 8. Stokes wave contours, $.04 \cos \left(k_{2} \cdot x\right)$.

\subsection{Numerical Simulations: Overview}

Numerical simulations were completed for three geometric configurations:

1. Square period cell: $\theta=45^{\circ}$.

2. Moderately skewed rectangular period cell: $\theta=60^{\circ}$.

3. Extremely skewed rectangular period cell: $\theta=75^{\circ}$.

Within each of the geometric configurations, three classes of GSCWs were simulated: 
1. SCW: $\frac{\rho_{1}}{\rho_{2}}=1$.

2. Moderately skewed GSCW: $\frac{\rho_{1}}{\rho_{2}}=\frac{1}{2}$.

3. Extremely skewed GSCW: $\frac{\rho_{1}}{\rho_{2}}=\frac{1}{4}$.

For each of the nine possible combinations of the above, the base traveling waves were computed for a sampling of $\alpha$ using the method of Nicholls and Reitich described in Chapter 6. For each $\alpha$, the quantity $r_{\max }\left(\theta, \rho_{1}, \rho_{2} ; \alpha\right)$ was determined for a $10 \times 10$ sampling of equallyspaced quasi-periods $p=\left(p_{1}, p_{2}\right), 0 \leq p_{i} \leq 0.5$, on the conjugate period cell $P\left(\Gamma^{\prime}\right)$.

The $r_{\max }\left(\theta, \rho_{1}, \rho_{2} ; \alpha\right)$ quantities for a given configuration represent the strength of the instabilities. To leading order, the surface perturbation will grow like

$$
C e^{r_{\max } t} \zeta(x)
$$

plus more slowly growing contributions. We now provide some detailed results for each geometric configuration.

\section{3 $\quad \underline{\theta}=45^{\circ}:$ Symmetric}

In Figures 9 through 11, we display $r_{\max }\left(45^{\circ}, \rho_{1}, \rho_{2} ; \alpha\right)$ versus $\alpha$ for $\frac{\rho_{1}}{\rho_{2}}=1, \frac{1}{2}$, and $\frac{1}{4}$. What's striking is for the range of quasiperiods $p$ chosen, there is a Zone of Instability for each of our choices of $\frac{\rho_{1}}{\rho_{2}}$, for which waves of smaller and larger amplitude are stable. $\left(10^{-7}\right.$ was chosen to be equivalent to 0 for all of our simulations.) As the ratio $\frac{\rho_{1}}{\rho_{2}}$ is decreased from 1 , the traveling waves become more unstable in that the instability arises for smaller values of $\alpha$ (less nonlinear waves). See Table II below. 


\begin{tabular}{|c|c|}
\hline$\frac{\rho_{1}}{\rho_{2}}$ & Interval \\
\hline \hline 1 & $0.01<\alpha<0.028$ \\
\hline$\frac{1}{2}$ & $0.008<\alpha<0.02$ \\
\hline$\frac{1}{4}$ & $0.006<\alpha<0.016$ \\
\hline
\end{tabular}

TABLE II

ZONES OF INSTABILITY FOR $\theta=45^{\circ}$ 


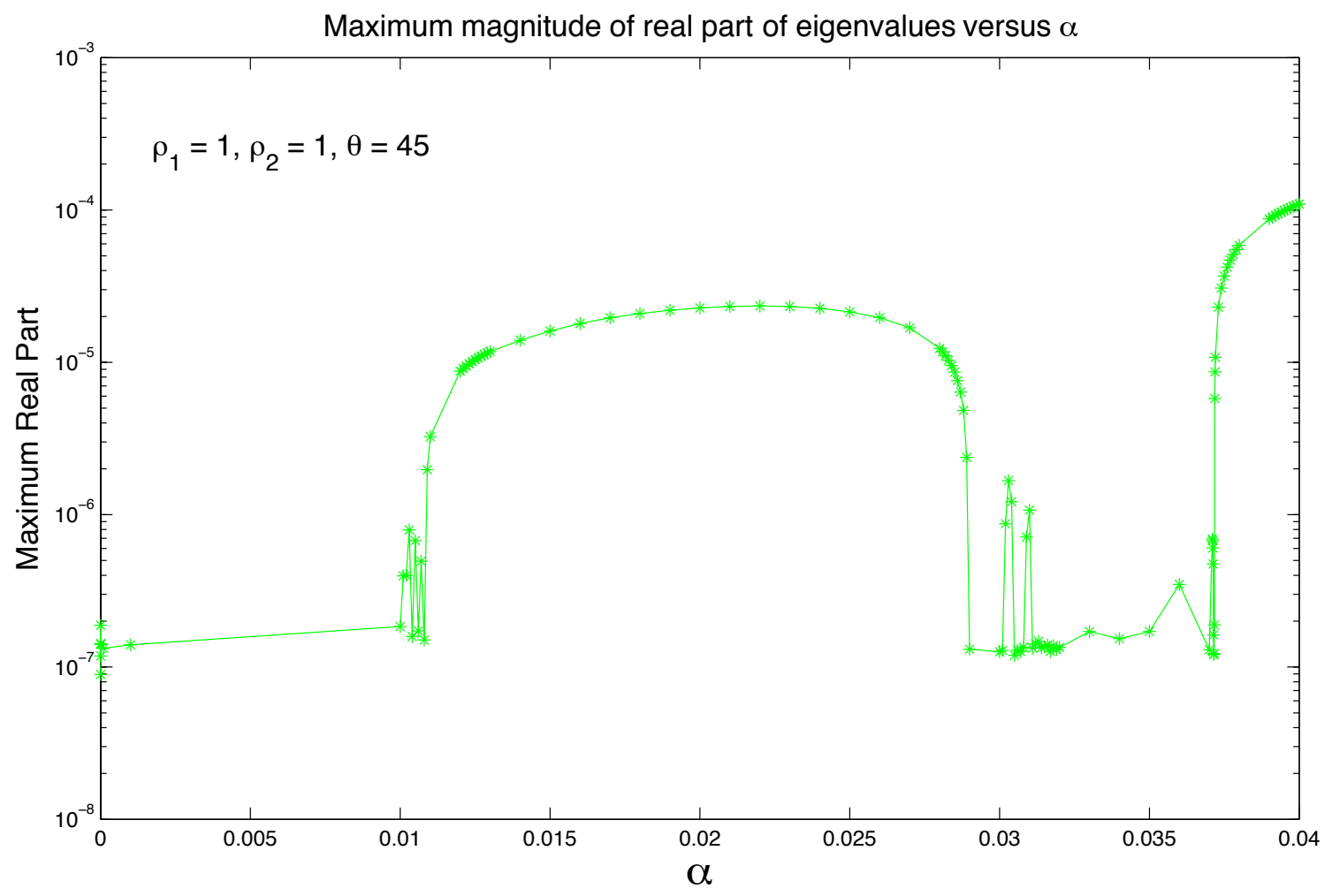

Figure 9. $r_{\max }\left(45^{\circ}, \rho_{1}, \rho_{2} ; \alpha\right)$ versus wave height/slope $\alpha$ for $\frac{\rho_{1}}{\rho_{2}}=1$. 


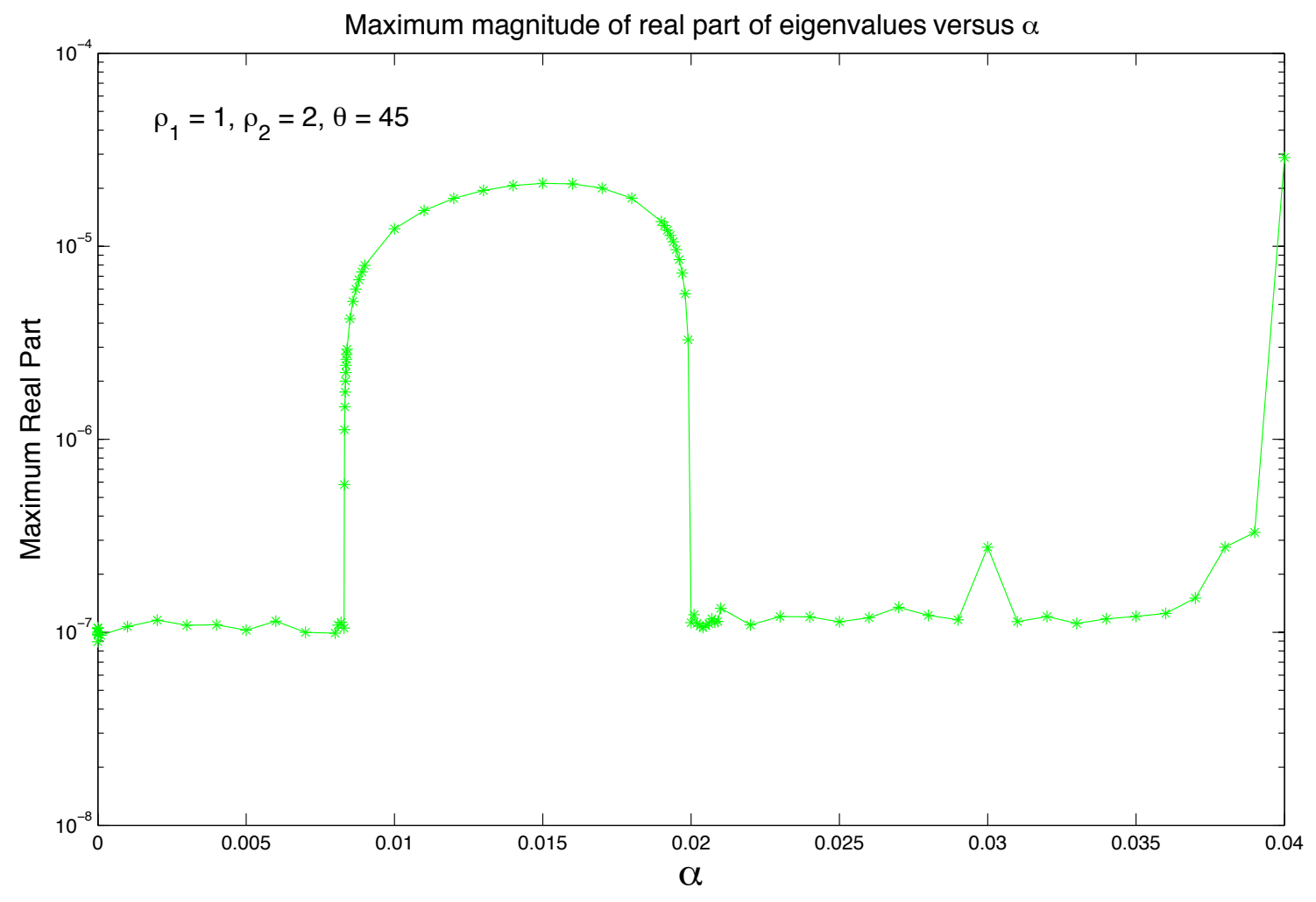

Figure 10. $r_{\max }\left(45^{\circ}, \rho_{1}, \rho_{2} ; \alpha\right)$ versus wave height/slope $\alpha$ for $\frac{\rho_{1}}{\rho_{2}}=\frac{1}{2}$. 


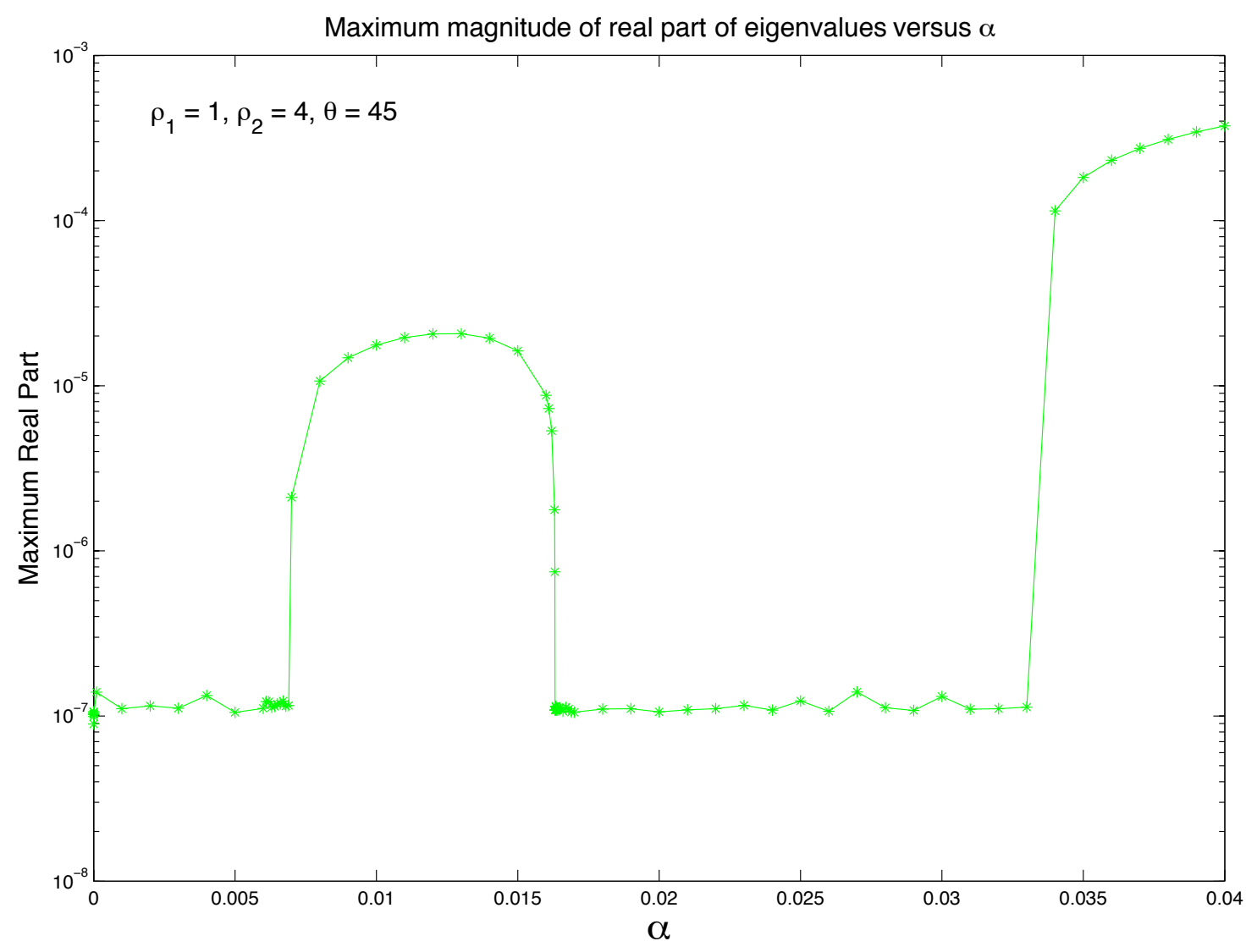

Figure 11. $r_{\max }\left(45^{\circ}, \rho_{1}, \rho_{2} ; \alpha\right)$ versus wave height/slope $\alpha$ for $\frac{\rho_{1}}{\rho_{2}}=\frac{1}{4}$. 
In Figures 12 through 14, we display plots of $r_{\max }\left(45^{\circ}, \rho_{1}, \rho_{2}, \alpha\right)$ versus $\left(p_{1}, p_{2}\right)$ to illustrate where the dominant instability occurs with respect to the quasiperiod for some chosen values of $\alpha$. See Table III below.

\begin{tabular}{|c|c|c|c|c|}
\hline$\frac{\rho_{1}}{\rho_{2}}$ & $\alpha$ & $p_{1}$ & $p_{2}$ & Max Level \\
\hline \hline 1 & 0.015 & 0.111111 & 0.222222 & $1.602882619805372 \times 10^{-5}$ \\
\hline$\frac{1}{2}$ & 0.015 & 0.111111 & 0.222222 & $2.120396833598851 \times 10^{-5}$ \\
\hline$\frac{1}{4}$ & 0.01 & 0.111111 & 0.222222 & $1.763374966930082 \times 10^{-5}$ \\
\hline
\end{tabular}

TABLE III

DOMINANT INSTABILITY FOR SOME CHOSEN VALUES OF $\alpha$ FOR $\theta=45^{\circ}$. 


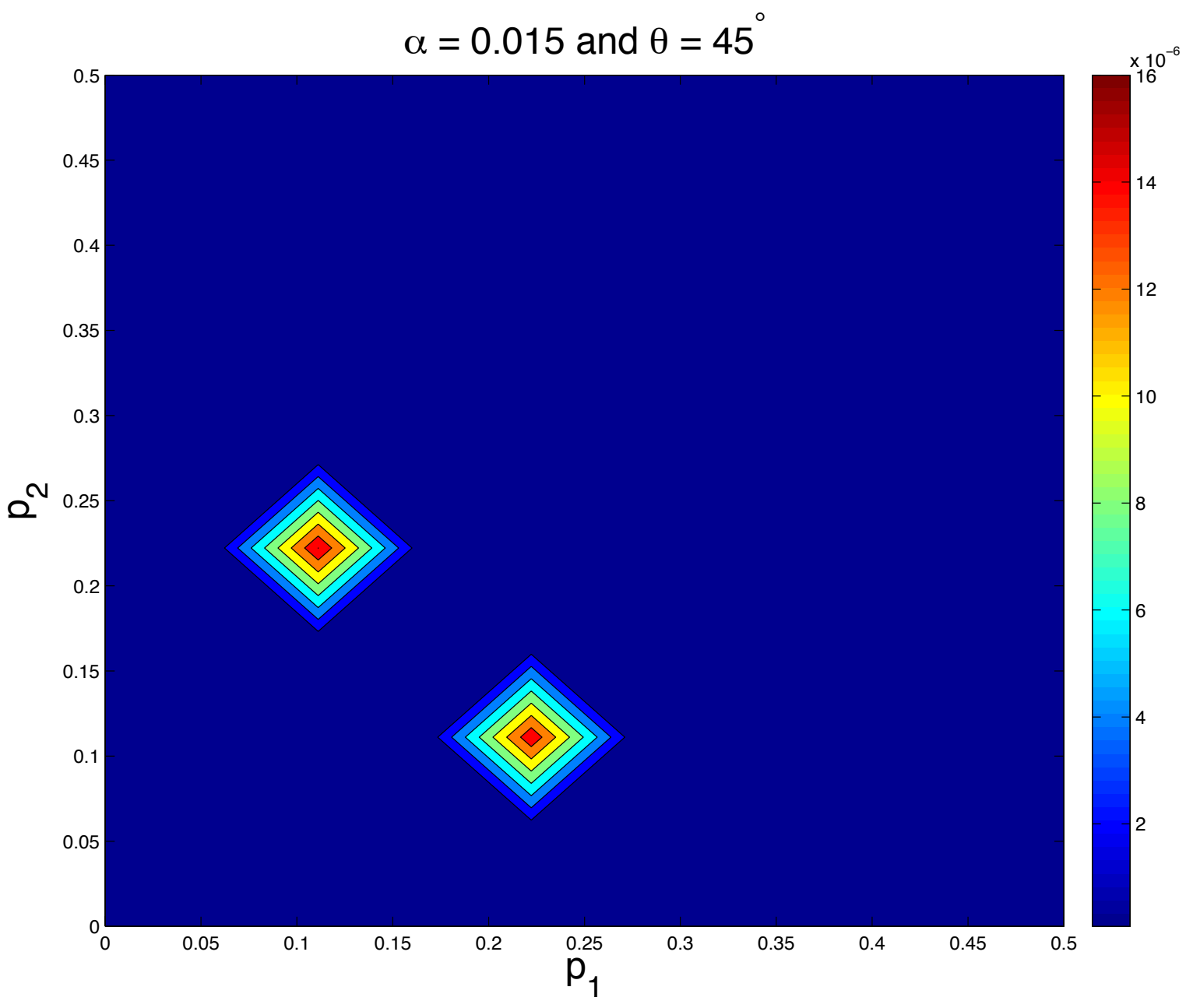

Figure 12. $r_{\max }\left(45^{\circ}, \rho_{1}, \rho_{2} ; 0.015\right)$ versus quasi period $\left(p_{1}, p_{2}\right)$ for $\frac{\rho_{1}}{\rho_{2}}=1$. 


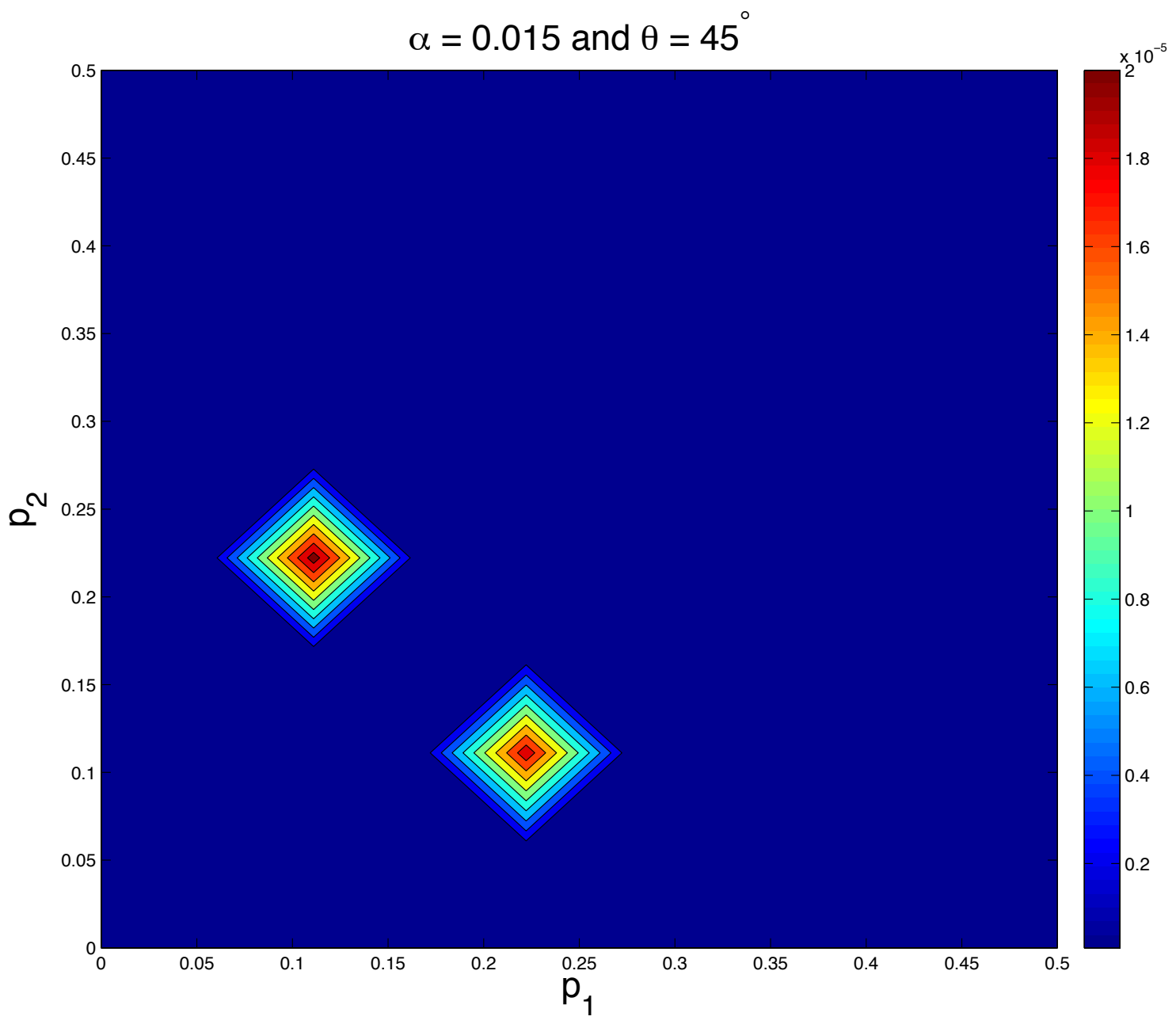

Figure 13. $r_{\max }\left(45^{\circ}, \rho_{1}, \rho_{2} ; 0.015\right)$ versus quasi period $\left(p_{1}, p_{2}\right)$ for $\frac{\rho_{1}}{\rho_{2}}=\frac{1}{2}$. 


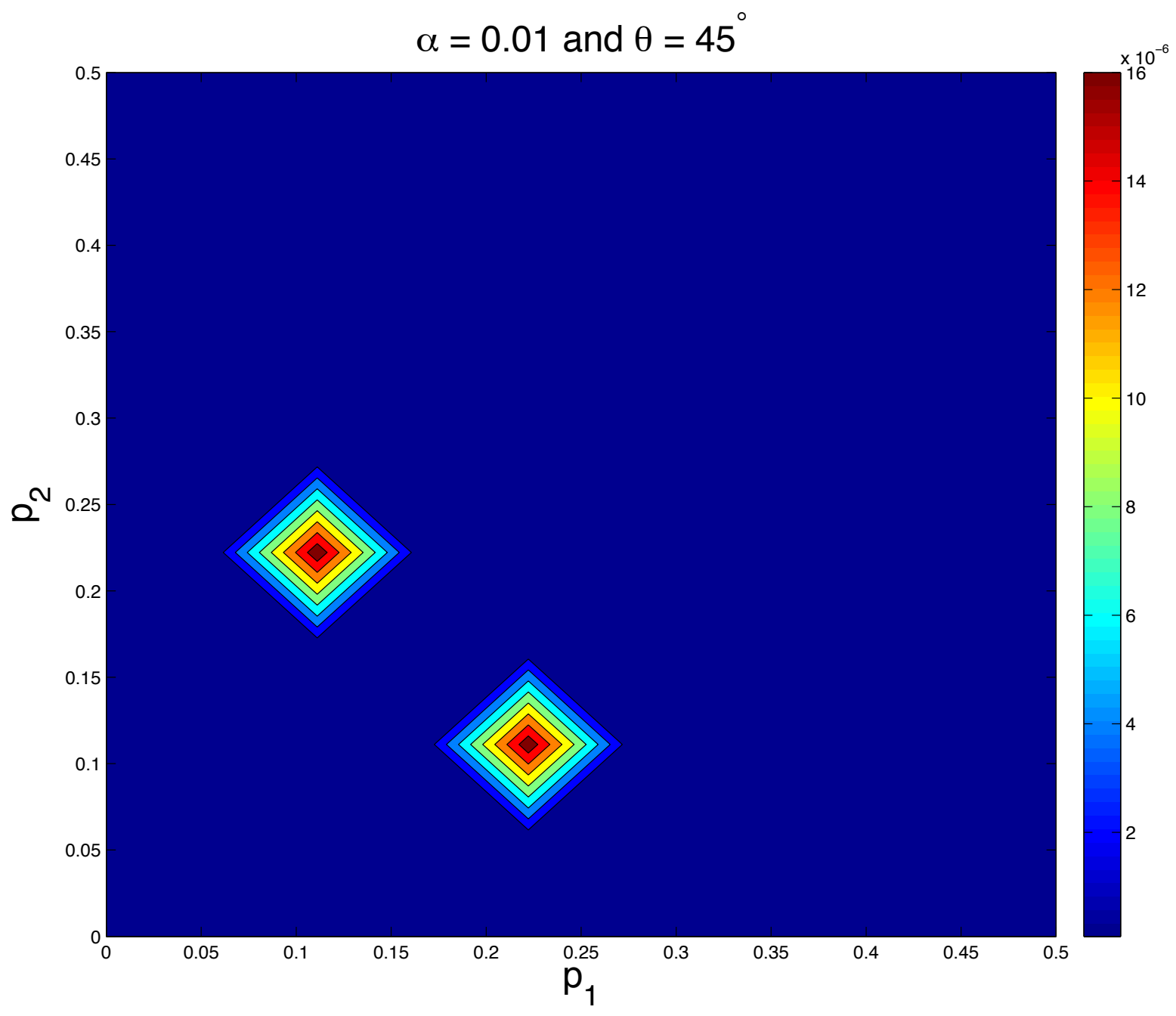

Figure 14. $r_{\max }\left(45^{\circ}, \rho_{1}, \rho_{2} ; 0.010\right)$ versus quasi period $\left(p_{1}, p_{2}\right)$ for $\frac{\rho_{1}}{\rho_{2}}=\frac{1}{4}$. 


\section{4 $\theta=60^{\circ}$ : Moderately Skewed}

In Figures 15 through 17, we see stability behavior that is quite different from the $\theta=45^{\circ}$ case. There are no Zones of Instability. Instead, there are critical values of $\alpha$, displayed in Table IV, beyond which all waves of greater amplitude are unstable. As with $\theta=45^{\circ}$, the onset of instability occurs for smaller values of $\alpha$ as $\frac{\rho_{1}}{\rho_{2}}$ decreases.

\begin{tabular}{|c|c|}
\hline$\frac{\rho_{1}}{\rho_{2}}$ & Critical value of $\alpha$ \\
\hline \hline 1 & 0.015 \\
\hline$\frac{1}{2}$ & 0.013 \\
\hline$\frac{1}{4}$ & 0.0125 \\
\hline
\end{tabular}

TABLE IV

CRITICAL VALUES OF $\alpha$ FOR $\theta=60^{\circ}$. 


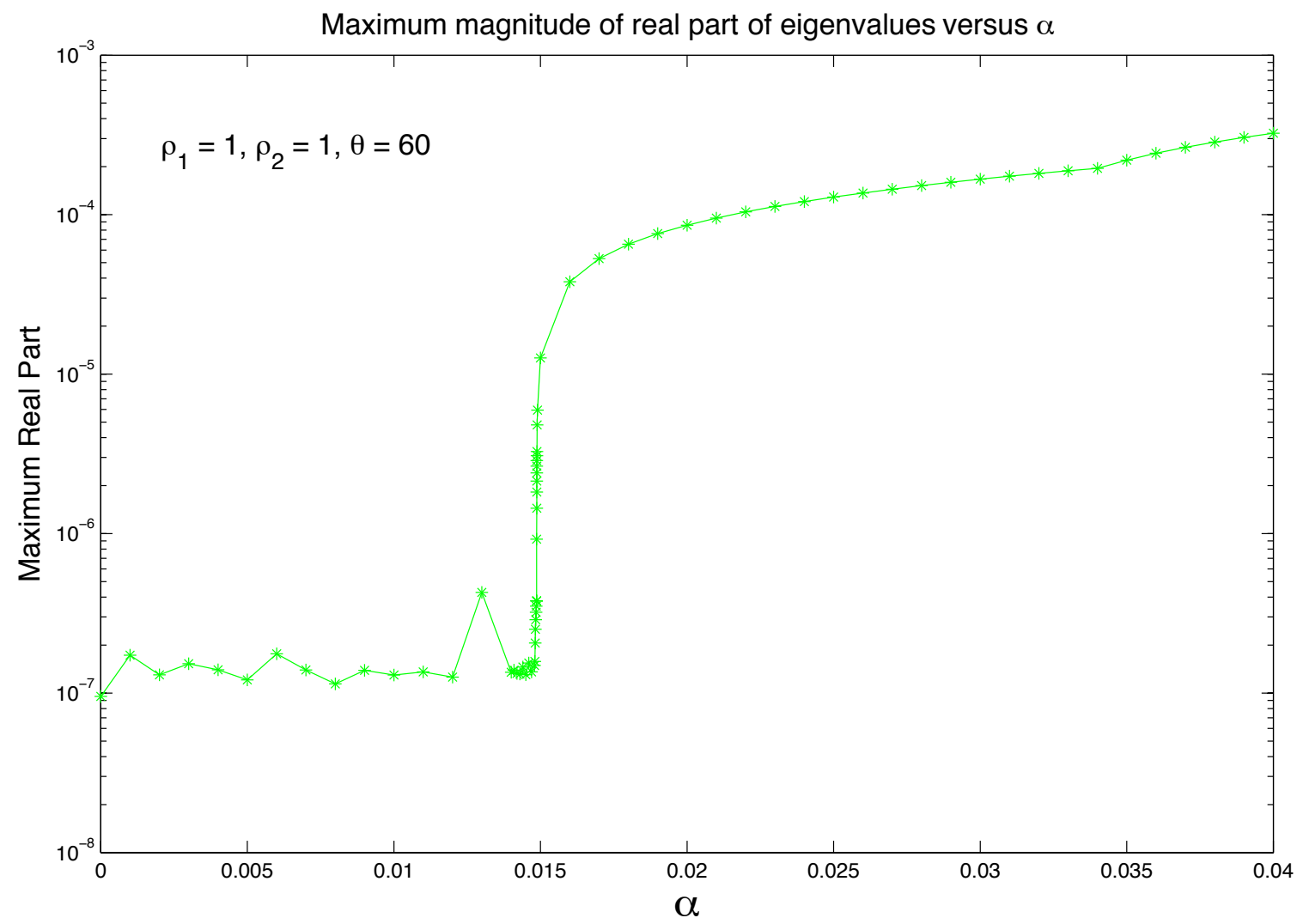

Figure 15. $r_{\max }\left(60^{\circ}, \rho_{1}, \rho_{2} ; \alpha\right)$ versus wave height/slope $\alpha$ for $\frac{\rho_{1}}{\rho_{2}}=1$. 


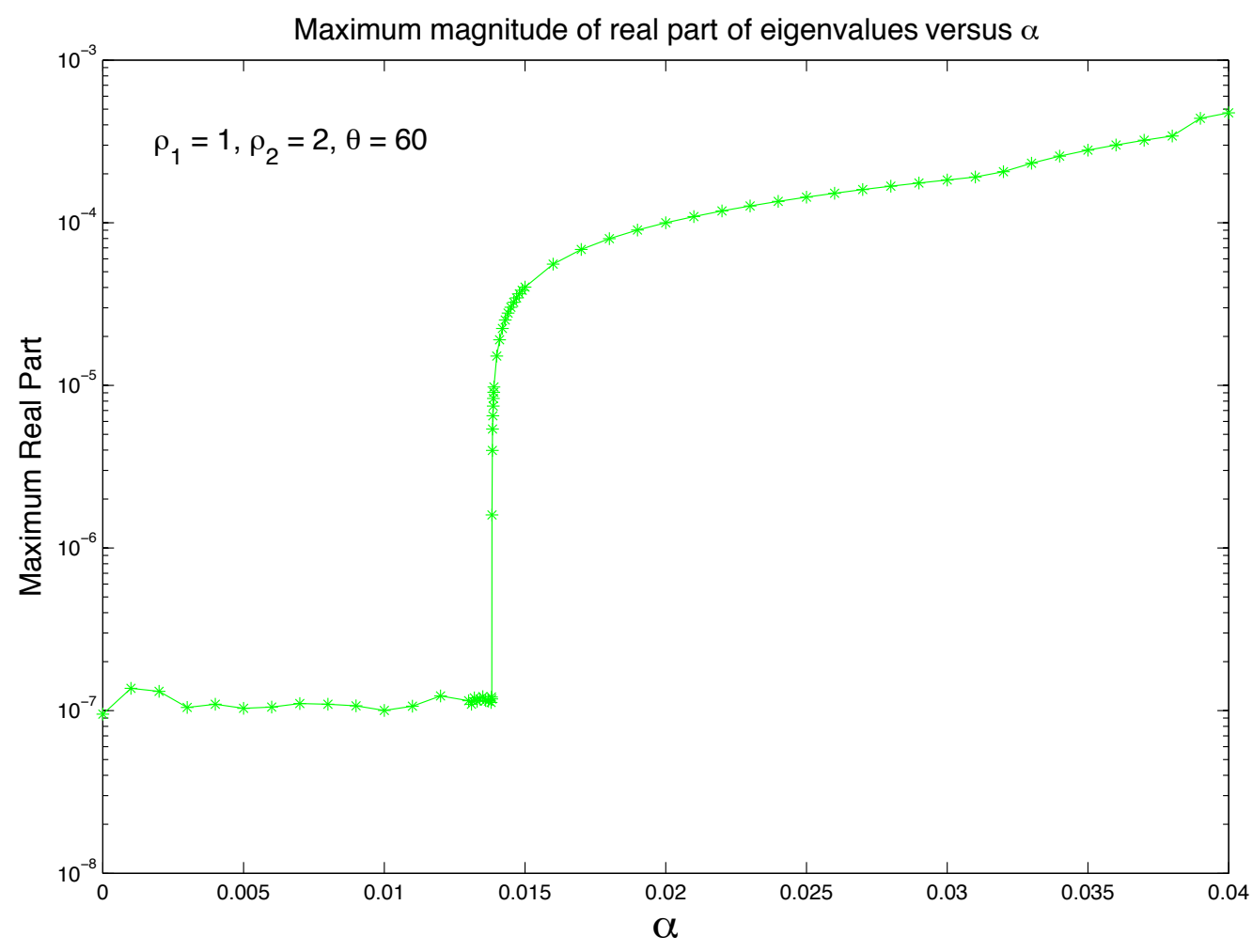

Figure 16. $r_{\max }\left(60^{\circ}, \rho_{1}, \rho_{2} ; \alpha\right)$ versus wave height/slope $\alpha$ for $\frac{\rho_{1}}{\rho_{2}}=\frac{1}{2}$. 


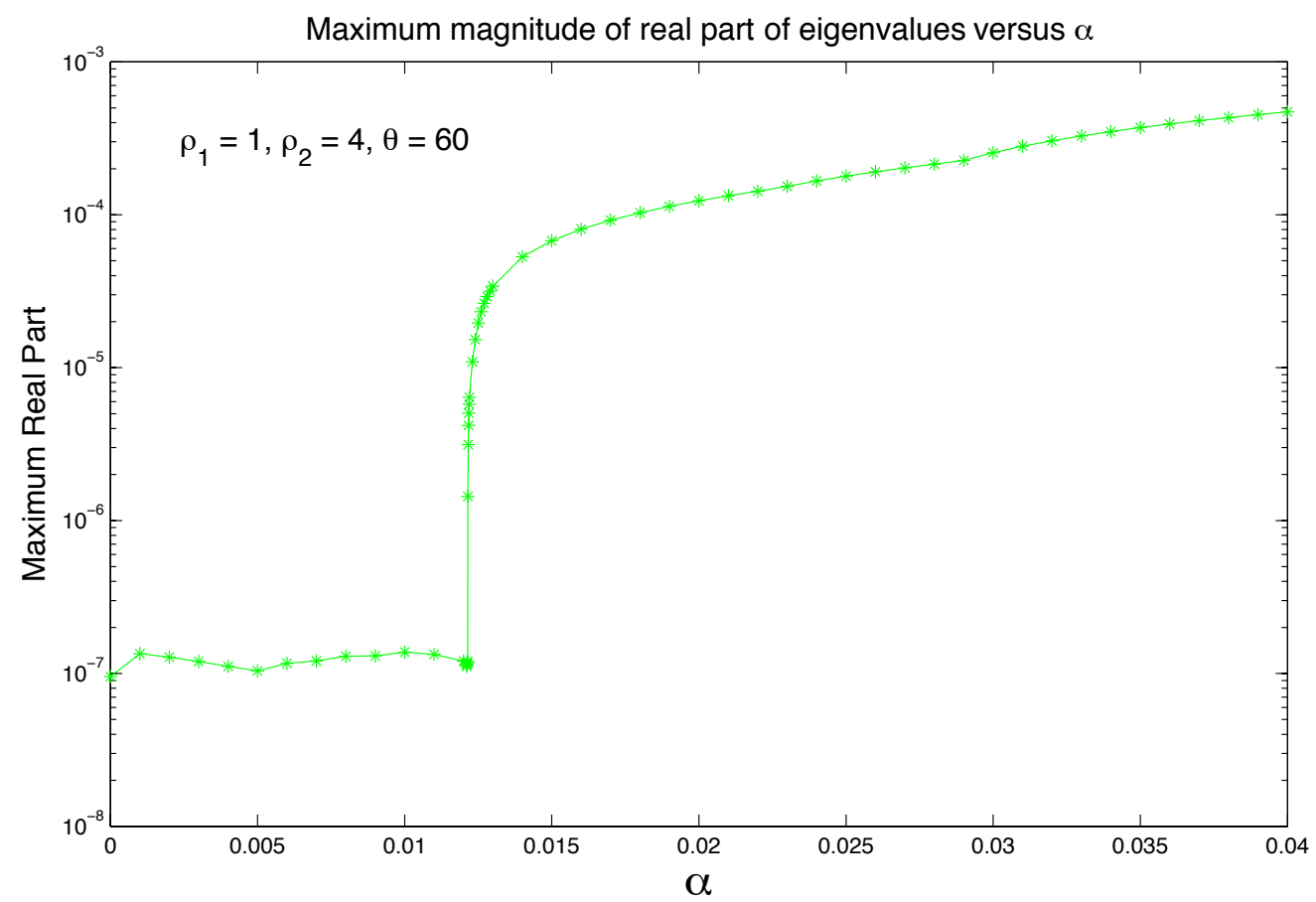

Figure 17. $r_{\max }\left(60^{\circ}, \rho_{1}, \rho_{2} ; \alpha\right)$ versus wave height/slope $\alpha$ for $\frac{\rho_{1}}{\rho_{2}}=\frac{1}{4}$.

In Figures 18 through 20, we display plots of $r_{\max }\left(60^{\circ}, \rho_{1}, \rho_{2}, \alpha\right)$ versus $\left(p_{1}, p_{2}\right)$ to illustrate where the dominant instability occurs with respect to the quasiperiod for a chosen value of $\alpha$. See Table V below. 


\begin{tabular}{|c|c|c|c|c|}
\hline$\frac{\rho_{1}}{\rho_{2}}$ & $\alpha$ & $p_{1}$ & $p_{2}$ & Max Level \\
\hline \hline 1 & 0.020 & 0.0555556 & 0.5 & $8.596479720424639 \times 10^{-5}$ \\
\hline$\frac{1}{2}$ & 0.020 & 0.0555556 & 0.5 & $9.986534811635988 \times 10^{-5}$ \\
\hline$\frac{1}{4}$ & 0.020 & 0.0555556 & 0 & 0.0001234101514083296 \\
\hline
\end{tabular}

TABLE V

DOMINANT INSTABILITY FOR A CHOSEN VALUE OF $\alpha$ FOR $\theta=60^{\circ}$. 


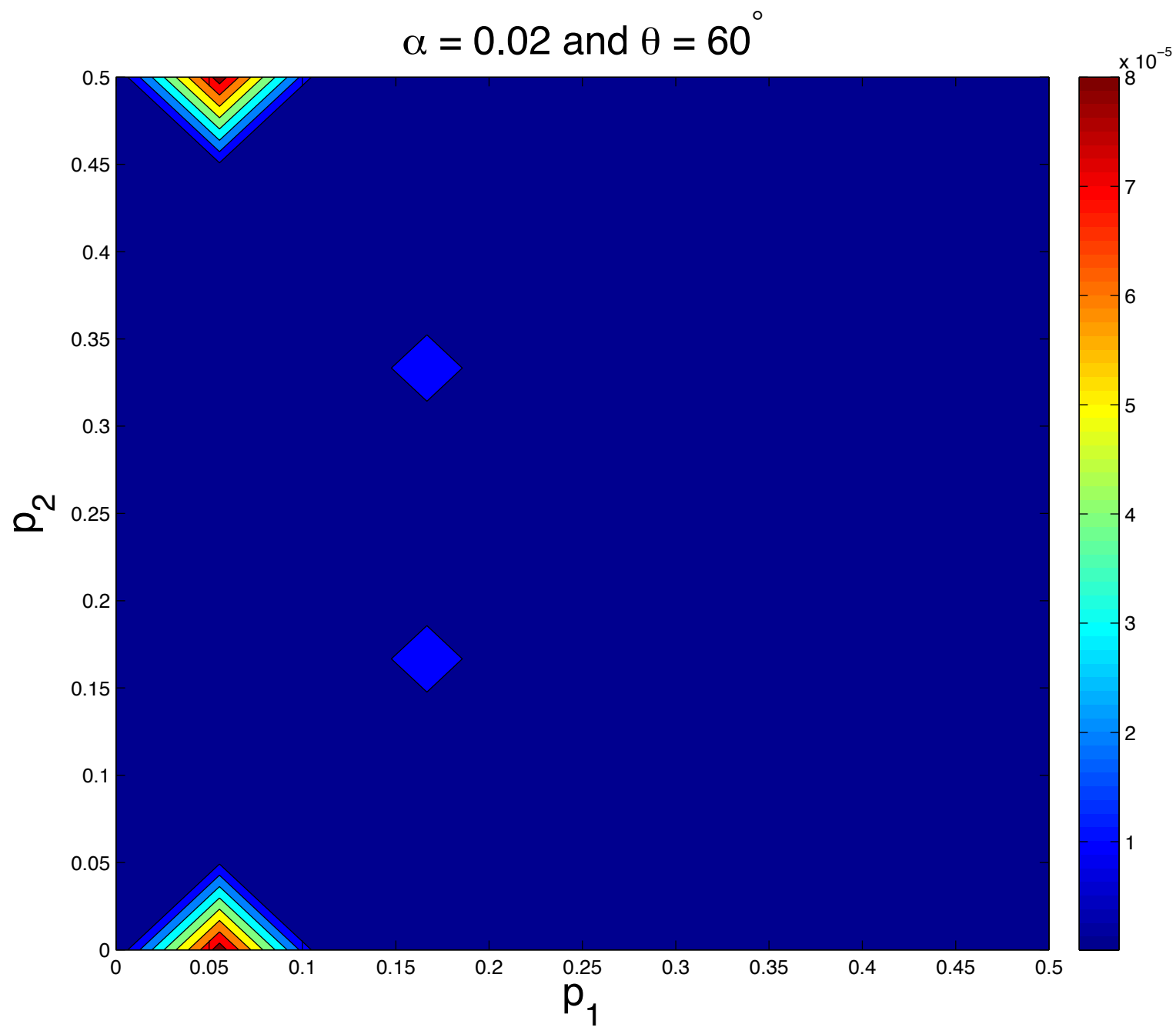

Figure 18. $r_{\max }\left(60^{\circ}, \rho_{1}, \rho_{2} ; 0.020\right)$ versus quasi period $\left(p_{1}, p_{2}\right)$ for $\frac{\rho_{1}}{\rho_{2}}=1$. 


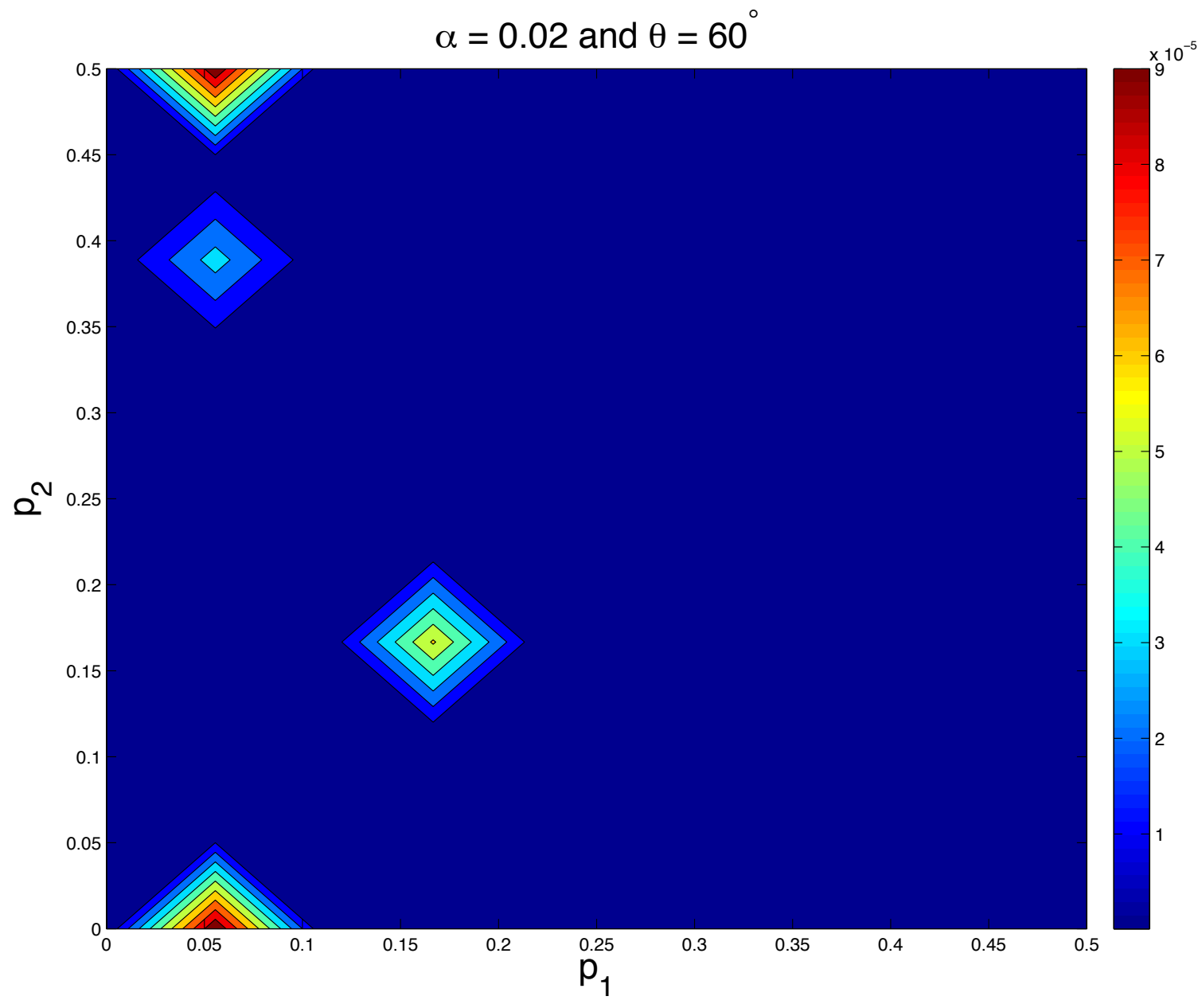

Figure 19. $r_{\max }\left(60^{\circ}, \rho_{1}, \rho_{2} ; 0.020\right)$ versus quasi period $\left(p_{1}, p_{2}\right)$ for $\frac{\rho_{1}}{\rho_{2}}=\frac{1}{2}$. 


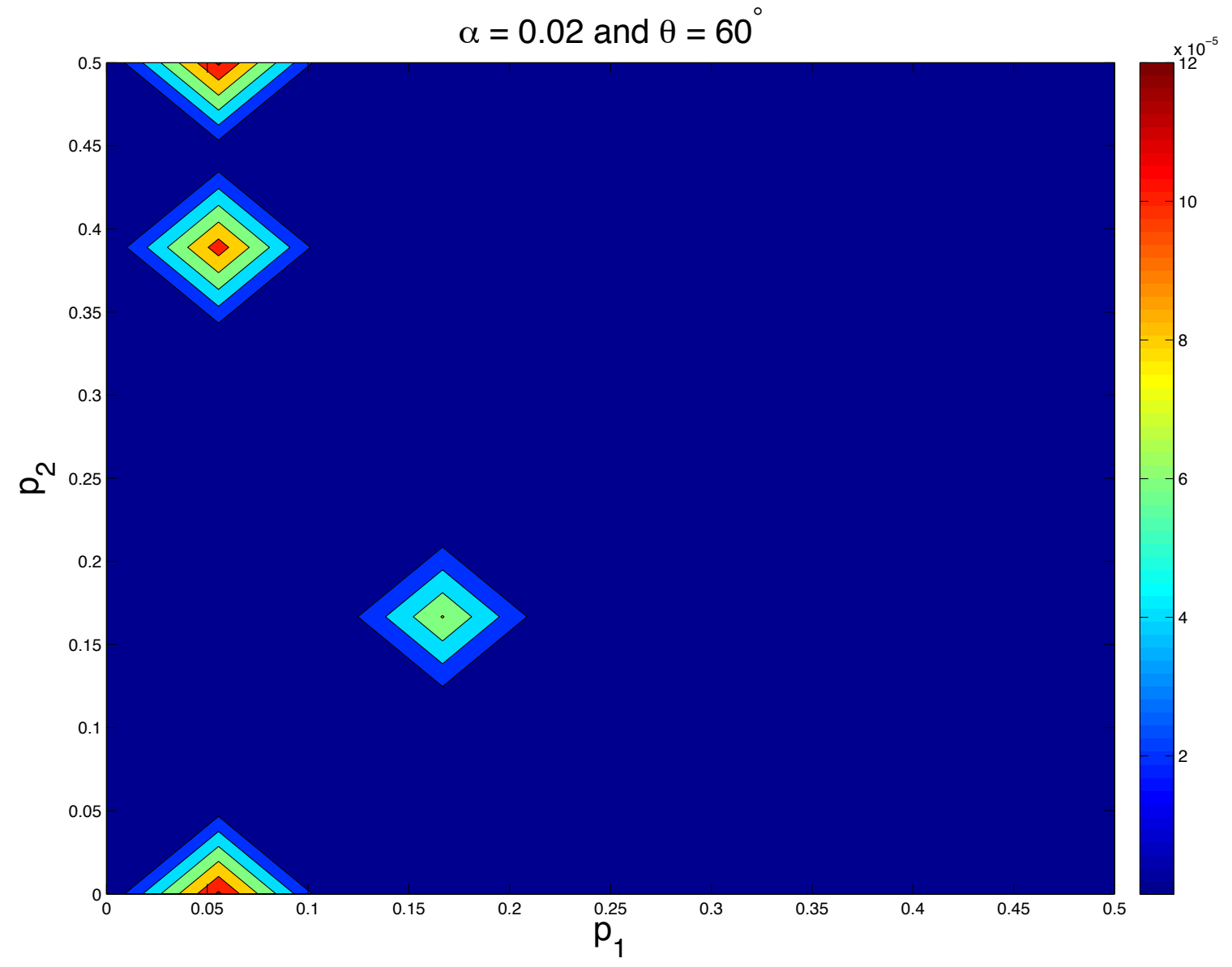

Figure 20. $r_{\max }\left(60^{\circ}, \rho_{1}, \rho_{2} ; 0.020\right)$ versus quasi period $\left(p_{1}, p_{2}\right)$ for $\frac{\rho_{1}}{\rho_{2}}=\frac{1}{4}$. 


\section{5 $\quad \theta=75^{\circ}$ : Strongly Asymmmetric}

In Figures 21 through 23, we again see stability behavior that is quite different from the $\theta=45^{\circ}$ case. There are no Zones of Instability. Instead, as is the case with $\theta=60^{\circ}$, there are critical values of $\alpha$, displayed in Table VI, beyond which all waves of greater amplitude are unstable. As with $\theta=45^{\circ}$ and $\theta=60^{\circ}$, the onset of instability occurs for smaller values of $\alpha$ as $\frac{\rho_{1}}{\rho_{2}}$ decreases. Overall, in examining all of the plots for $\alpha$ versus $r_{\max }$, we deduce a greater degree of instability as $\theta$ is increased from $45^{\circ}$.

\begin{tabular}{|c|c|}
\hline$\frac{\rho_{1}}{\rho_{2}}$ & Critical value of $\alpha$ \\
\hline \hline 1 & 0.012 \\
\hline$\frac{1}{2}$ & 0.008 \\
\hline$\frac{1}{4}$ & 0.0075 \\
\hline
\end{tabular}

TABLE VI

CRITICAL VALUES OF $\alpha$ FOR $\theta=75^{\circ}$. 


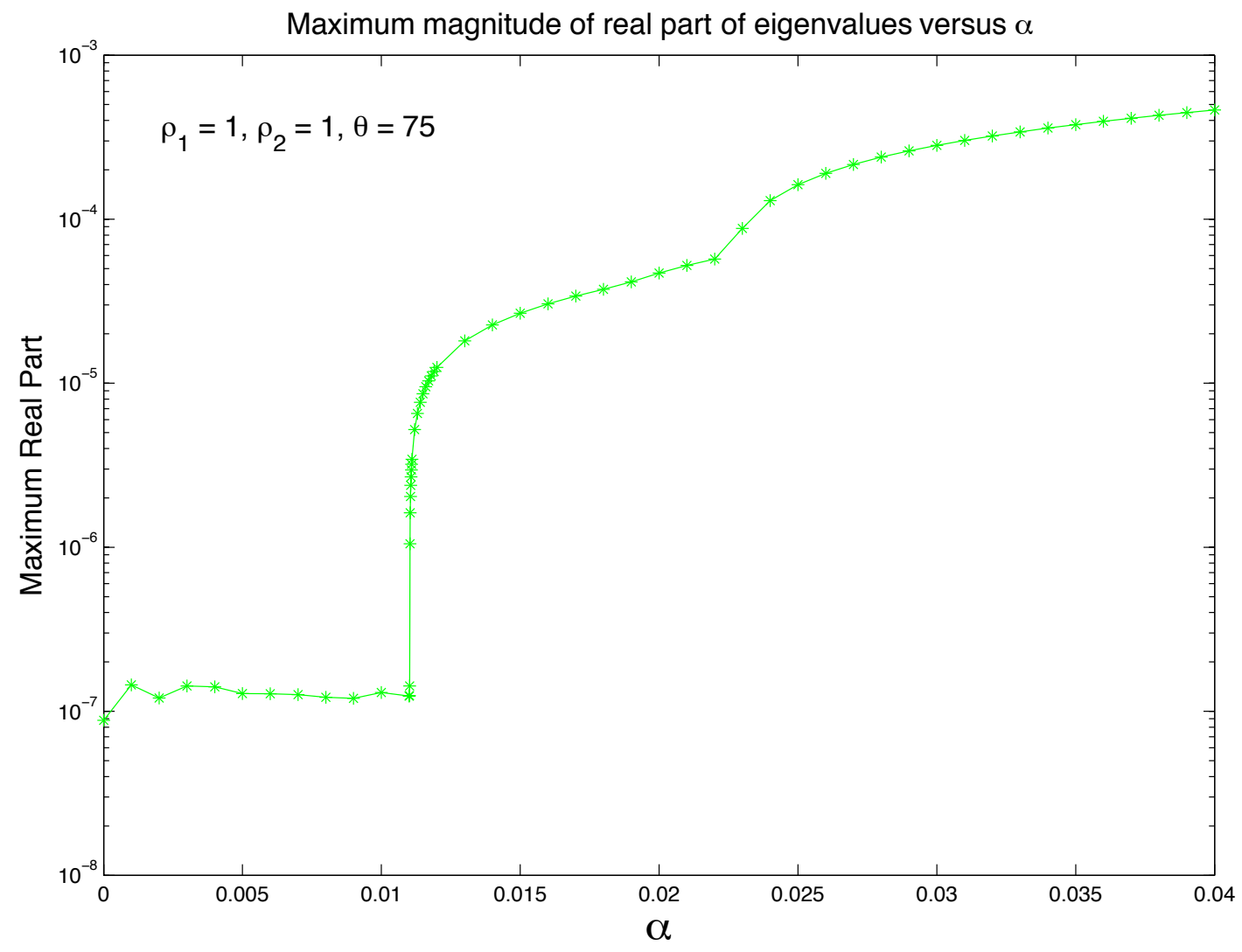

Figure 21. $r_{\max }\left(75^{\circ}, \rho_{1}, \rho_{2} ; \alpha\right)$ versus wave height/slope $\alpha$ for $\frac{\rho_{1}}{\rho_{2}}=1$. 


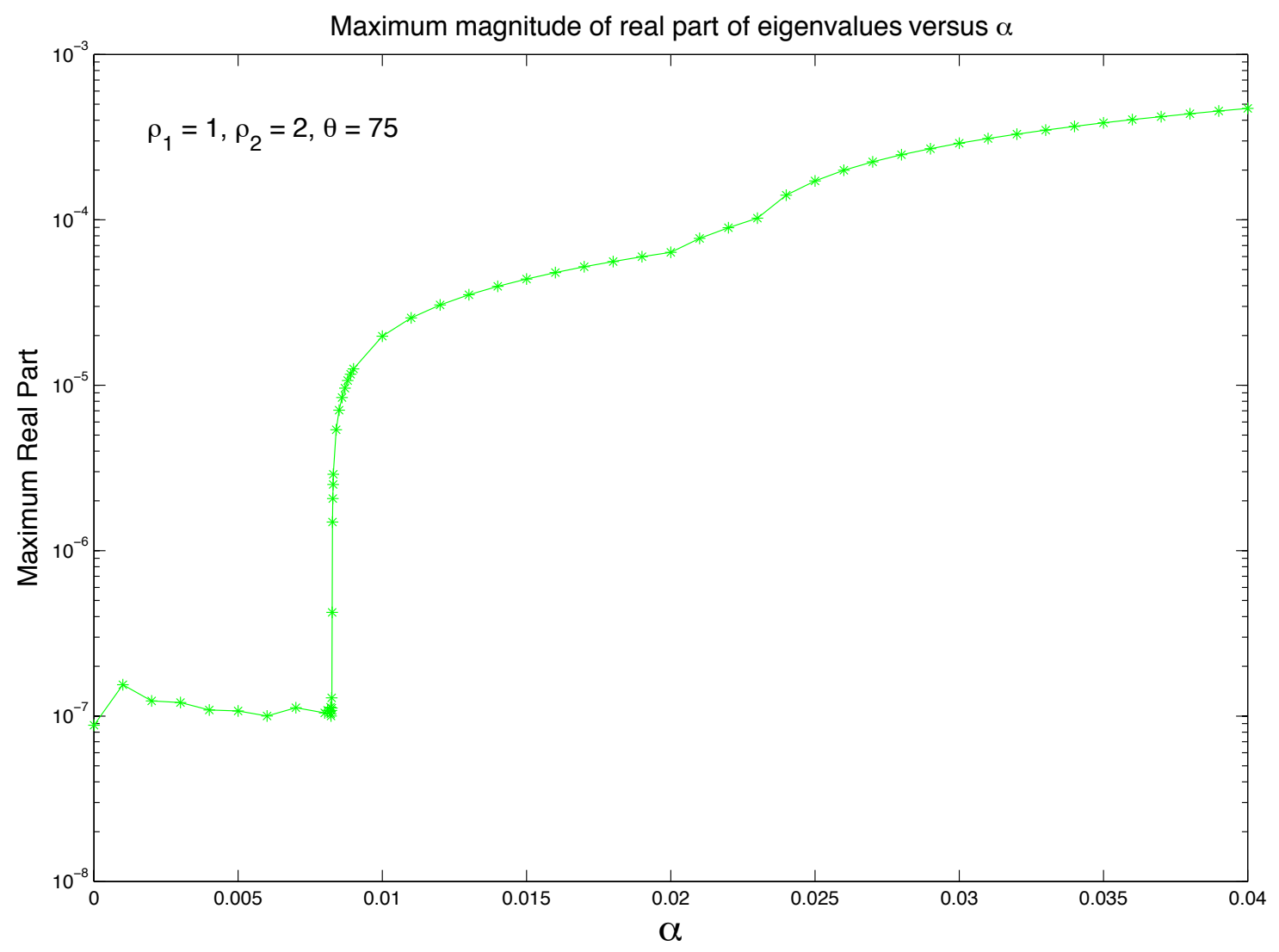

Figure 22. $r_{\max }\left(75^{\circ}, \rho_{1}, \rho_{2} ; \alpha\right)$ versus wave height/slope $\alpha$ for $\frac{\rho_{1}}{\rho_{2}}=\frac{1}{2}$. 


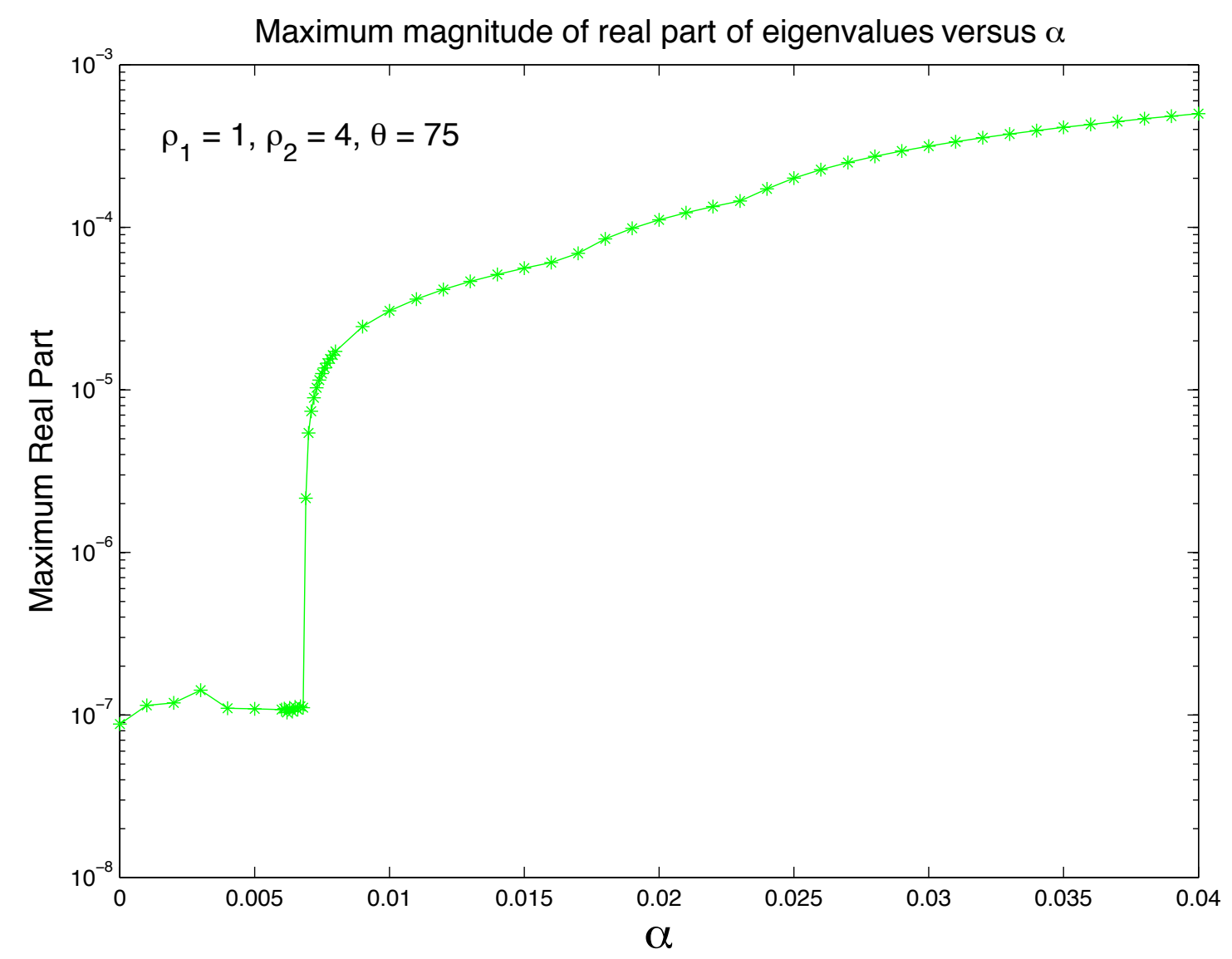

Figure 23. $r_{\max }\left(75^{\circ}, \rho_{1}, \rho_{2} ; \alpha\right)$ versus wave height/slope $\alpha$ for $\frac{\rho_{1}}{\rho_{2}}=\frac{1}{4}$. 
In Figures 24 through 26, we display plots of $r_{\max }\left(75^{\circ}, \rho_{1}, \rho_{2}, \alpha\right)$ versus $\left(p_{1}, p_{2}\right)$ to illustrate where the dominant instability occurs with respect to the quasiperiod for a chosen value of $\alpha$. See Table VII below.

\begin{tabular}{|c|c|c|c|c|}
\hline$\frac{\rho_{1}}{\rho_{2}}$ & $\alpha$ & $p_{1}$ & $p_{2}$ & Max Level \\
\hline \hline 1 & 0.015 & 0.0555556 & 0.277778 & $2.672937205848093 \times 10^{-5}$ \\
\hline$\frac{1}{2}$ & 0.015 & 0.0555556 & 0.277778 & $4.389679638458264 \times 10^{-5}$ \\
\hline$\frac{1}{4}$ & 0.015 & 0.0555556 & 0.277778 & $5.603078904439473 \times 10^{-5}$ \\
\hline
\end{tabular}

TABLE VII

DOMINANT INSTABILITY FOR A CHOSEN VALUE OF $\alpha$ FOR $\theta=75^{\circ}$. 


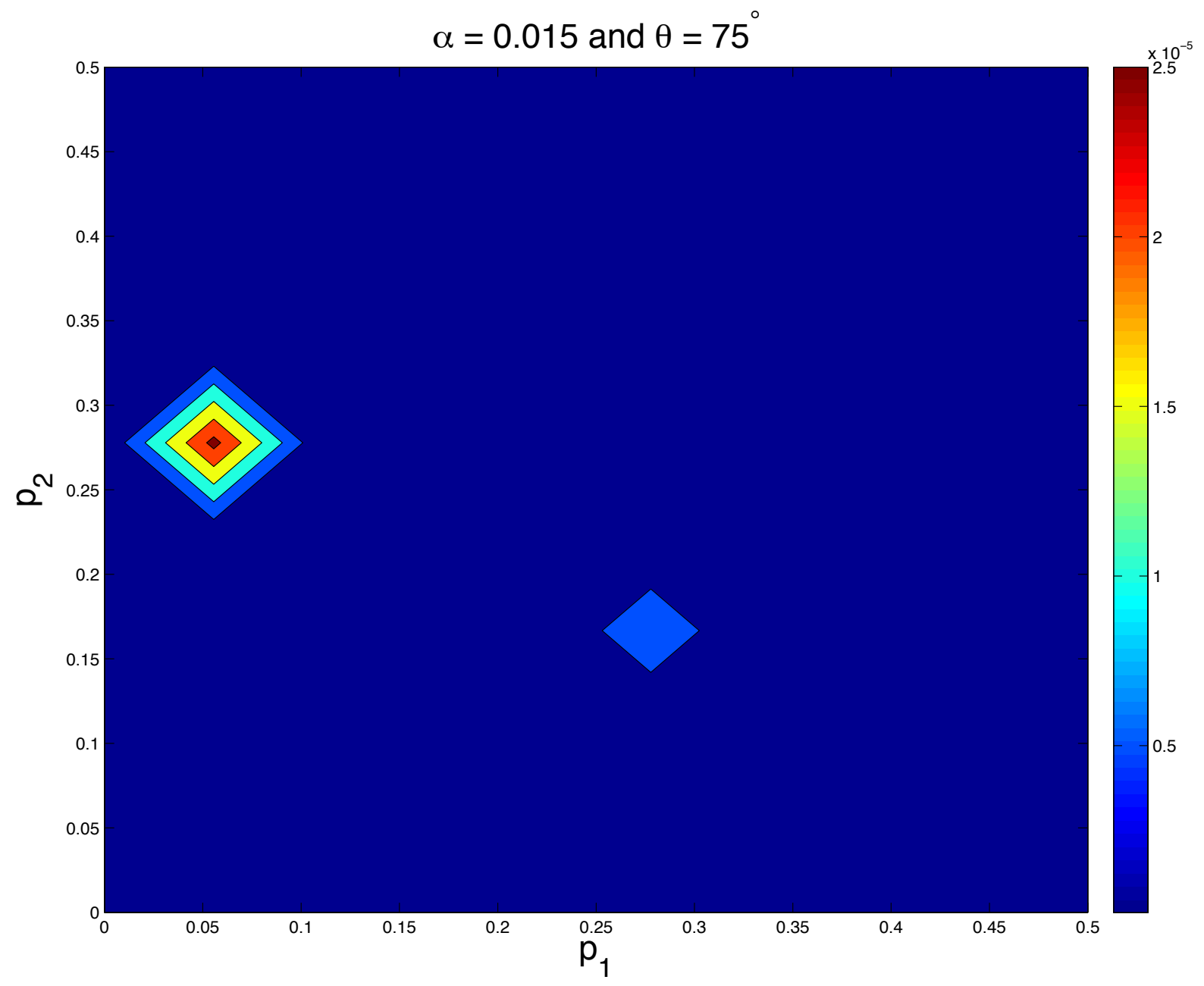

Figure 24. $r_{\max }\left(75^{\circ}, \rho_{1}, \rho_{2} ; 0.015\right)$ versus quasi period $\left(p_{1}, p_{2}\right)$ for $\frac{\rho_{1}}{\rho_{2}}=1$. 


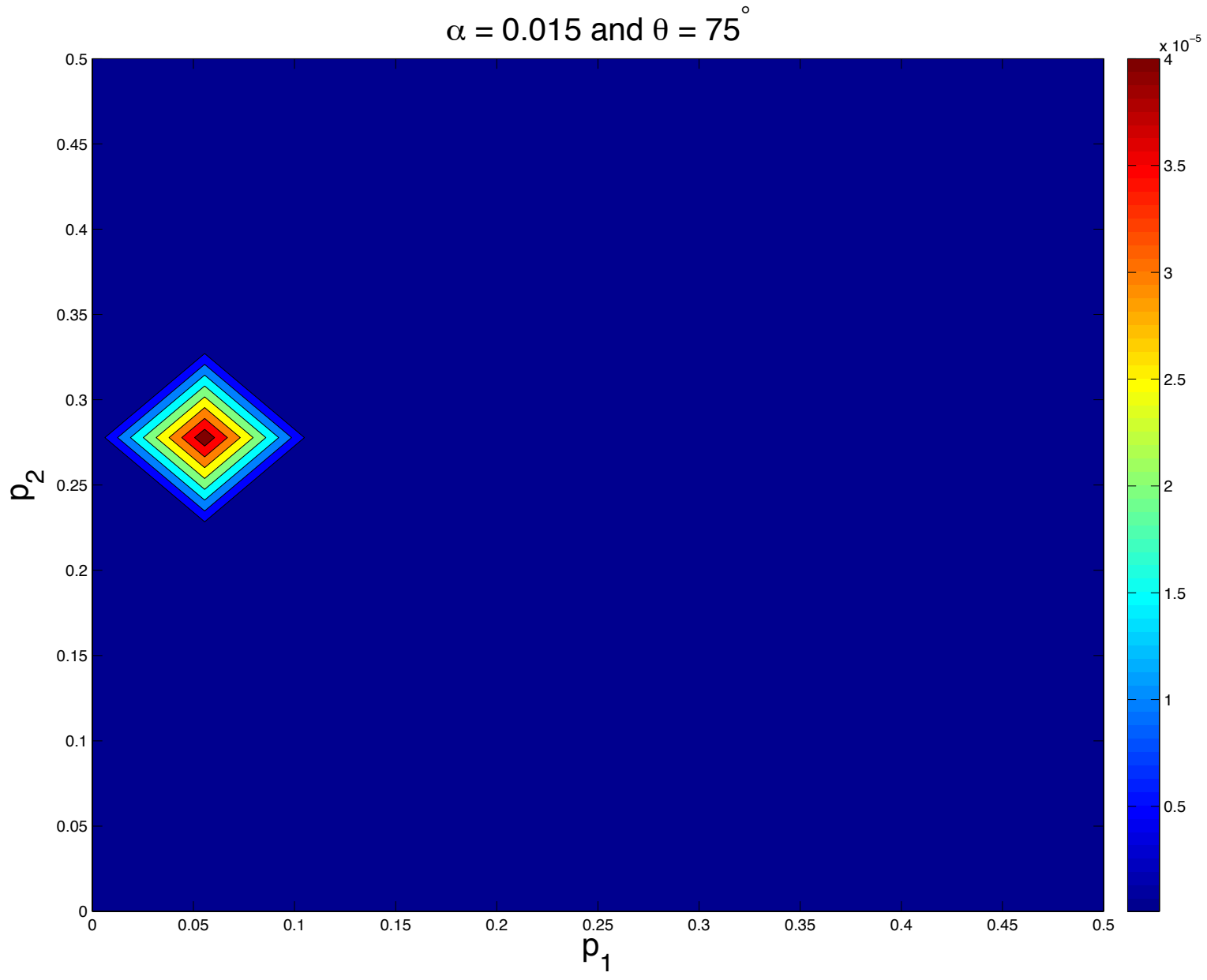

Figure 25. $r_{\max }\left(75^{\circ}, \rho_{1}, \rho_{2} ; 0.015\right)$ versus quasi period $\left(p_{1}, p_{2}\right)$ for $\frac{\rho_{1}}{\rho_{2}}=\frac{1}{2}$. 


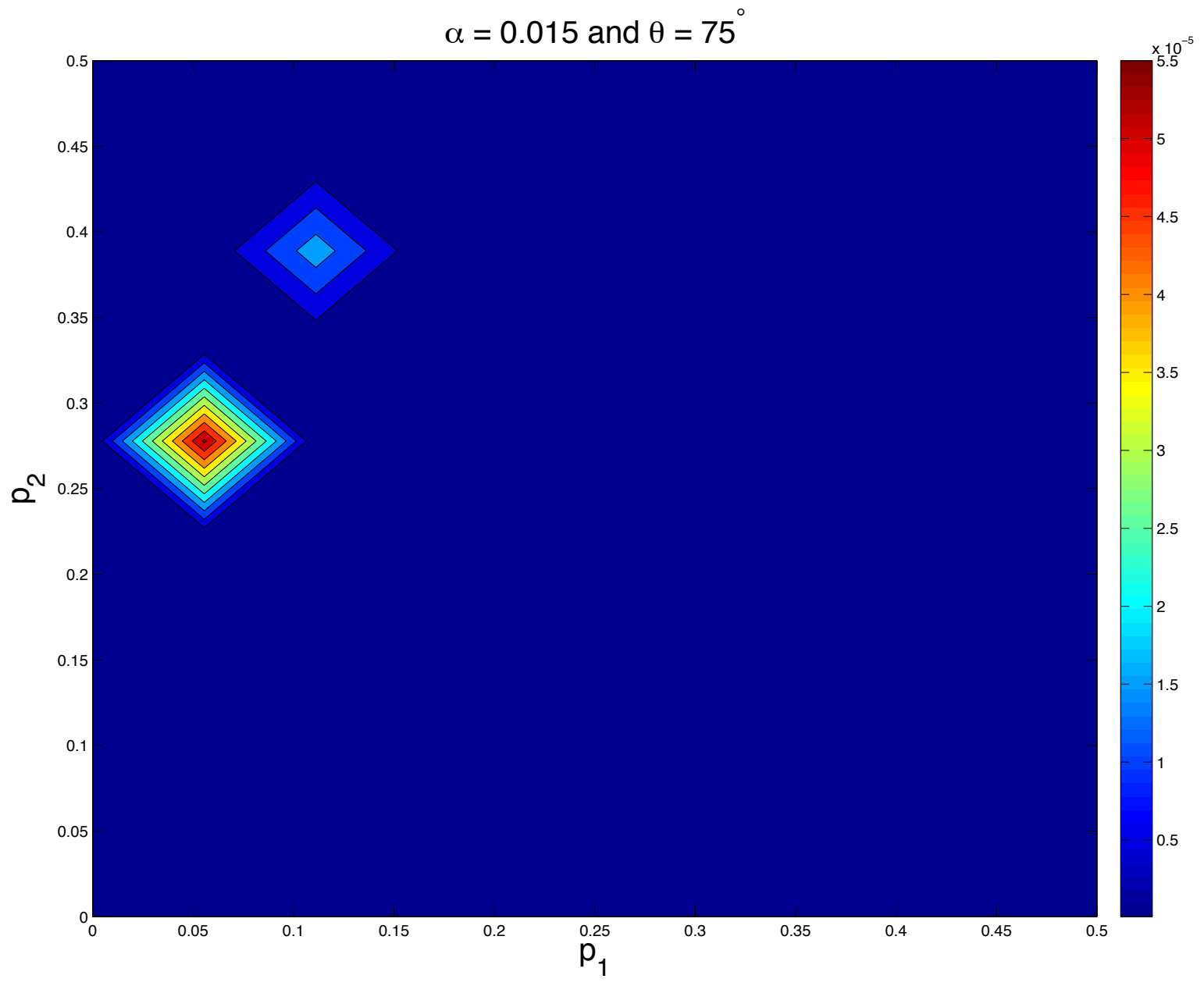

Figure 26. $r_{\max }\left(75^{\circ}, \rho_{1}, \rho_{2} ; 0.015\right)$ versus quasi period $\left(p_{1}, p_{2}\right)$ for $\frac{\rho_{1}}{\rho_{2}}=\frac{1}{4}$. 


\section{CHAPTER 8}

\section{CONCLUSION}

There is more work that can be done for the numerical simulation of GSCWs. For example, with more computing resources, finer meshes of coordinates could be explored for the traveling wave forms as well as the quasi periods. The number of iterations for the DNO and its first variation could also be increased. By exploring a finer mesh of quasi periods, the issue of stability versus instability could be resolved for a greater number of points.

What we have demonstrated within our resources, however, is surprising instability behavior even for the case of a symmetric underlying geometry for a classical SCW $\left(\frac{\rho_{1}}{\rho_{2}}=1\right.$ and $\left.\theta=45^{\circ}\right)$. Overall, for GSCWs we have found stable traveling waveforms which eventually destabilize, with features that depend strongly on the problem's configuration. We saw that within a given geometrical configuration, as a GSCW leading-order coefficient ratio is varied, these waves become more unstable as they become more asymmetric.

Our conclusions about stability for GSCWs were also driven by the spectral data of the resulting linear operator as was that of the investigations into SCWs in the existing literature, but our focus on the role of asymmetry in terms of their geometric configuration via their underlying period, as well as their linear character through the leading-order coefficient ratio $\frac{\rho_{1}}{\rho_{2}}$, sheds new light on the stability behavior of a broader class of short-crested waves. 
APPENDICES 


\section{Appendix A}

\section{PROPERTIES OF THE DNO}

Given Definition 2.2.1, we have the following properties of the DNO:

1. Given $\beta \in \mathbf{R}, G(\eta)[\beta \xi]=\beta G(\eta)[\xi]$.

$\beta \xi$ has potential $\beta \varphi$, thus

$$
\begin{aligned}
G(\eta)[\beta \xi] & =-\left.\nabla_{x} \eta \cdot \nabla_{x}(\beta \varphi)\right|_{y=\eta}+\left.\partial_{y}(\beta \varphi)\right|_{y=\eta} \\
& =\beta\left(-\left.\nabla_{x} \eta \cdot \nabla_{x} \varphi\right|_{y=\eta}+\left.\partial_{y} \varphi\right|_{y=\eta}\right) \\
& =\beta G(\eta)[\xi] .
\end{aligned}
$$

2. $G(\eta)[\xi+\psi]=G(\eta)[\xi]+G(\eta)[\psi]$.

If $\psi=\varphi^{\prime}(x, \eta(x, t), t)$, then

$$
\begin{aligned}
G(\eta)[\xi+\psi] & =-\left.\nabla_{x} \eta \cdot \nabla_{x}\left(\varphi+\varphi^{\prime}\right)\right|_{y=\eta}+\left.\partial_{y}\left(\varphi+\varphi^{\prime}\right)\right|_{y=\eta} \\
& =-\left.\nabla_{x} \eta \cdot \nabla_{x} \varphi\right|_{y=\eta}+\left.\partial_{y} \varphi\right|_{y=\eta}+-\left.\nabla_{x} \eta \cdot \nabla_{x} \varphi^{\prime}\right|_{y=\eta}+\left.\partial_{y} \varphi^{\prime}\right|_{y=\eta} \\
& =G(\eta)[\xi]+G(\eta)[\psi] .
\end{aligned}
$$

3. $G(\eta+\gamma)[\xi] \neq G(\eta)[\xi]+G(\gamma)[\xi]$.

This is clear from the definition of the DNO. 


\section{Appendix A (Continued)}

4. $G(\eta)[f(t) \cdot \xi]=f(t) \cdot G(\eta)[\xi]$.

$$
\begin{aligned}
G(\eta)[f(t) \cdot \xi] & =-\left.\nabla_{x} \eta \cdot \nabla_{x}(f(t) \cdot \varphi)\right|_{y=\eta}+\left.\partial_{y}(f(t) \cdot \varphi)\right|_{y=\eta} \\
& =f(t) \cdot\left(-\left.\nabla_{x} \eta \cdot \nabla_{x} \varphi\right|_{y=\eta}+\left.\partial_{y} \varphi\right|_{y=\eta}\right) \\
& =f(t) \cdot G(\eta)[\xi] .
\end{aligned}
$$

5. Given $\beta \in \mathbf{R}, G_{n}(\eta)[\beta \xi]=\beta G_{n}(\eta)[\xi]$ for all $n \geq 0$.

Let $f=\eta$.

For $n=0$, using (Equation 3.0.3) we have

$$
G_{0}(\eta)[\beta \xi]=|D|[\beta \xi]=\beta|D|[\xi]=\beta G_{0}(\eta)[\xi] .
$$

For $n \geq 1$, using (Equation 3.0.5), we have

$$
\begin{aligned}
G_{n}(\eta)[\beta \xi] & =D\left[F_{n}|D|^{n-1} D[\beta \xi]\right]-\sum_{m=0}^{n-1} G_{m}(\eta)\left[F_{n-m}|D|^{n-m}[\beta \xi]\right] \\
& =\beta\left(D\left[F_{n}|D|^{n-1} D[\xi]\right]-\sum_{m=0}^{n-1} G_{m}(\eta)\left[F_{n-m}|D|^{n-m}[\xi]\right]\right) \\
& =\beta G_{n}(\eta)[\xi] .
\end{aligned}
$$

6. From the definition of the DNO, we have

$$
\lim _{\epsilon \rightarrow 0} G(\eta+\epsilon v)[\xi]=G(\eta)[\xi]
$$




\section{CITED LITERATURE}

1. Acheson, D.: Elementary Fluid Dynamics. New York, NY, Oxford University Press, 1998.

2. Craig, W.: Surface water waves and tsunamis. Journal of Dynamics and Differential Equations, 18(3):525-549, 2006.

3. R.Gisler, G.: Tsunami simulations. Annual Review of Fluid Mechanics, 40:71-90, 2008.

4. Olver, P. J.: Introduction to Partial Differential Equations (ebook). Minneapolis, MN, University of Minnesota, 2010.

5. Zrate, A. R. D., Vigo, D. G. A., Nachbin, A., and Choi, W.: A higher-order internal wave model accounting for large bathymetric variations. Studies in Applied Mathematics, 122(3):275-294, April 2009.

6. Johnson, R.: A Modern Introduction to the Mathematical Theory of Water Waves. Cambridge, UK, Cambridge University Press, 2004.

7. Lamb, H.: Hydrodynamics. Cambridge, Cambridge University Press, 6th edition, 1993.

8. Roberts, A.: Highly nonlinear short-crested water waves. Journal of Fluid Mechanics, 135:301-321, 1983.

9. Roberts, A. and Peregrine, D.: Notes on long-crested water waves. Journal of Fluid

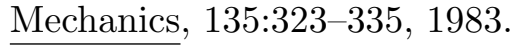

10. Craig, W. and Nicholls, D. P.: Traveling two and three dimensional capillary gravity water waves. SIAM Journal on Mathematical Analysis, 32(2):323-359, 2000.

11. Craig, W. and Nicholls, D. P.: Traveling gravity water waves in two and three dimensions. European Journal of Mechanics B Fluids, 21(6):615-641, 2002.

12. Nicholls, D. P.: Traveling water waves: Spectral continuation methods with parallel implementation. Journal of Computational Physics, 143(1):224-240, 1998. 
13. Nicholls, D. P. and Reitich, F.: Stable, high-order computation of traveling water waves in three dimensions. European Journal of Mechanics B Fluids, 25(4):406-424, 2006.

14. Nicholls, D. P. and Reitich, F.: On analyticity of traveling water waves. Proceedings of the Royal Society of London A, 461(2057):1283-1309, 2005.

15. Ioualalen, M. and Kharif, C.: Stability of three-dimensional progressive gravity waves on deep water to superharmonic disturbances. European Journal of Mechanics B Fluids, 12(3):401-414, 1993.

16. Ioualalen, M. and Kharif, C.: On the subharmonic instabilities of steady three-dimensional deep water waves. Journal of Fluid Mechanics, 262:265-291, 1994.

17. Francius, M. and Kharif, C.: Three-dimensional instabilities of periodic gravity waves in shallow water. Journal of Fluid Mechanics, 561:417-437, 2006.

18. Ioualalen, M., Roberts, A., and Kharif, C.: On the observability of finite-depth shortcrested water waves. Journal of Fluid Mechanics, 322:1-19, 1996.

19. Ioualalen, M., Kharif, C., and Roberts, A.: Stability regimes of finite depth short-crested water waves. Journal of Physical Oceanography, 29(9):2318-2331, 1999.

20. Ioualalen, M. and Okamura, M.: Structure of the instability associated with harmonic resonance of short-crested waves. Journal of Physical Oceanography, 32(5):1331$1337,2002$.

21. Evans, L. C.: Partial Differential Equations. Providence, RI, American Mathematical Society, 1998.

22. Marchant, T. and Roberts, A.: Properties of short-crested water waves in water of finite depth. Journal of the Australian Mathematical Society Series B - Applied Mathematics, 29(1):103-125, 1987.

23. Roberts, A. and Schwartz, L.: The calculation of nonlinear short-crested gravity waves. Physics of Fluids, 26:2388-2392, 1983.

24. Craig, W. and Sulem, C.: Numerical simulation of gravity waves. Journal of Computational Physics, 108:73-83, 1993. 
25. Zakharov, V.: Stability of periodic waves of finite amplitude on the surface of a deep fluid. Journal of Applied Mechanics and Technical Physics, 9:190-194, 1968.

26. Nicholls, D. P.: Spectral stability of traveling water waves: Eigenvalue collision, singularities, and direct numerical simulation. Physica D, 240(4-5):376-381, 2011.

27. Mielke, A.: Instability and stability of rolls in the Swift-Hohenberg equation. Communications in Mathematical Physics, 189(3):829-853, 1997.

28. Deconick, B. and Kutz, J. N.: Computing spectra of linear operators using the FloquetFourier-Hill method. Journal of Computation Physics, 219(1):296-321, 2006.

29. Reed, M. and Simon, B.: Methods of modern mathematical physics. IV. Analysis of operators. New York, Academic Press [Harcourt Brace Jovanovich Publishers, 1978.

30. Milder, D. M.: An improved formalism for rough-surface scattering of acoustic and electromagnetic waves. Proceedings of SPIE - The International Society for Optical Engineering (San Diego, 1991), 1558:213-221, 1991. Int. Soc. for Optical Engineering, Bellingham, WA, 1991.

31. Nicholls, D. P. and Reitich, F.: Analytic continuation of Dirichlet-Neumann operators. Numerische Mathematik, 94(1):107-146, 2003.

32. Nicholls, D. P. and Reitich, F.: A new approach to analyticity of Dirichlet-Neumann operators. Proceedings of the Royal Society of Edinburgh: Section A Mathematics, 131(06):1411-1433, 2001.

33. Fazioli, C. and Nicholls, D. P.: Parametric analyticity of functional variations of DirichletNeumann operators. Differential and Integral Equations, 21(5-6):541-574, 2008.

34. Fazioli, C. and Nicholls, D. P.: Stable computation of variations of Dirichlet-Neumann operators. Journal of Computational Physics, 229(3):906-920, 2010.

35. MacKay, R. and Saffman, P.: Stability of water waves. Proceedings of the Royal Society of London A: Mathematical, Physical and Engineering Sciences, 406:115-125, 1986.

36. Nicholls, D. P.: Spectral data for traveling water waves: Singularities and stability. Journal of Fluid Mechanics, 624:339-360, 2009. 
37. McLean, J., Ma, Y., Martin, D., Saffman, P., and Yuen, H.: Three-dimensional instability of finite-amplitude water waves. Physical Review Letters, 46(13):817-820, 1981.

38. McLean, J. W.: Instabilities of finite-amplitude water waves. Journal of Fluid Mechanics, 114:315-330, 1982.

39. McLean, J. W.: Instabilities of finite-amplitude gravity waves on water of finite depth. Journal of Fluid Mechanics, 114:331-341, 1982.

40. Nicholls, D. P. and Reitich, F.: Shape deformations in rough surface scattering: Cancellations, conditioning, and convergence. Journal of the Optical Society of America, A, 21(4):590-605, 2004.

41. Dias, F. and Kharif, C.: Nonlinear gravity and capillary-gravity waves. Annual review of fluid mechanics, 31:301-346, 1999. Annual Reviews, Palo Alto, CA.

42. Grilli, S., Guyenne, P., and Dias, F.: A fully nonlinear model for three-dimensional overturning waves over an arbitrary bottom. International Journal of Numerical Methods in Fluids, 35:829-867, 2001.

43. Rienecker, H. and Fenton, J.: A fourier approximation method for steady water waves. Journal of Fluid Mechanics, 104:119-137, 1981.

44. Canuto, C., Hussani, M., Quarteroni, A., and Zang, T.: $\quad$ Spectral Method In Fluid Dynamics. New York, Springer-Verlag, 1988.

45. Wilkinson, B. and Allen, M.: Parallel Programming: Techniques and Applications Using Networked Workstations and Parallel Computers. Upper Saddle River, New Jersey, Pearson Prentice Hall, 2005.

46. McBride, T. and Nicholls, D.: On stability of generalized short-crested water waves. Physica D, 241(17):1406-1416, September 2012. http://dx.doi.org/10.1016/j.physd.2012.05.009. 


\section{VITA}

\section{Travis R. McBride}

\section{EDUCATION}

B.A., Mathematics, DePaul University, Chicago, IL, 1990.

M.S., Mathematics, University of Illinois at Chicago, Chicago, IL, 1993.

Graduate Courses, DePaul University, School of Computer Science, Chicago, IL, 1995-1997.

Ph.D. Candidate, Mathematics, University of Illinois at Chicago, Chicago, IL, 2006-Present.

\section{PROFESSIONAL EXPERIENCE}

Teaching Assistant, MSCS, University of Illinois at Chicago, Fall 1991.

Statistical Analyst, Kemper National Insurance Companies, Long Grove, IL, 1993-1996.

Applications Developer, Information Resources, Inc., Chicago, IL, 1997-2000.

Technical Analyst and Senior Applications Developer, TransUnion, Chicago, IL, 2000-2006.

Teaching Assistant, MSCS, University of Illinois at Chicago, January 2007 - May 2010.

Research Assistant, MSCS, University of Illinois at Chicago, Summer 2009.

Research Assistant, MSCS, University of Illinois at Chicago, May 2010 - May 2011.

\section{PUBLICATIONS}

T.McBride, D.P.Nicholls, On stability of generalized short-crested water waves, Physica D (2012), doi:10.1016/j.physd.2012.05.009 


\section{MEMBERSHIPS}

American Mathematical Society (AMS)

Society For Industrial and Applied Mathematics

\section{HONORS}

Graduate Student Teaching Award for exceptional teaching, Fall 2008. 